\title{
Developments in \\ Femtosecond Nanoelectronics
}

\author{
Ultrafast Emission and Control \\ of Electrons in Optical Near-Fields
}

\section{DISSERTATION}

zur Erlangung des mathematisch-naturwissenschaftlichen Doktorgrades

\author{
"Doctor rerum naturalium" \\ der Georg-August-Universität Göttingen \\ im Promotionsprogramm ProPhys \\ der Georg-August University School of Science (GAUSS) \\ Von \\ Herrn Dipl.-Phys. Georg Herink \\ aus Höxter
}

Göttingen, 2014 
Betreuungsausschuss

Prof. Dr. Claus Ropers, IV. Physikalisches Institut

Prof. Dr. Tim Salditt, Institut für Röntgenphysik

$\underline{\text { Mitglieder der Prüfungskommission }}$

Referent: $\quad$ Prof. Dr. Claus Ropers, IV. Physikalisches Institut

Korreferent: $\quad$ Prof. Dr. Tim Salditt, Institut für Röntgenphysik

2. Korreferent: $\quad$ Prof. Dr. Bert Hecht, Universität Würzburg

$\underline{\text { Weitere Mitglieder der Prüfungskommission }}$

Prof. Dr. Jörg Enderlein, III. Physikalisches Institut

Prof. Dr. Wolfram Kollatschny, Institut für Astrophysik

Prof. Dr. Werner Lauterborn, III. Physikalisches Institut

Prof. Dr. Vasily Moshnyaga, I. Physikalisches Institut

Tag der mündlichen Prüfung: 16. Dezember 2014 


\section{Abstract}

Coupling light to metal surfaces can break the diffraction limit and concentrate optical fields at the nanoscale. The studies presented in this cumulative thesis investigate the influence of optical field localization onto strong-field photoemission from metallic nanotips in a spectral range from near- to far-infrared frequencies (1 - 400 Terahertz).

Intense infrared excitation is demonstrated to provide access to the strong-field photoemission regime and to the ponderomotive scaling of the electron kinetic energy. Specifically, at long wavelengths, conditions are reached, in which the quiver amplitude of the photoelectrons can exceed the dimension of the localized driving field. As a result, the light-electron interaction is confined to a fraction of the optical half-cycle, enabling quasiinstantaneous field-driven electron acceleration. A spatial adiabaticity parameter is introduced to classify the experimentally observed transition in the electron energy scaling and to distinguish the oscillatory and the sub-cycle acceleration regimes. The adaptation of a two-step model of strong-field photoemission for near-fields allows for the quantitative numerical analysis of the spatio-temporal dynamics and the reproduction of the experimental findings.

In another set of experiments, employing a two-color streaking scheme with nearinfrared and Terahertz (THz) pulses, the local THz-waveform at the emission site is temporally mapped and the sub-cycle acceleration dynamics are resolved in-phase. Moreover, electron dynamics at the transition from oscillatory to sub-cycle acceleration are studied via streaking experiments and numerical simulations. The findings demonstrate new schemes for the ultrafast manipulation of electron trajectories during their transit in time-varying near-fields. Direct applications arise, e.g., in the compression of electron pulses, and are sketched at the end of this work.

Finally, the high field enhancement at long wavelengths is demonstrated to enable ultrafast cold field emission with THz-radiation. Peaked at high energies, the observed energy spectra reveal the characteristic signatures of emission and acceleration in the sub-cycle regime. Moreover, the nonlinearity of the field emission process is employed to temporally track the picosecond carrier cooling at the nanotip apex after intense near-infrared excitation. The findings indicate reduced cooling-rates due to the nanoscopic heat confinement and establish an optical-pump / field-emission-probe scheme for the local access to carrier-dynamics in nanostructures. 


\section{Contents}

1 Introduction 1

2 Mechanisms of light-induced electron emission 5

2.1 Linear photoemission . . . . . . . . . . . . . . . . . 5

2.2 Multiphoton absorption and the transition to strong-field light-matter in-

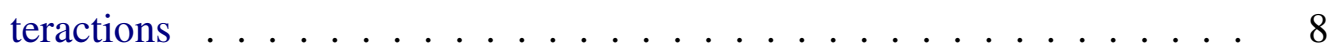

2.3 Strong-field interactions I: Tunnel emission . . . . . . . . . . . . . 10

2.4 Strong-field interactions II: Post-ionization dynamics . . . . . . . . . . . 12

2.5 Strong-field dynamics at nanostructures . . . . . . . . . . . . 15

3 Experimental methods and concepts $\quad 19$

3.1 Generation of femtosecond pulses - ultrafast laser sources . . . . . . . . . 19

3.2 Frequency-tunable pulses via nonlinear optics . . . . . . . . . . . . . 20

3.3 Infrared beam characterization . . . . . . . . . . . . . . . . 22

3.4 Generation of ultrashort Terahertz transients . . . . . . . . . . . . . 25

3.5 Electro-optic sampling . . . . . . . . . . . . . . . . 27

3.6 Photoelectron spectroscopy . . . . . . . . . . . . . . . . . . . . . . 29

3.6.1 Retarding field energy analyzer . . . . . . . . . . . . . . . . 29

3.6.2 Time-of-flight electron spectrometer . . . . . . . . . . . . 30

3.7 Optical setup and experimental chamber . . . . . . . . . . . . . . 32

3.8 Nanoscopically-sharp gold and tungsten needles . . . . . . . . . . . . 33

3.9 Streaking spectroscopy . . . . . . . . . . . . . . . . . . . . 37

3.9.1 All-optical streaking . . . . . . . . . . . . . . . 38

3.9.2 Nanoplasmonic streaking . . . . . . . . . . . . . . . 39

4 Field-driven photoemission from nanostructures quenches the quiver motion 
5 Terahertz control of nanotip photoemission

6 Field emission at Terahertz frequencies: AC-tunneling and ultrafast carrier dynamics in nanotips $\quad 73$

7 Discussion $\quad 83$

7.1 Light-electron interaction in optical near-fields . . . . . . . . . . 83

7.2 THz near-field streak camera . . . . . . . . . . . . . . . . . . 87

7.3 Field emission as an ultrafast probe . . . . . . . . . . . . . . 91

8 Appendix $\quad 93$

8.1 Notations and Abbreviations . . . . . . . . . . . . . . . . 93

8.2 Derivation of linear carrier cooling in the absence of transport . . . . . . 94

$\begin{array}{lr}\text { Bibliography } & 97\end{array}$

$\begin{array}{lr}\text { Publications and conference talks } & 119\end{array}$

$\begin{array}{ll}\text { Danksagung } & 121\end{array}$ 


\section{Chapter 1}

\section{Introduction}

Steering electrons with electric fields forms the basis of today's data processing, X-ray technology, electron microscopy and accelerator science. After the discovery of "cathode rays", electron beams have enabled broad applications - ranging from micro-wave heating and TV-tubes in our homes, radar technology for flight safety, and X-ray sources for computer tomography to ultrahigh-resolution instruments for the lithographic fabrication of nanoscopic circuits and for imaging structures at the atomic level.

However, these instruments are just starting to benefit from the prospects of the precise and dynamic manipulation of electric fields - the realm of modern optics [1, 2]. Ultrafast laser technology provides the highest electric fields and the fastest temporal transients down to attosecond timescales [3, 4]. For the last three decades, the generation and control of electron bunches via ultrashort electric fields has been intensely studied in strong-field ionization of atoms and molecules $[5,6,7]$. The transfer of such concepts to solid nanostructures, however, has recently emerged as a new field of research. Specifically, metallic nanostructures with their intrinsic high carrier densities allow for strong enhancements and sub-wavelength localization of optical fields [8, 9]. Compared to gaseous media, they allow for static biasing, contacting and large scale integration. In addition, the femtosecond-lifetimes of electronic excitations support response frequencies above 50 Terahertz [10].

The combination of tailored laser fields [11] and concepts from ultrafast atomic spectroscopy with the optical properties of metallic nanostructures bears the potential for the localized generation of electron pulses and their control in optical fields. The continued development of these techniques promises to eventually provide ultrafast control of electric currents on the nanoscale. Applications arise in the generation of electron pulses 


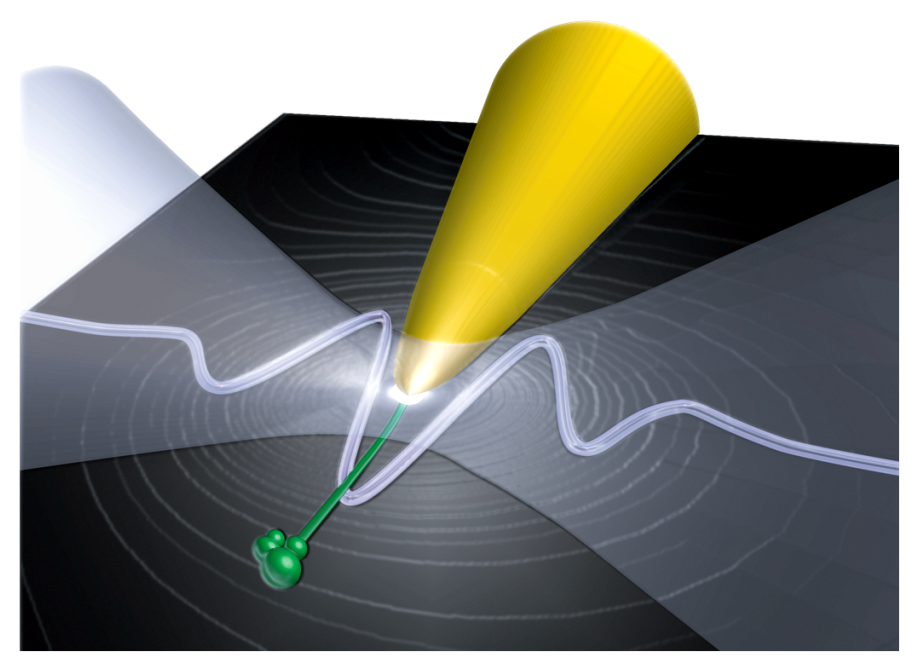

Figure 1.1: Illustration of spatially and temporally confined photoemission from a nanotip.

for ultrafast electron diffraction and microscopy [12, 13, 14], ultrafast X-ray sources $[15,16,17,18]$, and may find uses in future miniaturized high-speed opto-electronic devices. Moreover, the refined understanding of strong-field light-matter interactions at dense targets may be of fundamental interest for the study of ultrafast phenomena in more complex systems, for applications in material processing and medicine [19].

In this thesis, we study ultrafast nonlinear photoemission from metallic nanotips over a spectral range from near-infrared up to Terahertz frequencies. Ultrafast lasers and methods from photoelectron and Terahertz spectroscopy are applied to investigate the impact of the nanostructure near-field confinement on the photoelectron dynamics. Characteristic features of electron acceleration in near-fields are employed in a dual-color pump-probe scheme to map the local waveform of the electric field at the apex. Moreover, the electron dynamics in oscillating near-fields are studied via phase-resolved detection and numerical simulations. Under conditions of intense near-infrared excitation, hot electrons are generated, and the two-color scheme is adapted to temporally track the carrier dynamics locally at the apex.

\section{Outline}

In Chapter 2, different mechanisms of light-induced electron emission are presented in a brief historical context with an emphasis on instantaneous processes. We outline important findings in strong-field atomic ionization and recent experiments on ultrafast photoe- 
mission from metallic nanotips.

In Chapter 3, we introduce the methods for generating ultrafast pulses at various frequencies. Several features of the photoemission apparatus, the characterization of optical transients, and properties of the nanostructures and their preparation are discussed.

In Chapter 4, a photoemission study from gold nanotips at near- and mid-infrared wavelengths up to $8 \mu \mathrm{m}$ is presented. The content of this chapter is published as Ref. [20], "Field-driven photoemission from nanostructures quenches the quiver motion". Here, we demonstrate nonlinear photoemission and electron acceleration above $100 \mathrm{eV}$, deeply accessing the strong-field photoemission regime without sample damage. At long wavelengths, we reach conditions in which the oscillation amplitude of the accelerated electrons exceeds the near-field profile. The electrons can escape the driving field within a fraction of the optical half-cycle, resulting in dynamics that drastically differ from the situation of atomic ionization in macroscopic foci. The results establish a novel interaction regime of strong-field photoemission in optical near-fields.

In Chapter 5, the Terahertz-streaking of photoelectron pulses in the tip-enhanced nearfield is presented. The content of this chapter is published as Ref. [21], "Terahertz control of nanotip photoemission". Single-cycle THz electric transients are employed to control NIR-induced photoemission from gold nanotips in a two-color experiment. The electron acceleration in the $\mathrm{THz}$ near-field allows for a mapping of the local $\mathrm{THz}$ waveform and for a spectral reshaping of photoelectron distributions. Due to the carrier-envelope-phase stability of the THz waveform, the specific sub-cycle acceleration dynamics in the nearfield can be resolved in-phase.

In Chapter 6, the direct field emission via $\mathrm{THz}$ pulses from a tungsten nanotip is presented. The content of this chapter is published as Ref. [22], entitled "Field emission at Terahertz frequencies: AC-tunneling and ultrafast carrier dynamics in nanotips". This first demonstration of field emission at $\mathrm{THz}$ frequencies represents the long wavelength limit of strong-field photoemission, and results in characteristic quasi-instantaneous electron energy distributions. In a local pump-probe scheme, the nonlinear THz-induced tunneling is applied to temporally resolve the picosecond relaxation of NIR-induced hot electrons at the nanotip apex, providing access to ultrafast carrier dynamics inside the nanostructure.

In Chapter 7, we present the main results of the preceding studies and discuss their implications in a broader context. Several concepts for potential applications in science and technology are outlined. 


\section{Chapter 2}

\section{Mechanisms of light-induced electron emission}

The light-induced liberation of electrons from matter is enabled via a diverse set of physical processes. Their discovery often challenged existing theories and promoted our understanding of elementary light-electron interactions.

In this chapter, we review the evolution from linear to nonlinear and strong-field photoemission at high intensities. We focus onto emission processes which preserve the temporal confinement of an ultrashort optical trigger (below $100 \mathrm{fs}$ ) to allow for the generation of ultrashort electron pulses. The development of the physical pictures for lightelectron interactions is sketched in a brief historical context via key experiments. Several observations of strong-field ionization at atoms and molecules are explicitly revisited, as they form the basis of the strong-field picture for photoemission at solid nanostructures. Recent studies on nonlinear and strong-field photoemission from metallic nanotips are presented at the end.

\subsection{Linear photoemission}

The photoeffect was found by Hertz in 1887 [23], ten years before Thomson's actual discovery of the "electron" as the quantum of negative electric charge. Originating in the observation of enhanced arc-discharges under UV-illumination, the light-induced charging of metal plates and generation of cathode-rays were thoroughly studied by Hallwachs [24, 25] and Lenard [26, 27], respectively. Lenard's experimental findings, in particular the intensity-independence of the photoelectron kinetic energy, were in direct contradiction to the classical expectation of the light-electron interaction. According to an electrodynamic picture, the photoelectron kinetic energy grows with longer driving wave- 


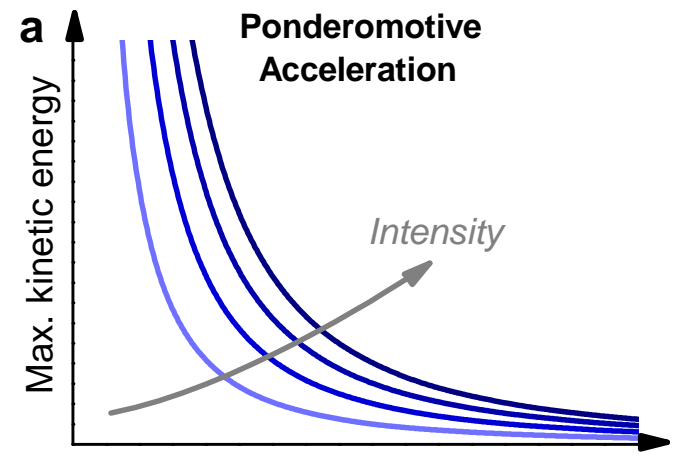

Light frequency $\omega$

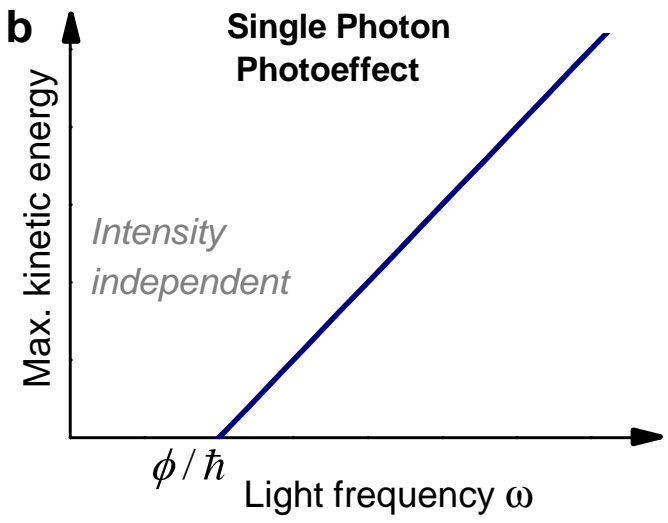

Light frequency $\omega$

Figure 2.1: a) According to classical mechanics, the kinetic energy of a photoemitted electron increases with the intensity and decreases with the frequency of the oscillating electric field proportional to the ponderomotive energy. b) In contrast, Einstein's photoelectric law states the linear increase of the electron energy with the light frequency and the intensity independence, according to experimental observations.

length and is intensity dependent, illustrated in Figure 2.1. A sketch of Lenard's setup in Figure 2.2a reveals the basic components of a typical photoemission spectroscopy experiment. In his perceptive analysis of the experimental findings, he concludes that the detected electron kinetic energies of several $\mathrm{eV}$ are not available from light of usual intensity, and speculates that the electron energy may not be provided by the light at all [27].

A short calculation based on classical mechanics proves his objection and introduces the ponderomotive energy. We consider the motion of an electron in an oscillating electric field $\mathbf{F}$, yielding the electron velocity $\mathbf{v}$ via integration:

$$
m \ddot{\mathbf{x}}=e \mathbf{F} \cos \omega t \quad \Rightarrow \quad \mathbf{v}=\dot{\mathbf{x}}=\frac{e \mathbf{F}}{m \omega} \sin \omega t+\mathbf{v}_{\mathbf{0}}
$$

The ponderomotive energy $U_{P}$ is defined as the mean oscillatory energy, and represents an important energy scale for the later discussion,

$$
U_{P}:=\frac{m}{2}\left\langle\dot{\mathbf{x}}^{2}\right\rangle=\frac{e^{2} \mathbf{F}^{2}}{4 m \omega^{2}}
$$

For a UV-wavelength of $\lambda=200 \mathrm{~nm}$ and an intensity of $1 \mathrm{~W} / \mathrm{cm}^{2}$, the ponderomotive energy is $U_{P}=4 \times 10^{-15} \mathrm{eV}$ - magnitudes below the measured values of few $\mathrm{eV}$.

Introducing the quantum picture of light-matter interactions [29], Einstein successfully 


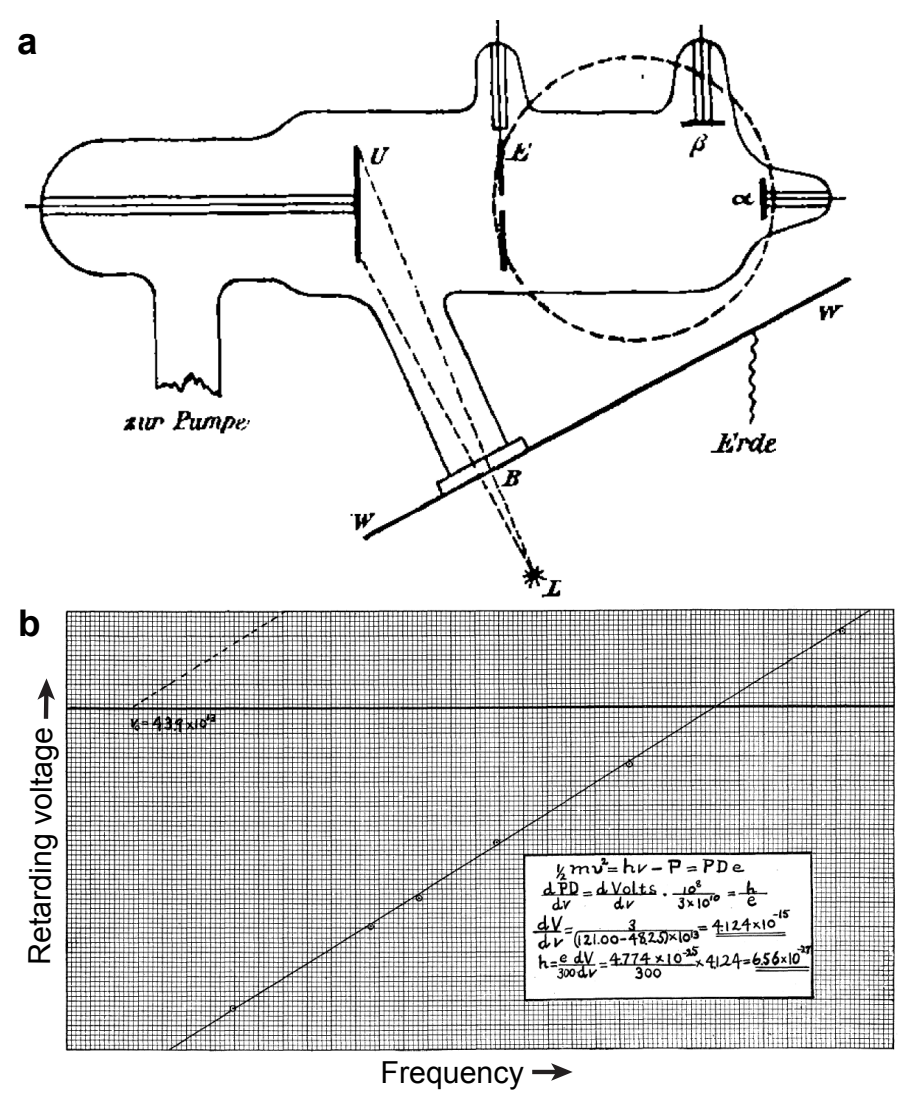

Figure 2.2: a) Sketch of Lenard's 1899 setup, displaying all components of a photoemission experiment: The light source $(L)$ is coupled via a suitable window $(B)$ inside a vacuum tube. Light hits the target $(U)$, inducing photoelectrons which are analyzed via magnetic fields (electromagnetic coil as dotted circle) or a retarding potential between $U$ and $E$. Induced electric charges are detected at $U$ and $\alpha$. Figure from Ref. [26]. b) The data of Millikan demonstrate Einstein's photoelectric law with a linear relation between frequency and electron excess energy (given as retarding potential in Volts). The slope determines Planck's constant $h$. Figure from Ref. [28].

explained the experimental findings, and was awarded with the Nobel-prize for his early application of quantum theory. Einstein's photoelectric law for the maximum excess energy upon photon absorption,

$$
E_{k i n}=\hbar \omega-\Phi,
$$

postulates a linear frequency-dependence of the electron excess energy, which was later experimentally confirmed by Millikan, see Figure 2.2b. It expains, moreover,

1. the presence of a frequency threshold for the emission $\hbar \omega_{\min }=\Phi$, given by the work function of the metal $\Phi$, 
2. no intensity-dependence of the electron energy.

Over the last hundred years, this linear photoemission process is widely applied as a versatile spectroscopic tool to study electronic properties of materials. The formation of photoemission spectra is typically understood as a three-step process, including (a) the photoexcitation of electrons inside the material, (b) the (short-range) transport to the surface and the accompanied production of secondary electrons via scattering, and (c) the escape through the surface barrier into the vacuum [30, pp. 14,15]. The nonlinear photoemission, investigated in this work, is primarily generated at the material surface and secondary electron contributions are negligible due to low excess energies prior the escape from the surface.

Whereas classical dynamics were discarded as the appropriate picture of photoemission under typical conditions, in principal, the classical concept of the electrodynamic interaction is certainly valid. As will be demonstrated in the following, the classical picture experiences increasing significance for the description of light-electron interactions however, under experimental conditions not available at the discovery of photoemission.

\subsection{Multiphoton absorption and the transition to strong-field light-matter interactions}

Already in his 1905 paper [29], Einstein mentions the possibility of quantum transitions via "multiple generating energy quanta" ${ }^{1}$ at high photon densities. The simultaneous absorption of multiple photons allows for quantum transitions inaccessible by single photon energies, see Figure 2.3a. The theoretical treatment of two-photon processes goes back to Goeppert-Mayer, who in 1930 quantum-mechanically derived the two-photon absorption probability in a time-dependent perturbation theory - and already emphasized the necessary high light intensities due to the quadratic intensity-dependence [31]. Consequently, it took laser radiation for the experimental demonstration [32] which was accomplished only twelve months after the first report on laser operation by Maiman in 1960 [33].

Generally, the multiphoton yield of $N$ th-order scales with the intensity $I^{N}$. For the ionization of gases, the nonlinear order $N$ is given by excitation frequency $v$ and the ionization potential $I_{P}, N \cdot h v \geq I_{P}$.

\footnotetext{
${ }^{1}$ In the original, p. 145 [29]: "[...] ein Energiequant des erzeugten Lichtes [kann] seine Energie von mehreren erzeugenden Energiequanten erhalten [...]".
} 

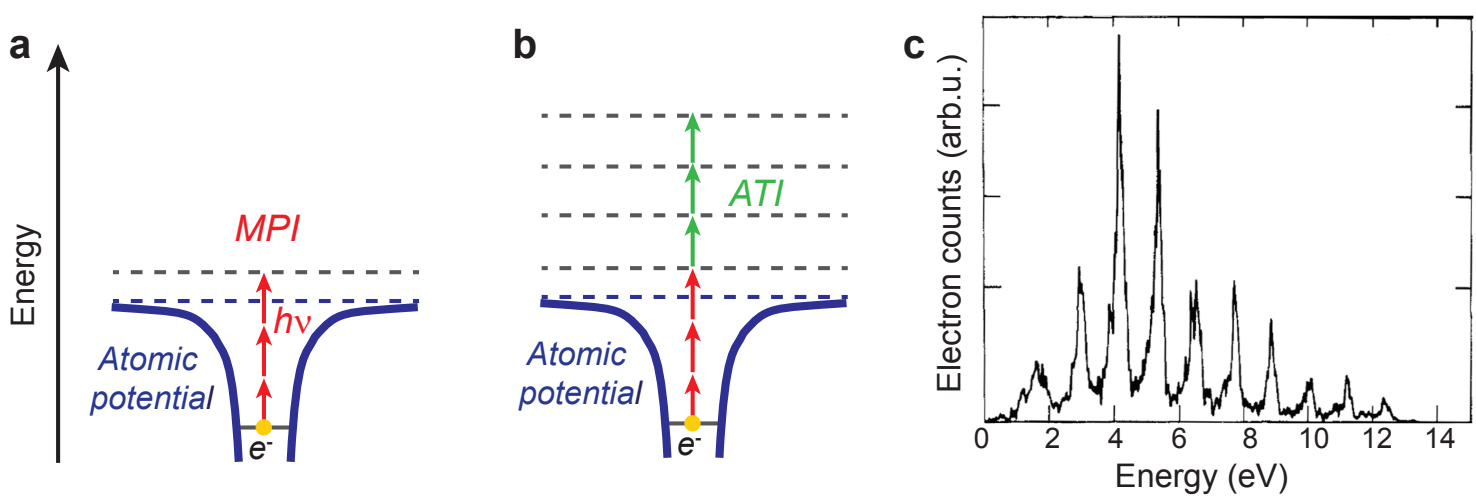

Figure 2.3: a) Multiphoton ionization (MPI) of an atom with photon energies below the ionization potential. b) For higher intensities, above-threshold ionization (ATI) is observed. c) ATI spectrum of xenon with $1064 \mathrm{~nm}$ light at $11 \mathrm{TW} / \mathrm{cm}^{2}$ and $130 \mathrm{ps}$ pulse duration, Figure from Ref. [34].

At more intense laser fields, experimental ionization studies revealed the absorption of more photons than required by the ionization potential [35], illustrated in Figures 2.3b,c. The additional absorption — viewed as "free-free" transitions within the unbound continuum - would violate the momentum and energy conservation for a single-electron-light interaction. This "above threshold ionization" (ATI) is now understood as the onset of the strong-field regime, where the light-field cannot be treated as a small perturbation of the potential, but significantly changes the final vacuum state. Keldysh characterized the transition between multiphoton ionization and strong-field ionization by introducing the ratio between the ionization potential and the ponderomotive energy [36]

$$
\gamma=\frac{\omega}{\omega_{t}}=\sqrt{\frac{I_{P}}{2 U_{P}}}=\frac{\omega \sqrt{2 m I_{P}}}{e F}
$$

Here, $\omega_{t}$ is the tunnel frequency, corresponding to the transition rate through the potential barrier of height $I_{P}$. At the transition to strong-field dynamics, the ponderomotive energy is comparable to the binding potential, $\gamma \approx 1$, representing the regime of ATI. Within the strong-field approximation, the final ionization states are described as so-called Volkovstates [37]. They represent plane-wave solutions of the Schrödinger equation, which are modulated by the classical vector potential in correspondence to classical electron oscillations, and are independent from the parent ion [38]. For a quantum transition to occur, the supplemental kinetic energy has to be supplied by the absorption of additional photons.

For long pulses ( $>500 \mathrm{fs}$ ), the increase in ionization potential is exactly regained as ki- 
netic energy due to the acceleration in the intensity-gradient of the laser focus. Such spectra exhibit distinct ATI peaks, which are separated by the photon energy (see Fig. 2.3c) [38]. In the short pulse regime, ATI signatures are more complex and reveal, e.g., a characteristic atomic sub-structure. The electrons regain only parts of the ponderomotive potential during their transition through the rapidly vanishing intensity gradient, resulting in intensity-dependent multiphoton resonances [38].

\subsection{Strong-field interactions I: Tunnel emission}

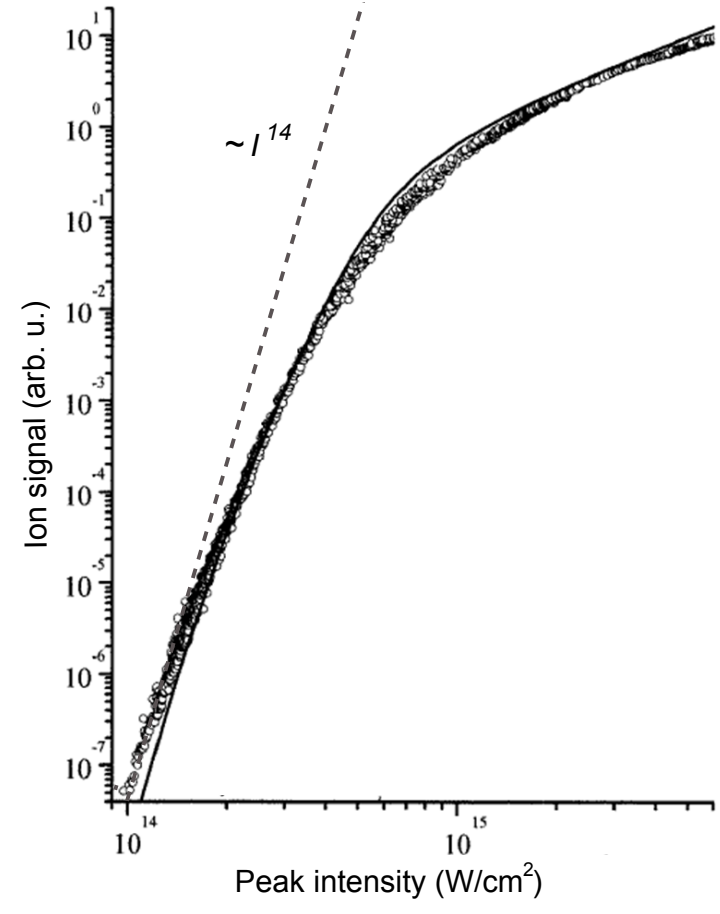

Figure 2.4: Multiphoton ionization of Ne using 200 -fs pulses at $800 \mathrm{~nm}$ wavelength. Experimental data (circles), ADK theory (solid line) and 14-photon slope (dashed line) in comparison. Figure adapted from Ref. [39].

Following Keldysh's rationale, the strongfield regime is distinguished by a breakdown of the perturbative picture and a sufficiently strong and steady distortion of the potential barrier. As derived in Ref. [36], under such conditions of high ponderomotive energy at $\gamma<1$, the multiphoton emission probability merges into a quantum-mechanical tunneling dependence.

Proposed in the early days of quantum mechanics $^{2}$, the classically-forbidden leakage or tunneling of an electron through the barrier was first studied for atomic ionization under the action of an intense static field [41]. Not long after the availability of intense coherent laser radiation, the characteristic exponential field-dependence of the atomic ionization yield was reported as an indication of strong-field ionization beyond the perturbative regime [42]. Figure 2.4 displays the intensity-dependent ionization yield of neon gas, and illustrates the increasing deviation from a multiphoton ionization

\footnotetext{
${ }^{2}$ The first reference of non-classical tunneling is actually found in a work of Friedrich Hund from 1927 on inversion-transitions of the $\mathrm{NH}_{3}$ molecule, deriving the probability of finding an electron on either side of the potential barrier [40].
} 
scaling for high intensities. Such tunnel ionization curves from atoms can be described by the expression from Ammosov, Delone and Krainov (ADK) [43].

At solids, field emission denotes the purely quantum-mechanical "extraction of electrons from cold metals by intense electric fields" [44], as defined in the prominent work of Fowler and Nordheim from 1928. The derivation of the static emission current density is based on the integration of the incident electron population over all energies and the weighting by a field- and energy-dependent barrier transmission function $T(E)$ which is calculated, e.g., via the Wentzel-Kramers-Brillouin (WKB) method [45]

$$
T(E)=\frac{4 \sqrt{E(\Phi-E)}}{\Phi+\mu} \exp \left(-\frac{4}{3} \sqrt{\frac{2 m_{e}}{\hbar^{2}}} \frac{(\Phi-E)^{3 / 2}}{e F}\right) \text {. }
$$

Here, $E$ is the electron energy above Fermi
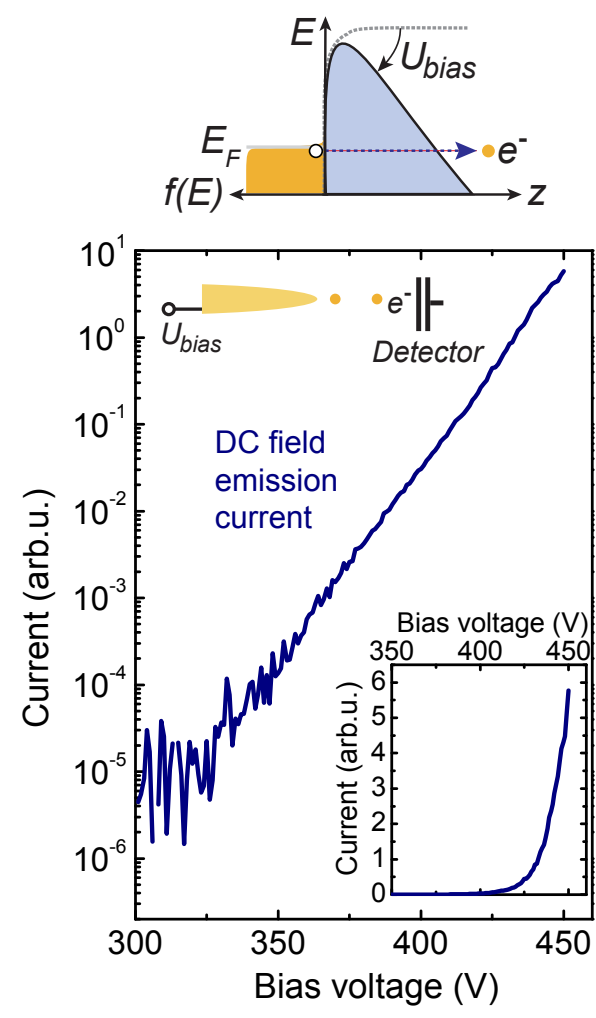

Figure 2.5: Static field emission for increasing bias voltage from a sharp gold nanotip, typically used in the experiments. level, $\Phi$ the work function and $F$ the electric field. The rigorous treatment of cold field emission results in the Fowler-Nordheim form of the field emission law for the current density $j$ [44]:

$$
j \propto F^{2} \exp \left(-\frac{4 \sqrt{2 m_{e}} \Phi^{3 / 2}}{3 \hbar e F}\right) .
$$

This derivation is based on a triangular barrier which is - in a more realistic model — rounded off by an additional image-potential term, resulting in a decreasing barrier height for increasing fields. The effect is accounted for via a fielddependent correction factor in the exponential term [45]. The high field strengths required are easily achieved at sharp metallic tips due to the geometric concentration of field lines at the apex. At bias potentials $U$, the field strength at the apex is given by

$$
F=\frac{U}{r \cdot k_{g e o}},
$$

$k_{\text {geo }}$ denotes a geometry dependent factor, typically 5-8 [46]. A characteristic tunneling 
curve for static field emission from an experimentally employed gold nanotip is displayed in Figure 2.5.

The treatment of field emission from solids at elevated temperatures requires the evaluation of the energy-dependent barrier transmission for a temperature-dependent FermiDirac distribution $f(E)$ [47]:

$$
f(E)=\frac{1}{\exp \left(\frac{E-E_{F}}{k_{B} T}\right)+1}
$$

For field emission in oscillating electric fields, the static tunneling process is considered to adiabatically follow the varying electric field up to optical frequencies. A delay of tunnel emission due to a finite response time of the tunneling wavefunction to establish is controversially discussed, see e.g. [48, 49], and novel experimental techniques are about to resolve the onset of tunneling from gases on timescales well below $1 \mathrm{fs}$, e.g., in Ref. [50].

AC-field emission from metal tips in intense low-frequency fields has actually been confirmed experimentally before the broad availability of laser radiation in powerful microwave resonators [51]. Due to the exponential tunnel dependence on the field strength, tunneling occurs in a narrow temporal window around the oscillation maxima, resulting in the emission of short electron bursts within the microwave sub-cycle [52]. The investigation of optical field emission at higher infrared frequencies are subject of this work, and bears the potential for sub-fs electron pulses [53]. As apparent from the discussed sensitivity of field emission to the momentary electron population, ultrashort field emission can also be applied in pump-probe experiments to temporally resolve carrier dynamics in solids - in analogy to time-resolved photoemission [54], and will be demonstrated in Chapter 6.

\subsection{Strong-field interactions II: Post-ionization dynamics}

In addition to the liberation of a bound electron from an atomic potential via processes of ionization or photoemission, a wide variety of strong-field phenomena requires the consideration of subsequent interactions between the electron, the light-field and the parent atoms after emission. A "Simple man's model" was introduced to describe the dynamics of the emitted electronic wave packet by classical mechanics, and is based on the trajecto- 
1. Tunnel ionization

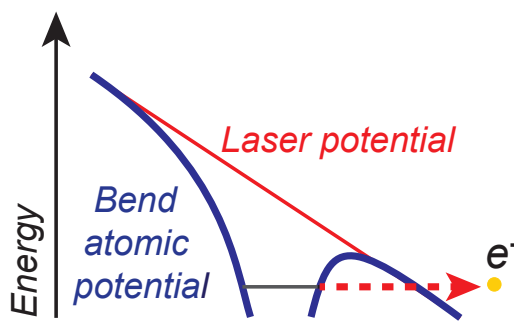

2. Acceleration

3. Recombination

Figure 2.6: Semi-classical three-step model of high-harmonic-generation. 1.) After tunnel ionization in the distorted atomic potential, the electron wavepacket is accelerated in the laser electric field and driven back to the parent ion in the next oscillation half-cycle (2.). 3. The electron recollides with the parent ion, resulting in a rapidly oscillating dipole and the emission of coherent high harmonic radiation.

ries of point charges in the oscillating laser electric field [5]. An important application is the description of high-harmonic generation (HHG) via a semi-classical three-step model, see Figure 2.6. After multiphoton or tunneling ionization of a gas atom, the electron is accelerated, driven back in the next oscillation half-cycle of the laser field and re-collides with the parent ion [6]. The interference between the quasi-free wave packet and the parent wavefunction of the ion results in a rapidly oscillating dipole, radiating at typically up to hundred harmonics of fundamental frequency. The highest photon energy $E_{\text {HHGmax }}$ is given by the maximum recollision energy of the classical trajectory, yielding the linear dependence on the ponderomotive energy $E_{H H G m a x}=3.17 U_{P}+I_{P}$. As apparent from the wavelength scaling of the interaction, driving the HHG process with long wavelengths enables highly energetic radiation, as recently demonstrated with a fundamental wavelength of $3.9 \mu \mathrm{m}$ and resulting cutoff energies of $h v=1.6 \mathrm{keV}$ in the X-ray spectrum ${ }^{3}$ [55].

For strong-field atomic ionization, photoelectron energy distributions can be derived according to a semi-classical two-step model. In the first step, the electrons are liberated from the atomic potential via adiabatic tunneling, and in the second step the electrons are emitted, either directly or after rescattering with the parent ion. The electron kinetic energy spectra are obtained by weighting the phase-dependent final kinetic energies with the nonlinear tunnel emission rate, see Figure 2.7a,b. The classical analysis yields electron energies up to $2 U_{P}$ for direct emission, and up to $10 U_{P}$ for rescattered electrons [7]. As apparent from the calculation in Figure $2.7 \mathrm{~b}$, the maximum yield at zero phase directly results in electron spectra peaked at zero kinetic energy, and in an energetic spread

\footnotetext{
${ }^{3}$ Regarding generation efficiency, shorter driving wavelengths are generally favored.
} 

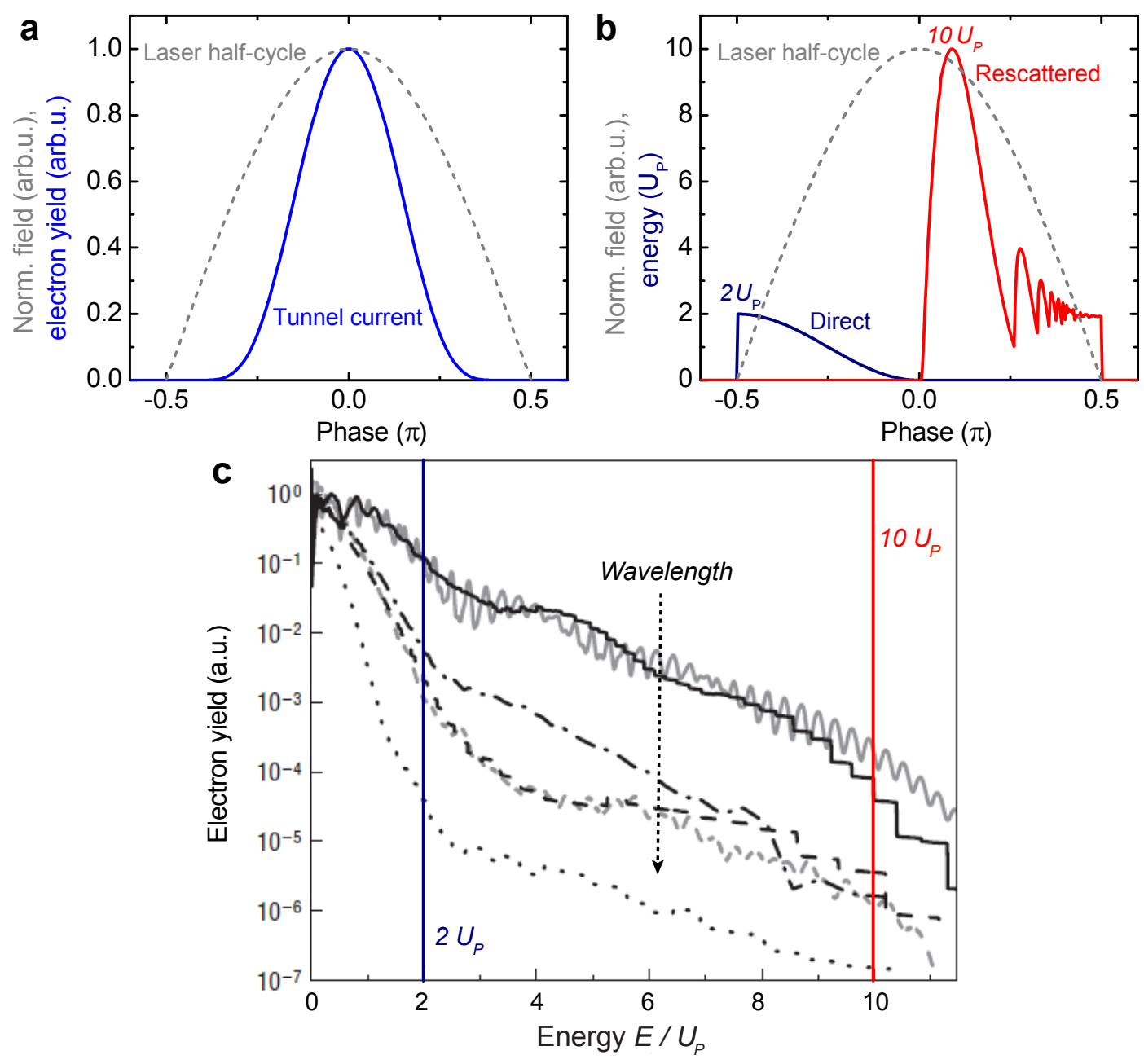

Figure 2.7: a) In the first step of a semi-classical model of strong-field photoionization, the tunnel yield is derived for all phases of the laser field. In the limit of adiabatic tunneling and due to the emission nonlinearity, the electron yield is temporally confined to phases around the peak field. b) In the second step, electron trajectories are calculated by classical particle propagation in the laser electric field. The final electron kinetic energies are evaluated for all emission phases. Electrons emitted before the peak field are directly emitted, and the maximum energy is given by $2 U_{P}$. Electrons emitted after the peak field are driven back to the atom and can gain energies up to $10 U_{P}$ after elastic re-scattering. The oscillatory features at later phases are caused by the interaction with subsequent cycles. c) Wavelength-dependent strong-field ionization of argon at a fixed intensity: The experimental photoionization spectra at $0.8 \mu \mathrm{m}, 1.3 \mu \mathrm{m}, 2 \mu \mathrm{m}$ and $3.6 \mu \mathrm{m}$ (black lines, top to bottom) are normalized with respect to the ponderomotive energy. For higher wavelength, the universal $2 U_{P} / 10 U_{P}$ energy cutoffs from the classical analysis for direct and rescattered electrons, respectively, are increasingly pronounced. Grey lines show simulations. Figure c) from Ref. [56]. 
up to $10 U_{P}$ due to the sensitive dependence of the final kinetic energy of the rescattered electrons around zero phase. Experimental ionization studies with intense, tunable excitation from the near- to the mid-infrared demonstrate the validity of the strong-field approximation for atoms and the convergence to the cutoff-energies from "Simple-man's" predictions [56] at long wavelengths, as shown in Figure 2.7c.

\subsection{Strong-field dynamics at nanostructures}

As proposed by Keldysh, the observation of tunnel emission from solids is favored in comparison to gases due to the faster energy dissipation, higher breakdown fields and lower ionization potentials [36]. However, the experimental investigation of strong-field dynamics at solids remains challenging, as described below.

Optical nonlinearities in transparent media induce self-focusing, nonlinear frequencymixing and multiphoton excitations which often result in material damage upon pulse propagation. A broad spectral range from ultraviolet to optical frequencies is efficiently absorbed in metals, and the deposition of energy results in nonthermal excitations, electron heating, thermionic emission and ultimately in melting. The nonthermal electron populations thermalize within $10-100 \mathrm{fs}$ to hot electron distributions with lifetimes of several ps, see Appendix 8.2. At additional high static bias, such excited electrons can tunnel at an enhanced transition rate (laser-enhanced photoemission) or hot electrons directly pass above the potential barrier (thermionic emission). The transition between instantaneous and thermal emission processes may be gradual, and the careful distinction between these regimes is important for the generation of ultrashort electron pulses.

Direct photoemission with photon energies $h v>\Phi$ from dense macroscopic structures results in a large number of photoelectrons, and their spectral analysis is complicated by electron-electron interactions between the emitted electrons and by space-charge fields. At infrared frequencies $h v<\Phi$, the nonlinear photoemission is highly susceptible to surface protrusions which form emission hotspots and often mask the surface signal.

The experiments reported here employ nonlinear photoemission from geometrically well-defined single nanotips, see Section 3.8. The structures feature smooth shaft geometries extending over the excitation focal waist, confinement of the emission to the few-nm sharp apex and rapidly diverging electric field lines that reduce the sensitivity to spacecharge effects.

At such metallic nanotips, several nonlinear and strong-field emission processes have 
been observed previously. For moderate near-infrared ( $800 \mathrm{~nm}$ wavelength) excitation of gold nanotips, multiphoton photoemission represents the dominant ultrafast emission process. A photon energy of $h v=1.55 \mathrm{eV}$ and a work function of $\Phi=5 \mathrm{eV}$ implies the simultaneous absorption of at least 4 photons, which is experimentally confirmed in Ref. [57], and similarily for tungsten in Ref. [58]. Notably, this nonlinear emission process in combination with the field-enhancement at the apex of the nanotip provides for an emission site which is not determined by the illumination focus, but by the local field distribution at the tip surface. As a result, the source brightness $B=J / \Omega A_{\text {em }}$ and the coherence [59] of this highly localized emitter are significantly improved compared to planar photocathodes. Here, $J$ is the photocurrent, $\Omega$ is the solid angle of emission and $A_{e m}$ the prospective nanometric emission area. Such emitters are highly desirable as high-brightness and coherent electron sources for novel ultrafast electron imaging and diffraction instruments [12, 13, 14].

Photoemission studies with the additional application of high static bias fields demonstrate the static tunneling of ultrafast non-thermal electronic populations [57, 62].

At more intense NIR-illumination, characteristic ATI-signatures at $\gamma \approx 3$ have been reported from tungsten nanotips in Ref. [63]. The ATI-spectra indicate the absorption of up to 6 additional photons - even with low energy pulses due to the field enhancement ${ }^{4}$ at the tip apex.

A hallmark of strong-field interactions is the sensitivity to the absolute carrier-envelope phase (CEP) which determines the exact temporal evolution of the electric field [69]. Experiments with CEP-stabilized few-cycle NIR-pulses, reveal the impact of the CEP onto photoemission spectra from tungsten and gold nanotips at $\gamma \approx 1$ [70, 71]. Photoemission spectra are demonstrated to vary with the CEP-phase, attributed to the interference between two successively emitted, rescattered electron wave packets ("temporal doubleslit") [70] and to CEP-sensitive strong-field acceleration [71].

At the transition to the strong-field regime $(\gamma \approx 1)$, the constant slope of the intensitydependent multiphoton emission yield is found to decrease for NIR-illumation of gold nanotips, see Figure 2.8a [60]. At high intensities, transitions or channels of low-order photoemission are suppressed due to the scaling of the final state energy with the pon-

\footnotetext{
${ }^{4}$ Strong-field dynamics in the enhanced plasmonic near-field at nanoantennas and funnels with significant high-harmonic generation rates were reported for moderate pulse energies from laser oscillators $[64,65]$. However, recent studies identified strong-field excitation but subsequent incoherent atomic fluorescence as the predominate EUV-generation process in nanoscopically confined generation volumes $[66,67,68]$.
} 

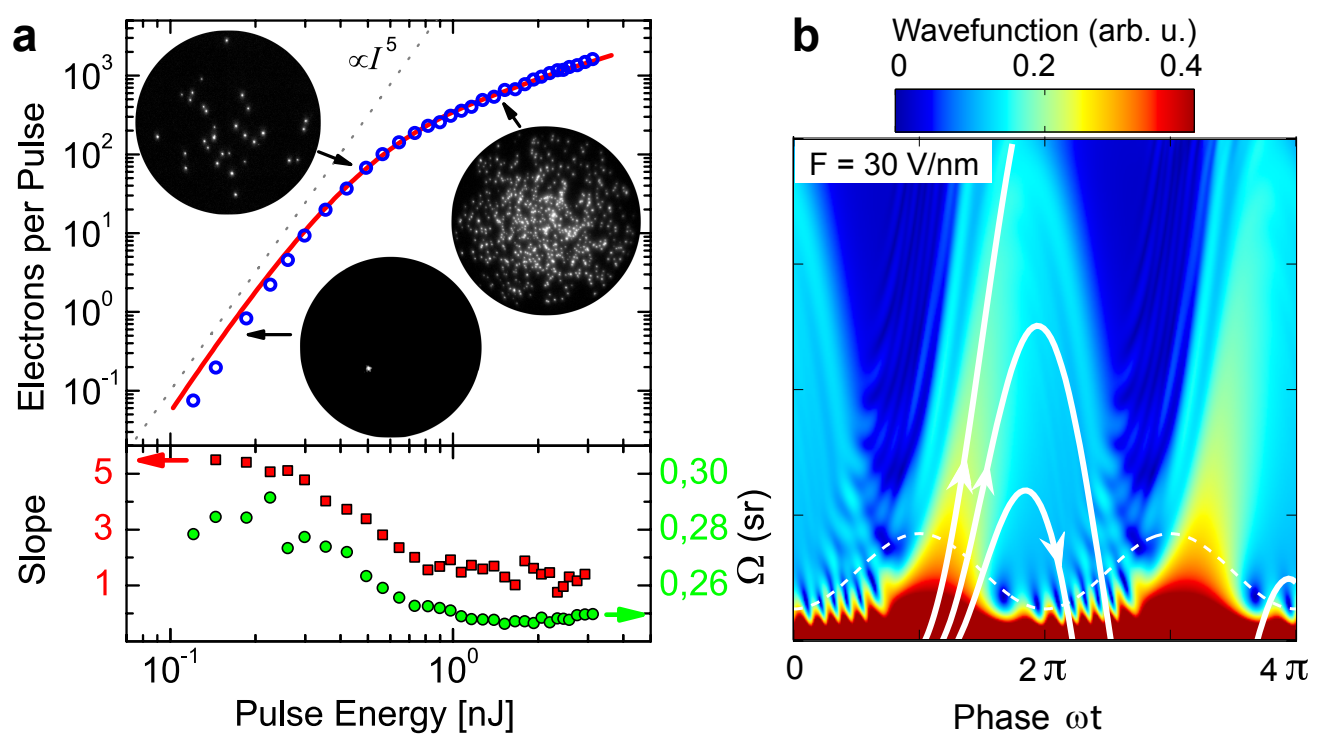

Figure 2.8: a) Transition to strong-field emission from gold nanotips at $800 \mathrm{~nm}$ wavelength. At high pulse energies, the constant multiphoton emission slope decreases around $\gamma \approx 1$ due to a superposition of the closing of lower-order and the opening of higher-order emission channels. This is accompanied by a reduction in the emission angle $\Omega$ due to forward acceleration. Figure from Ref. [60]. b) Numerical solution of the Schrödinger equation for NIR-induced photoemission from a gold surface. At the high field strength, the temporal structure of the driving field (dashed) is imprinted onto the wavefunction. The emission is governed by the tunneling at the peak field and classical propagation of the wavepacket (solid white trajectories). The small phase delay is due to a non-zero excess energy. Courtesy of S. V. Yalunin, adapted from Ref. [61].

deromotive potential. In the combination of strong-field closing of lower-order channels and opening of higher-order channels, the total yield decreases. Additionally, the emission cone narrows due to the increase in forward momentum due to the laser acceleration. Further access to Keldyh parameters substantially below unity and optical field emission (OFE) may not be accessible in the near-infrared due to material damage [60, 72], but at longer wavelengths.

The numerical solution of the Schrödinger equation for NIR-photoemission at a solid gold surface in Figure $2.8 \mathrm{~b}$ illustrates photoemission dynamics that actually adiabatically follow the laser electric field. The detailed theoretical description is given in Ref. [61]. At sufficient field strength, the wavefunction and the corresponding current density are in phase with the driving field, suggesting that the photoemission process can be described in analogy to the two-step model of photoionization via optical field emission and classical 

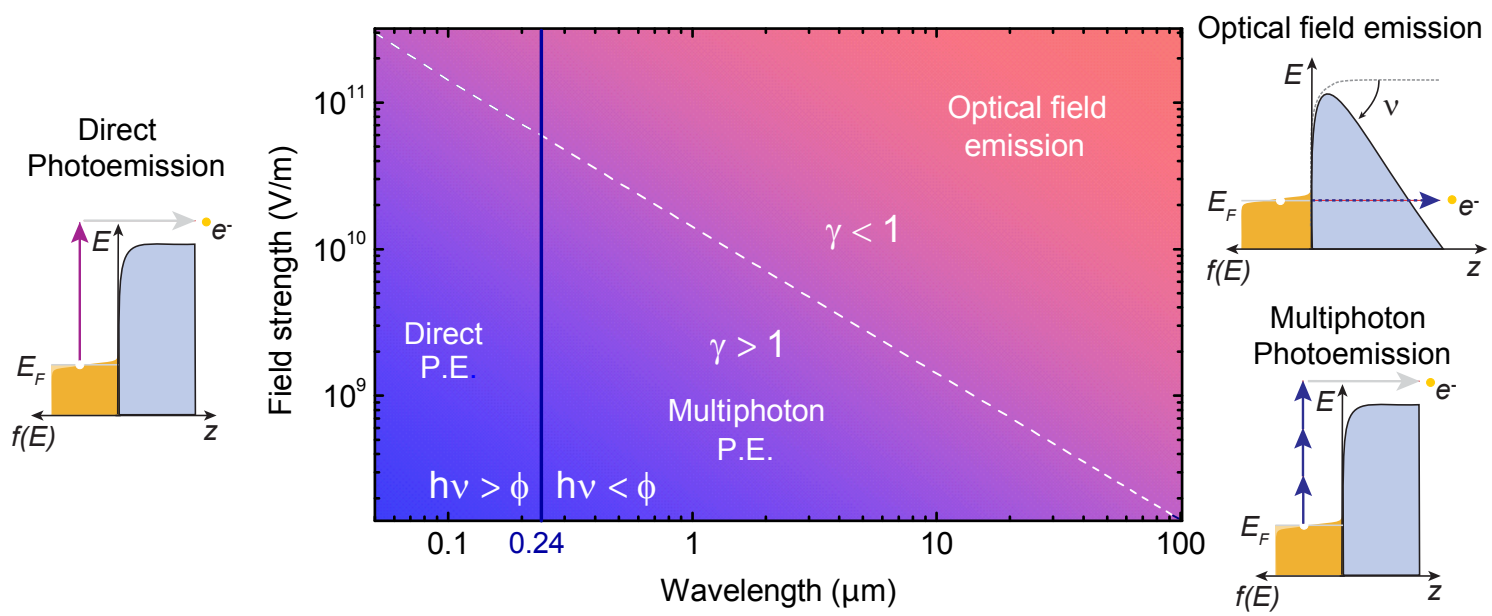

Figure 2.9: Ultrafast photoemission regimes as a function of excitation frequencies and field strengths, assuming an ionization potential or work function of $5 \mathrm{eV}$. Direct photoemission is encountered at $h v>\Phi$ (Schematic left). For sufficient field strength, multiple photons simultaneously induce nonlinear photoemission (Schematic right). The transition to optical field emission is marked by a Keldysh-parameter of unity (diagonal line), achieved at strong and long-wavelength excitation (Schematic right).

propagation in the laser field. Remarkably, such classical electron dynamics - soughtafter in the early days of photoemission - seem to finally emerge as the appropriate description at the strong-field excitation conditions available today.

Figure 2.9 presents the regimes of instantaneous photoemission, encountered for particular field strengths and wavelengths - namely, the direct photoeffect, multiphoton emission and optical field emission. The distinction is based on a typical metallic work function of $\Phi=5 \mathrm{eV}$ and the respective Keldysh parameter. 


\section{Chapter 3}

\section{Experimental methods and concepts}

This chapter introduces the experimental background of the presented studies. The experimental realization is based on commercial ultrafast laser sources and on custom-built ultrafast spectroscopy and vacuum setups, implemented over the course of the thesis. Here, we briefly refer to the device development, sketch design features and provide technical specifications on key components. Moreover, the nanostructure preparation and characterization are described, and the concept of optical streaking is introduced.

\subsection{Generation of femtosecond pulses - ultrafast laser sources}

Ultrafast spectroscopy with sub-femtosecond temporal resolution and strong-field physics at peak intensities of multiple $\mathrm{TW} / \mathrm{cm}^{2}$ are directly connected to the advances of ultrafast laser technology. Modern sources, based on Kerr-lens mode-locked oscillators with titanium-doped sapphire (Ti:Sa) as the laser-active medium [73, 74], are capable of delivering few-cycle pulses below $5 \mathrm{fs}$ duration at a central wavelength of $800 \mathrm{~nm}$ [75]. Nanojoule pulse energies from oscillators are amplified up to the joule-level via chirpedpulse-amplification $[3,76]$.

The experiments reported in Chapter 4 are based on a Ti:Sa oscillator-amplifier system, providing 30-fs pulses with $3 \mathrm{~mJ}$ pulse energy (femtosecond-oscillator Ti-Light, multipass amplifier Odin-II, Quantronix, USA). The stretched oscillator pulses are amplified in an eight-pass pre-amplifier stage [77], temporally gated from a repetition rate of $80 \mathrm{MHz}$ to $1 \mathrm{kHz}$ and further amplified in a double-pass power-amplifier stage.

Prior to the measurements of Chapters 5,6, the unit was replaced by a system delivering $4.2 \mathrm{~mJ}$ pulse energy at $50-\mathrm{fs}$ pulse duration and $1 \mathrm{kHz}$ repetition rate (femtosecond- 


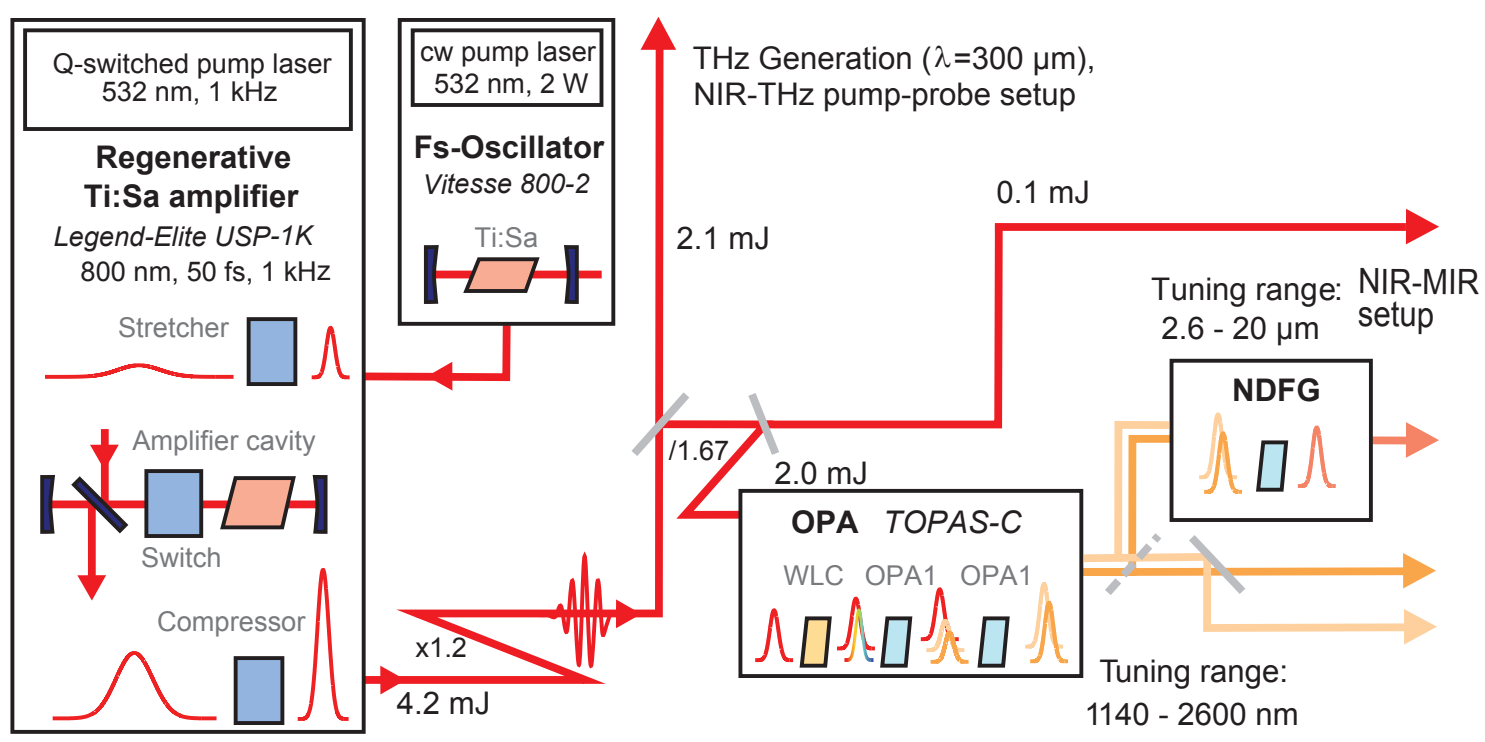

Figure 3.1: Layout of the ultrafast laser source. The subsequent steps for the generation of tunable ultrafast pulses are illustrated via the basic optical components. The oscillator-amplifier system delivers 50 -fs pulses at $1 \mathrm{kHz}$ repetition rate and $800 \mathrm{~nm}$ wavelength. The beam sizes are adjusted via optical telescopes, and the light is divided into different arms using dichroic beamsplitters. Half of the energy is directed to the $\mathrm{THz}$ setup, the second part is mostly used to pump the optical parametric amplifier (OPA). Signal and idler waves are employed either for difference frequency generation (NDFG), or are split to be individually accessed.

oscillator Vitesse, regenerative amplifier Legend Elite, Coherent Inc., USA), see Figure 3.1. The higher pulse energy is achieved using a regenerative amplifier cavity with a single Ti:Sa crystal, cooled to $-10^{\circ} \mathrm{C}$.

The pulses are characterized by a fiber-coupled CCD spectrometer and with the method of spectral phase interferometry for direct electric field reconstruction [78] (LX Spider, A.P.E GmbH, Germany).

\subsection{Frequency-tunable pulses via nonlinear optics}

Photoemission studies over a broad frequency range require the derivation of tunable excitation spectra from the fundamental NIR pulses under preservation of pulse duration and coherence properties. Coherent frequency conversion is achieved via nonlinear optics, employing anharmonic oscillations of strongly-driven bound electrons in suitable nonlinear media. The resulting nonlinear relation between the incident optical electric field and the induced macroscopic polarization $P_{M}$ can be expanded into a power series of 

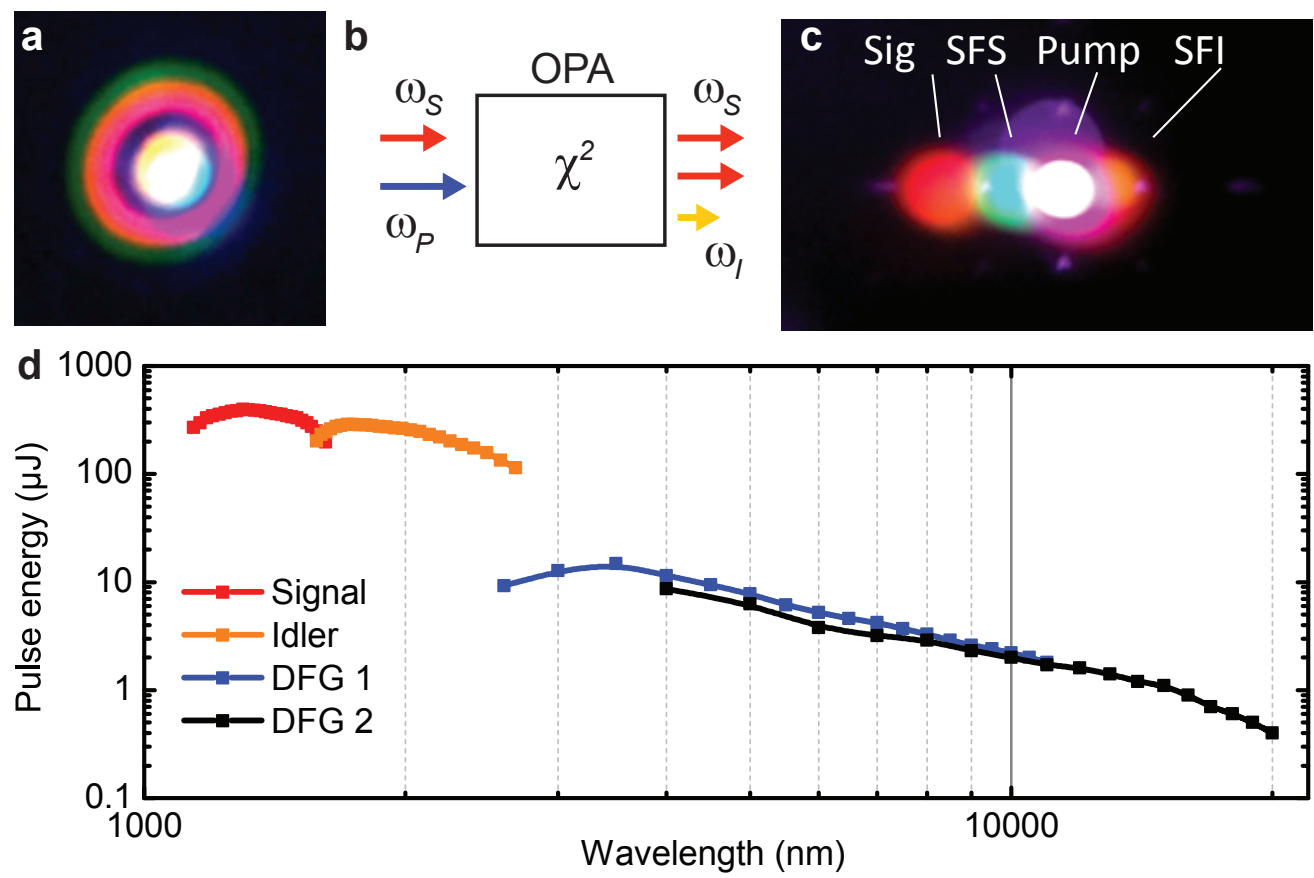

Figure 3.2: a) Color photograph of the white-light beam (diffuse reflection), which is generated in a sapphire plate and coherently seeds the OPA. b) The nonlinear interaction of a weak signal at frequency $\omega_{S}$ and a strong pump at $\omega_{P}$ induces the splitting of the pump photon into an additional signal photon at $\omega_{S}$ and an idler wave at $\omega_{I}$. The lengths of the arrows indicate the photon energies. c) Color photograph (diffuse reflection) of the output from the first OPA stage displays the signal and the white-light waves (Sig), and the sum frequency of pump and signal (SFS) in the visible arising from further nonlinear mixing, the residual intense pump (Pump), and the sum frequency of pump and idler (SFI). d) Output energies in the tuning range, accessed with OPA signal and idler, and DFG $1\left(\mathrm{AgGaS}_{2}\right)$ and DFG $2(\mathrm{GaSe})$ for a pump energy of $2 \mathrm{~mJ}$.

the harmonic driving field $F$ [79]:

$$
P_{M}=\varepsilon_{0} \cdot\left(\chi_{1} F+\chi_{2} F^{2}+\chi_{3} F^{3}+\ldots\right)
$$

The lowest order nonlinearity $\chi_{2}$ couples three optical waves and gives rise to the nonlinear frequency conversions of second harmonic generation (SHG), sum frequency generation (SFG), optical rectification (OR), and difference frequency generation (DFG) or noiseless optical parametric amplification (OPA). Compared to harmonic generation schemes, OPA enables frequency tuning over a continuous spectral range [80].

The optical parametric amplifier employed in this work (Topas-C, Light Conversion Ltd., Lithuania) covers a wavelength range of 1140 - $2600 \mathrm{~nm}$ with a conversion efficiency 
up to $40 \%$. Depending on the configuration, the system is pumped via $1.5,2$ or $2.8 \mathrm{~mJ}$ pulse energy from the Ti:Sa system, sketched in Figure 3.1. A small fraction of the pump wave generates a white-light continuum (WLC) in a sapphire plate (see Figure 3.2a) that coherently seeds the parametric amplification in two successive BBO crystals. In analogy to the DFG process, a weak input signal at frequency $\omega_{S}$ interacts with a strong pump wave at frequency $\omega_{P}$, and induces the splitting of the pump photon energy. This results in the amplification of the signal wave and the generation of an idler wave, see Figure 3.2b,c. Continuous tuning is archived by selective amplification of the signal band via phasematching with the crystal angle and via the temporal delay between seed and pump pulses.

Further access into the mid-infrared spectral region up to $20 \mu \mathrm{m}$ wavelength is provided by difference frequency generation between the signal and idler waves [81]. The noncollinear DFG stage covers the wavelength ranges of 2.6 - $5 \mu \mathrm{m}$ (in $\mathrm{AgGaS}_{2}$ ) and 4 $20 \mu \mathrm{m}$ (in GaSe), see Figure 3.2d.

\subsection{Infrared beam characterization}

In contrast to the widespread application of photodetectors for the energetic, spatial and temporal beam characterization at UV, visible and near-infrared frequencies, the characterization of low-energetic infrared beams is much more challenging. Suitable highsensitivity devices are typically based on thermal detection and, thus, are sensitive to the thermal background radiation, often require active-cooling and are less available compared to detectors for higher photon energies. In the following, we present the experimentally implemented characterization schemes.

Average power measurements down to the $100 \mu \mathrm{W}$ level are provided by thermopile detectors with a flat spectral response from the visible to the mid-IR (S302C, Thorlabs, USA). Employing the temperature-dependent polarity of $\mathrm{LiTaO}_{3}$ thin-films, appropriate pyro-electric detectors are sensitive to fast changes in the absorbed power, and submillisecond response times enable single-shot, pulse-to-pulse energy measurements at few nJ-level (Energy detector QE8SP-B-MT, Gentech-EO, Canada).

Beam tracking and alignment at long mid-IR wavelengths are facilitated by a thermal infrared-camera (EasIR-4, Guide-infrared, Wuhan, China) employing germanium optics and a 160 x 120 pixel micro-bolometer-array with specified sensitivity in the range of 8-14 $\mu \mathrm{m}$.

Characterization and optimization of infrared focusing is achieved by scanning a razor 
a

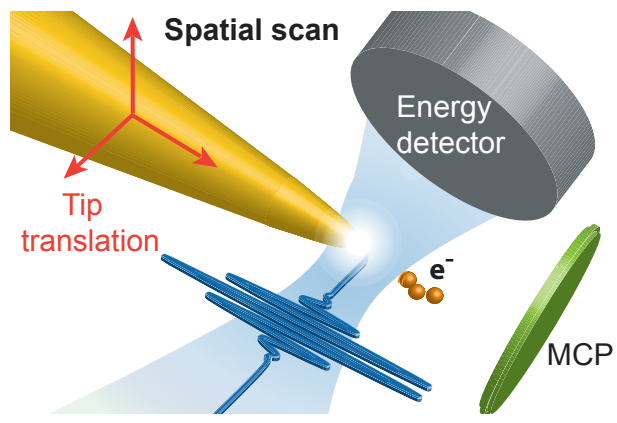

b

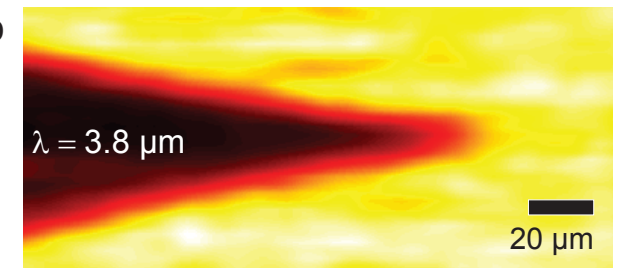

C

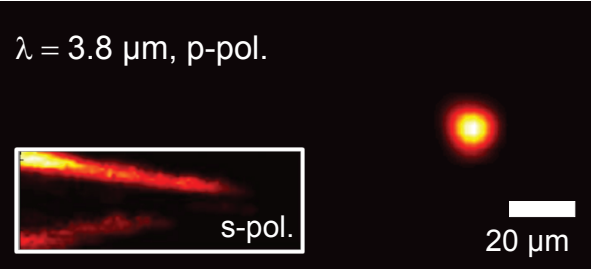

d

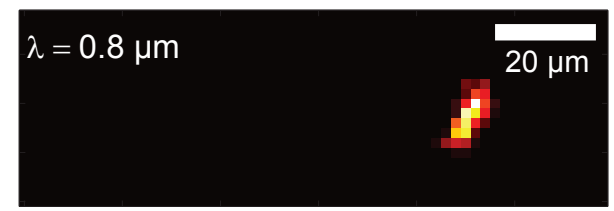

e

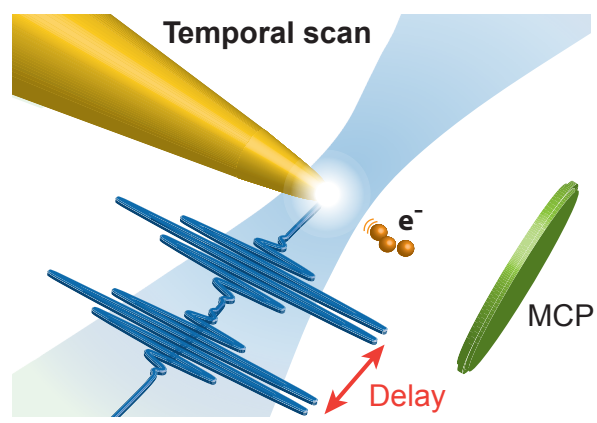

f

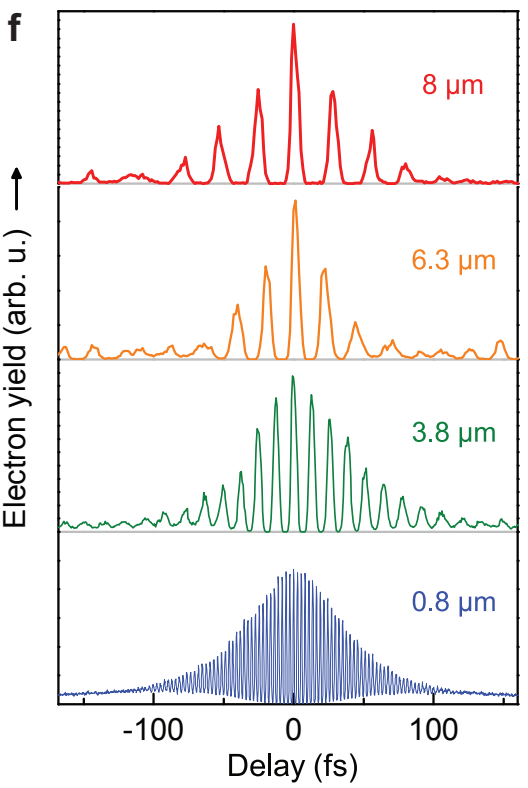

Figure 3.3: a) Spatial beam characterization in the infrared via scanning the nanotip in the focal plane. The transmitted light (b) at each tip position forms a shadow image of the tip, shown at $3.8 \mu \mathrm{m}$ wavelength. The simultaneously recorded photoemission yield (c) maps the focal size; d) a corresponding measurement at $0.8 \mu \mathrm{m}$. Only for a polarization parallel to the tip, the emission signal is localized at the apex, as illustrated by mapping the emission from the shaft at perpendicular polarization (c, inset). e) Temporal characterization of near- and mid-infrared pulses via autocorrelation with the nonlinear electron yield. A pulse and a delayed copy are coupled onto the nanotip, f) the electron yield is recorded as a function of the delay for various wavelengths.

blade through the focus and recording the transmitted energy (knife-edge-method) with a pyro-electric detector. Similarly, a two-dimensional scan of the nanotip through the focus generates shadow images, shown in Figures 3.3a,b. The intensity distribution represents a convolution of the laser focus with the structure profile. Such shadow images provide the nanostructure position with respect to the laser focus, and their minimum feature size is a 
measure of the laser focal size. The latter is directly mapped by simultaneously recording the photoemission yield from the nanotip apex, displayed in Figures 3.3c,d. The emission spot represents the convolution of the laser focus with the spatially localized nonlinear apex emission, and allows for focus determination and optimization. The focal diameters are found to range from $3-16 \mu \mathrm{m}$ between 0.8 and $8 \mu \mathrm{m}$ wavelengths.

The temporal characterization of ultrashort pulses relies on the temporal gating with a second equally short pulse or with an identical copy of the pulse itself. The interaction is facilitated via nonlinear cross- or auto-correlation, typically employing up-conversion via SFG, SHG and two-photon-absorption $[82,83]$. Compared to visible and near-infrared frequencies $[78,84,85]$, pulse characterization in the mid-IR is further complicated by the fact that the relative bandwidth $\Delta f / f$ of short pulses grows with the center frequency $f$. For instance, the relative bandwidth of transformation-limited 50 -fs pulses at $800 \mathrm{~nm}$ of $\Delta f / f=0.02$ increases to $\Delta f / f=0.6$ at $20 \mu$ m wavelength $^{1}$. Thus, optimized nonlinear detection crystals supporting high transmission, low dispersion and phase-matching over a broad spectral window around the central frequency have to be found. Typically, a set of different crystals is required to cover the tuning range continuously [82].

A different approach, implemented in this work and presented in Chapter 4, employs the instantaneous nanotip-photoemission as a broadband nonlinearity. Following an interferometric autocorrelation (IAC) scheme, infrared pulses and variably-delayed copies are coupled onto the apex of the nanotip, illustrated in Figure 3.3e. Recording the photoemission yield $I_{P C}(\tau)$ as a function of the relative pulse delay $\tau$, represents the autocorrelation:

$$
\operatorname{IAC}_{P E}(\tau) \propto \int_{-\infty}^{\infty} d t(F(t)+F(t-\tau))^{g(F(t)+F(t-\tau))} .
$$

The correlation nonlinearity $g$ depends on the emission process and may not be a constant value but instead varies with the field strength, as in the case of the tunneling nonlinearity. The calculated autocorrelation traces in Figure 3.4 for increasing nonlinear order $g$ illustrate the impact onto the IAC-traces. The peak-to-baseline ratio grows with increasing nonlinear order as $2^{g-1}$, given by the nonlinear signal of the sum of the fields, $I A C \propto(E+E)^{g}$, and the sum of the individual signals, IAC $\propto E^{g}+E^{g}$, at large delays. The experimental pulse durations are extracted from the intensity dependent IAC-traces via numerical fitting of the field-emission dependence, yielding durations between 60 and $95 \mathrm{fs}$, see Section 4. This method is applied over a wavelength range up to $14 \mu \mathrm{m}$, and

\footnotetext{
${ }^{1}$ Given the time-energy uncertainty for Gaussian pulses: $t_{p} \cdot \Delta f=0.44$.
} 

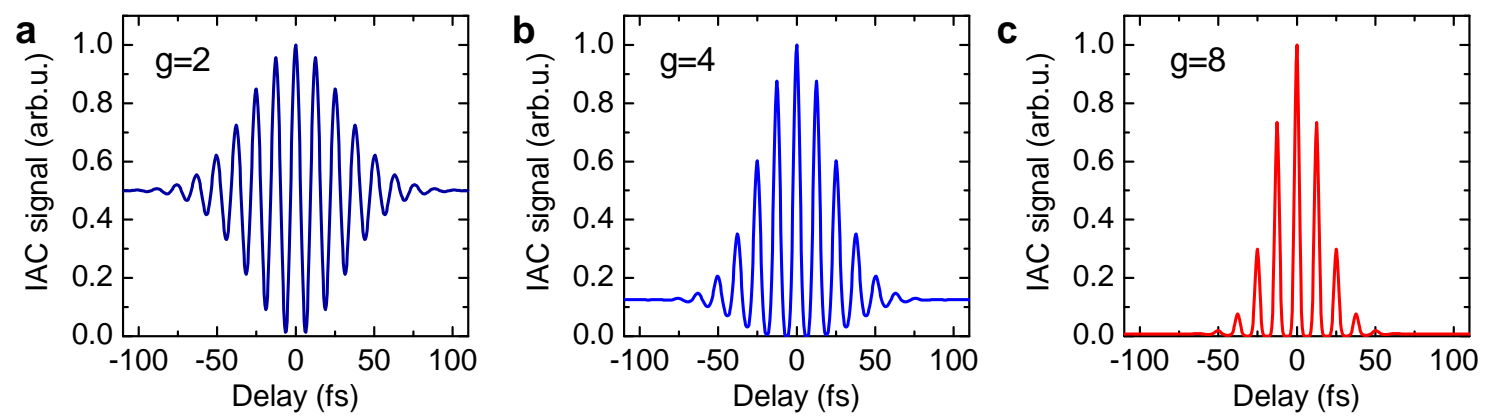

Figure 3.4: Simulated interferometric autocorrelation traces for growing nonlinear order. The normalized traces display the increase in the peak-to-baseline ratio and the sharpening of the oscillations. The pulse length is $50 \mathrm{fs}$ at $3.8 \mu \mathrm{m}$ wavelength. a) $\mathrm{g}=2$ represents the case of a linear field-correlation, detected as an intensity. This IAC only contains spectral information. b) The widely employed nonlinear field-correlation via second harmonic generation corresponds to $\mathrm{g}=4$ and is sensitive to a pulse-chirp. c) Four-photon photoemission yields $\mathrm{g}=8$.

can, in principle, continuously cover the full infrared spectrum, spanning from optical to radio-frequencies and even static fields.

\subsection{Generation of ultrashort Terahertz transients}

The development of devices for the far-infrared spectral region - between infrared laseroptics and microwave electronics $(0.1-10 \mathrm{THz}, 30-3000 \mu \mathrm{m})$ - is driven by rapidly evolving applications of $\mathrm{THz}$ sensing and imaging [86, 87, 88, 89].

Sources based on femtosecond-lasers provide coherent few-cycle $\mathrm{THz}$ pulses of picosecond duration and established the fields of $\mathrm{THz}$ time-domain and time-resolved spectroscopy $^{2}$. Significant advances during the last two decades enable high-field table-top $\mathrm{THz}$ sources with peak fields of several $\mathrm{MV} \mathrm{cm}^{-1}[89,90]$, opening up the regime of nonlinear THz optics [91, 92]. The key generation concepts rely on a) carrier acceleration in photoconductive switches [93], b) optical rectification in nonlinear crystals $[89,94]^{3}$ or c) laser-driven acceleration of plasma electrons $[95,96]$. The latter method is employed in this work and substantially extends the experimentally covered frequency range. Providing intense and exactly reproducible ultrashort waveforms, this source is exceptionally useful for the observation and the direct control of field-driven processes.

\footnotetext{
${ }^{2}$ Time-domain spectroscopy denotes the measurement of static optical properties via direct detection of transmitted or reflected amplitudes and phases. Ultrafast time-resolved spectroscopy relies on pump-probeschemes, accessing dynamic properties.

${ }^{3}$ Very recently, peak fields of up to $0.6 \mathrm{GV} / \mathrm{m}$ at $3 \mathrm{THz}$ are reported, using organic DSTMS crystals.
} 

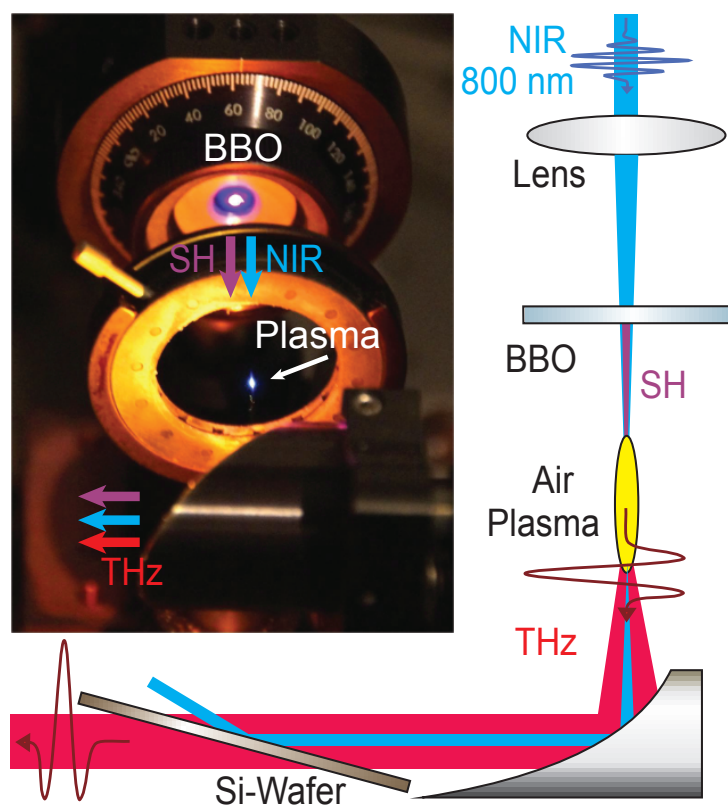

Figure 3.5: Schematic of $\mathrm{THz}$ generation in an air plasma, driven by intense NIR fundamental and second harmonic pulses: A fraction of the NIR pulse is frequency-doubled (SH) in the BBO-crystal and both fields are focused into ambient air. The $\mathrm{THz}$ transient from the airplasma is collimated by a parabolic off-axis mirror. A Brewster-angled silicon wafer filters the $\mathrm{THz}$ radiation. The color photograph displays the experimental implementation.
The AC-bias or two-color scheme for $\mathrm{THz}$ generation in a plasma was first reported in 2000 in Ref. [95], and is based on the mixing of intense fundamental $(800 \mathrm{~nm})$ and weaker second-harmonic pulses in a laser-induced air-plasma, see Figure 3.5. In our implementation, pulse energies up to $2 \mathrm{~mJ}$ are focussed into ambient air to ignite the plasma. Upon focusing, the fundamental beam passes a $100 \mu \mathrm{m}$ thin BBO-crystal for second harmonic generation. The propagation distance from the BBO to the plasma is adjusted to optimize the driving waveform and the $\mathrm{THz}$ generation efficiency. Due to the different refractive indices of air for the fundamental and the second harmonic wave, the resultant optical phases upon superposition in the plasma can be controlled. To a certain degree, this basic optical setup is scalable via increasing the pump power and plasma size due to the absent damage threshold of the nonlinear

medium, and facilitates convenient adjustment of the $\mathrm{THz}$ field strength by a rotation of the BBO angle.

In analogy to the three-step model of high-harmonic-generation [6], the origin of lowfrequency radiation is microscopically explained in a photocurrent model by tunnel ionization and classical electron motion in the laser field [97]. Depending on the phase between the fundamental and second harmonic waves, e.g., for odd integers of $\pi / 2$, a laser field of uneven symmetry is generated which induces a net directional current in the polarization plane. Tunnel current contributions around the laser-field maxima over multiple cycles add up to a macroscopic polarization $P_{M}$ during the pulse envelope, effectively radiating the THz transient $F(t) \propto \partial^{3} / \partial t^{3} P_{M}[90]$.

In a refined model, described in Ref. [98], the ionization and the dynamics of the elec- 
tron wave packet are treated quantum-mechanically via the time-dependent Schrödinger equation. Experimental observations are reproduced, and it is demonstrated that electron collisions with neighboring gas atoms contribute to a coherent buildup of $\mathrm{THz}$ radiation via bremsstrahlung which can result in a delayed additional component in the THz waveform.

In our setup, the energy of the $\mathrm{THz}$ transients is measured with pyroelectric detectors, and the waveform is temporally resolved via electro-optic detection, as described in the following Section 3.5.

\subsection{Electro-optic sampling}

A particular appeal of $\mathrm{THz}$ radiation and the basis of time-domain spectroscopy is the possibility to coherently detect the picosecond electric waveforms. The standard realization employs the Pockels effect, also known as the electro-optic (EO) effect, referring to the linear change of the refractive index $n$ in a nonlinear medium subject to an external electric field.

A detailed description of electro-optic sampling is found in Refs. [99, 100, 101]. In our experimental implementation, THz and 50-fs NIR sampling pulses are co-focused with variable delay into a 500- $\mu \mathrm{m}$ thin (110)-ZnTe EO-crystal, see Figure 3.6e,g. The linear NIR-polarization is rotated by $45^{\circ}$ with respect to the $\mathrm{THz}$ field. During co-propagation in the crystal, the parallel-polarized component of the sampling pulse experiences the THzinduced change in the refractive index, whereas the perpendicular component remains unchanged. The index difference is linear in the THz electric field $\Delta n=n^{3} r_{41} F_{T H z} / 2$ with $r_{41}$ being the effective component of the electro-optic tensor [101]. The induced elliptical NIR-polarization is translated into a polarization rotation via a $45^{\circ}$ quarter-wave plate, and detected as a function of THz-NIR delay by a combination of a Wollaston-prism and two balanced photodiodes. This cross-correlation yields the continuous THz electric waveform.

Principally, 50-fs sampling-pulses support bandwidths up to $20 \mathrm{THz}$, however, a mismatch between the NIR-group velocity and the THz-phase velocity results in a temporal walk-off of the sampled THz-field during co-propagation with the sampling pulse in most EO-crystals and, effectively, in a loss of bandwidth. Furthermore, the maximum bandwidth is limited to $4 \mathrm{THz}$ due to phonon resonances in the experimentally employed $\mathrm{ZnTe}$ crystal [96]. 

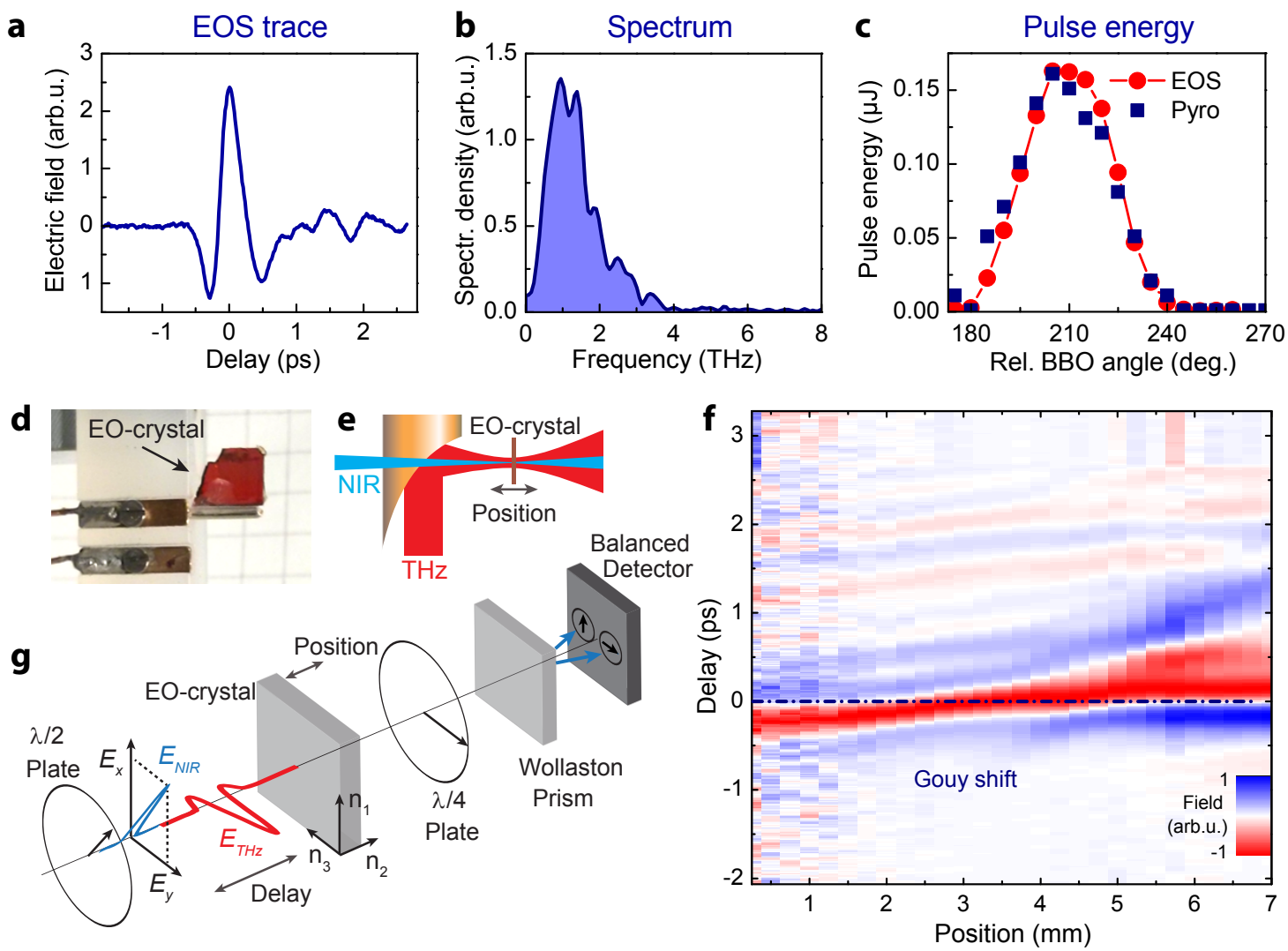

Figure 3.6: a) THz-waveform, detected via electro-optic sampling (EOS) and b) the corresponding spectrum. c) $\mathrm{THz}$ pulse energies are measured as a function of the BBO rotation angle with a pyroelectric detector and plotted with the square of the relative EOS-peak field. d) Color photograph of the experimentally employed ZnTe EO-crystal, mounted onto the sample translation stage inside the vacuum. e) Focusing geometry for EO-sampling. f) Scanning EOS-traces (normalized) along the $\mathrm{THz}$ focus maps the Gouy-phase shift, the reversal of the incident $\mathrm{THz}$ polarity and spectral modulation due to focusing of different spectral components. g) The THztransient $\left(E_{T H z}\right)$ induces an index-change in the EO-crystal, shifting the phase between the two orthogonal polarization components of the sampling pulse $\left(E_{N I R}\right)$. The phase shift is transferred into a polarization-rotation and detected with a polarizing beamsplitter and a balanced detector.

In our experiment, in-situ characterization of the focused THz-waveform at the exact position of the photoemission experiment is achieved by a $4 \times 5 \mathrm{~mm}$ piece of a ZnTe crystal, mounted onto the 3D sample translation stage inside the vacuum chamber (Figure 3.6d). A typical EOS-waveform at the focus position and the corresponding spectrum are given in Figures 3.6a,b. The adjustment of the THz field strength via BBO rotation is characterized via EOS data and pyroelectric pulse energy measurements in Figure 3.6c.

A spatial scan of EOS-traces along the propagation axis in Figure 3.6e,g illustrates a 
fundamental property of any focused beam which is of particular significance for fewcycle transients: Compared to plane wave propagation, the phase velocity in the focus is increased, known as the Gouy phase shift ${ }^{4}$. The on-axis electric field of a Gaussian beam [104] propagating along z-direction is given by

$$
F(z, t)=F_{0} \cdot \frac{w_{0}}{w(z)} \cdot \Re\left\{e^{-i \omega t} \cdot e^{-i\left[k z-\arctan \left(z / z_{r}\right)\right]}\right\}
$$

where $F_{0}=F(z=0, t=0), z_{r}=\pi w_{0}^{2} / \lambda$ denotes the Rayleigh-range and $w(z)$ is the beam waist with the minimum diameter $2 w_{0}$ :

$$
w^{2}(z)=w_{0}^{2}\left[1+\left(\frac{\lambda z}{\pi w_{0}}\right)^{2}\right] .
$$

For Gaussian beams, the arctangent contribution to the phase amounts to a total phaseshift of $\pi$ along the full focal range - implying a polarity reversal for single-cycle pulses, as apparent in Figure 3.6f by the color alternation at zero delay. This focusing feature has significant impact on time-resolved $\mathrm{THz}$ spectroscopy and has to be carefully considered for given focusing conditions.

\subsection{Photoelectron spectroscopy}

\subsubsection{Retarding field energy analyzer}

The earliest implementations of photoemission spectroscopy (PES) reach back to the discovery of photoemission and employ retarding-field energy analyzers [26]. A variable potential barrier forms an electron high-pass filter for the detection of electrons with kinetic energies above the analyzer potential $U_{\text {cutoff }}$. The quantity of transmitted electrons is measured with electrometers or single-electron detectors. The direct energy spectra $N(E)$ are obtained via differentiation of the recorded cumulated energy distributions $\int_{U_{\text {cutoff }}}^{\infty} N(E) d E$ with respect to $U_{\text {cutoff }}$, introducing sensitivity to fluctuations in the total electron yield and often necessitating noise-reduction by averaging or data pre-processing. Advanced, more sensitive and high-resolution analyzers are realized as band-pass filters based on kinetic energy-dependent trajectories in suitably-shaped static electric fields [30,

\footnotetext{
${ }^{4}$ An intuitive explanation considers geometrical properties of the Gaussian beam [102]. A more general derivation follows from the spatial confinement of transverse momenta and the uncertainty principle [103].
} 
pp. 20-23].

The retarding-field analyzers, employed in the measurements of Chapter 4 and Chapter 5, Figure 5.2, consist of four parallel gold-coated molybdenum grids to filter and reaccelerate the electrons towards the detector. The transmitted electron yield is amplified by $10^{6}$ with a double-stacked micro-channel-plate (MCP) and detected with singleelectron sensitivity either as a voltage pulse at a collection anode or as a spatially-resolved optical signal on a phosphor-screen with a CCD camera.

\subsubsection{Time-of-flight electron spectrometer}

The second experimental approach employs an electron time-of-flight (TOF) spectrometer, capable of high-throughput, single-shot spectral acquisition. Triggered by laserinduced photoemission, a fast analog-digital converter registers the electron time-of-flight from the sample to the fast MCP detector with nanosecond-resolution. The electron energy distribution temporally disperses along the drift-path $s_{d r i f t}$, thus translating kinetic energy $E$ into time:

$$
t=\sqrt{s_{d r i f t}^{2} m_{e} / 2 E}
$$

Recorded arrival time histograms $N(t)$ are converted to electron spectra $N(E)$ by the variable transformation $t \rightarrow E$, introducing the multiplicative weighting term:

$$
N(E)=N(t)\left|\frac{d t}{d E}\right|=N(t) \sqrt{\frac{s_{d r i f t}^{2} m_{e}}{8}} E^{-3 / 2} .
$$

The experimentally employed spectrometer (ETF11, Stefan Kaesdorf, Germany) [105] collects and collimates electrons from a working-distance of $3 \mathrm{~mm}$ within an acceptance angle of $45^{\circ}$ by an extraction module with an electrostatic electron lens of adjustable potential, see Figure 3.7a. After traversing a field-free drift length of $294 \mathrm{~mm}$, the electrons are accelerated to $1000 \mathrm{eV}$ in a post-acceleration stage onto the double-plate MCP detector. The MCP response is recorded with a digital oscilloscope supporting $1 \mathrm{GHz}$ analog bandwidth and a sampling rate of $5 \mathrm{GSa} / \mathrm{s}$ (Agilent infinium DSO9104H, Agilent, USA). The $1 \mathrm{kHz}$ trigger signal is derived from a reflected part of the NIR excitation beam on a Si-photodiode (ET 2030, Electro-Optics Technology, Inc., USA) with a rise time below 300 ps. A constant time-shift between the trigger signal and the instant of photoemission ('time zero') has to be determined for time-to-energy calibration. Moreover, the actual 

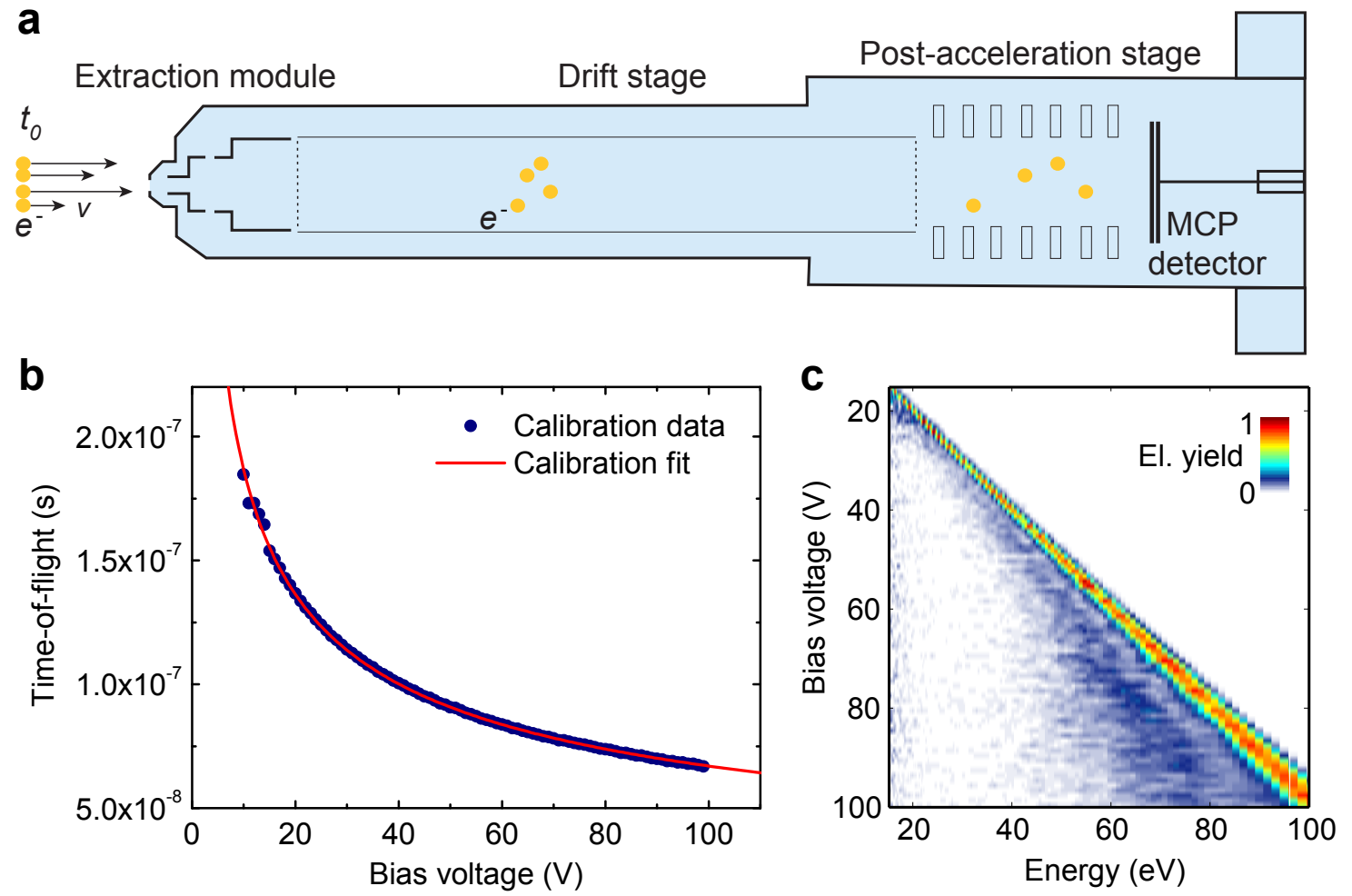

Figure 3.7: Time-of-flight electron spectrometer (TOF). a) Schematic of the TOF, displaying the extraction module which collimates the electrons, the drift stage where electrons temporally disperse and the post-acceleration and detection unit. b) Experimental flight-times for varying bias voltages for TOF calibration at a lens voltage of $800 \mathrm{~V}$. b) The representation of the calibration spectra for varying bias voltage displays the decreasing spectral resolution at higher energies and a lower-energy pedestal. Notably, this acquisition mode allows for broadband single-shot detection of kinetic energy distributions from $15 \mathrm{eV}$ to above $100 \mathrm{eV}$.

spectrometer design departs from an ideal field-free drift-path and a resultant, more complex electron motion has to be considered. The relevant parameters are obtained by a calibration scheme, employing the variable bias potential of the metallic sample to record emission spectra for various predefined electron kinetic energies, see Figure 3.7b. From the known kinetic energies, the corresponding pure flight-times, effective drift lengths and the time zero are extracted for different acquisition modes. The nonlinearity between the time-of-flight $t_{T O F}$ and $E$ implies a higher resolution at low kinetic energies, for instance, varying between $\Delta E=200 \mathrm{meV}$ for $E_{k i n}=10 \mathrm{eV}$ and $\Delta E=7 \mathrm{eV}$ for $E_{k i n}=100 \mathrm{eV}$ at spectrometer settings that allow for the single-shot acquisition of such broadband spectra, see Figure 3.7c. This experimentally employed, above- $100 \mathrm{eV}$ broadband acquisition mode represents a rather unconventional operation, and higher energy resolution for fast 


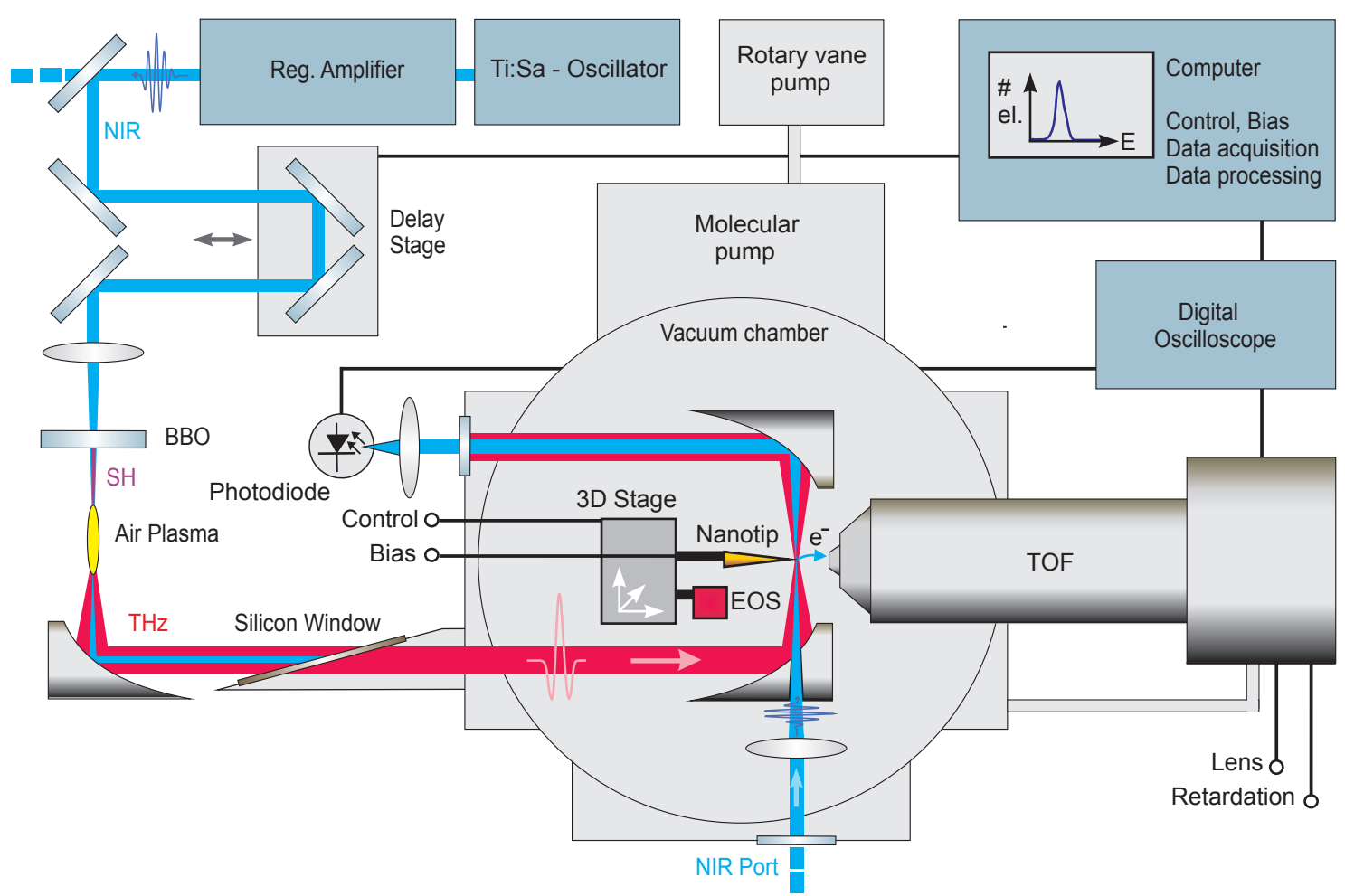

Figure 3.8: Sketch of the Terahertz setup. Time-delayed THz transients are generated in a twocolor air plasma, coupled into the vacuum chamber and co-focused with NIR pulses. A nanotip or an electro-optic sampling crystal (EOS) can be transferred into the focus. Photoemission is detected and energetically resolved with a time-of-flight electron spectrometer (TOF), triggered via the fundamental beam and a fast photodiode.

electrons is typically achieved by the application of a decelerating potential prior to the drift-stage.

\subsection{Optical setup and experimental chamber}

The experimental setup for the mid-infrared experiments is sketched in Supplement Figure 4.5. Infrared beam routing from the source to the sample is facilitated via gold and silver mirrors with negligible absorption due to the broadband metallic reflectivity. Focusing in absence of chromatic aberations is achieved via $90^{\circ}$-off-axis parabolic gold mirrors with a wavelength-independent focal length of $25 \mathrm{~mm}$ (Edmund Optics $\mathrm{GmbH}$, Germany).

A pair of infrared wire-grid polarizers (Tydex J.S. Co., St. Petersburg, Russia) is employed for beam attenuation for a wavelength range of $1.5-20 \mu \mathrm{m}$. Transmission is min- 
imized for a polarization parallel to the aluminum coated grooves and maximized for a vertical polarization up to $70 \%$, achieved by a broadband anti-reflective coating at the back side of the high refractive zinc-selenide ( $\mathrm{ZnSe}$ ) substrate.

Optical windows made from $\mathrm{ZnSe}$ feature a flat transmission and low dispersion from $500 \mathrm{~nm}$ up to $19 \mu \mathrm{m}$. They are used to optically couple the laser pulses into the experimental ultra-high-vacuum (UHV) chamber. A base pressure at $10^{-8} \mathrm{mbar}$ is achieved via a turbo-molecular pump combined with a scroll pump.

The Terahertz setup used for the experiments in Chapters 5,6, is implemented as a pump-probe-scheme, see Figure 3.8, combining the 800-nm fundamental beam and the time-delayed $\mathrm{THz}$ transient at the sample position. The $\mathrm{THz}$ transient is focussed via an off-axis parabolic gold mirror ( $f_{f o c}=25 \mathrm{~mm}$, Edmund Optics GmbH, Germany) with a custom-drilled hole for the combination with the lens-focussed NIR beam $\left(f_{f o c}=100 \mathrm{~mm}\right)$. An electro-optic crystal, mounted on the sample translation stage, is employed to characterize the incident THz-waveform in the focus (see Chapter 3.5). The vacuum setup is equivalent to the mid-infrared experiment, but using a Brewster-angled silicon wafer as an optical port for the THz radiation and optical windows for the NIR-beam.

\subsection{Nanoscopically-sharp gold and tungsten needles}

The confinement of light below the diffraction limit and high local intensity enhancement are among the most outstanding optical properties of metallic nanostructures. Different from free-propagating light waves, in which the energy oscillates between electric and magnetic fields, at metallic nanostructures a substantial fraction of the energy can alternate between collective oscillations of quasi-free conduction band electrons, and the electric and magnetic fields. The excitations of the charge density oscillations are termed plasmons, and depending on the optical excitation conditions, the material properties and the respective geometry, distinct forms can be resonantly excited. At a metal-dielectric interface, surface plasmon polaritons (SPP) can propagate along the interface and induce electric near-fields which exponentially decay into the dielectric and into the metal. At planar surfaces, SPPs are not directly excited and boundary conditions require for $\varepsilon_{m}$, the complex dielectric constant of the metal, $\mathfrak{R}\left(\varepsilon_{m}\right)<0$ and $\mathfrak{R}\left(\varepsilon_{m}\right)>\mathfrak{I}\left(\varepsilon_{m}\right)$. These conditions are satisfied at gold-vacuum interfaces in the optical spectrum at $\lambda=633 \mathrm{~nm}$ [106]. For confined particles, the excitation conditions and the effective dielectric function are modified. For example, localized surface plasmon polaritons (LSPP) are bound to nanos- 
a

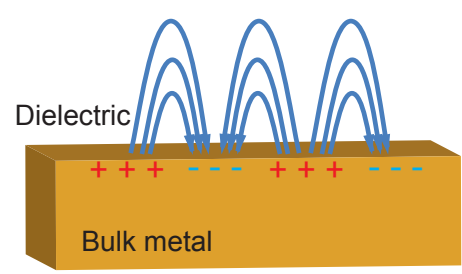

b

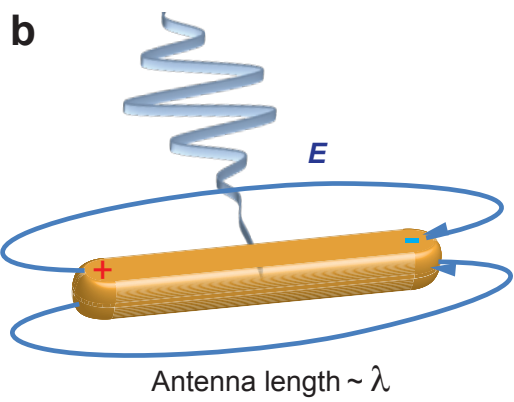

C

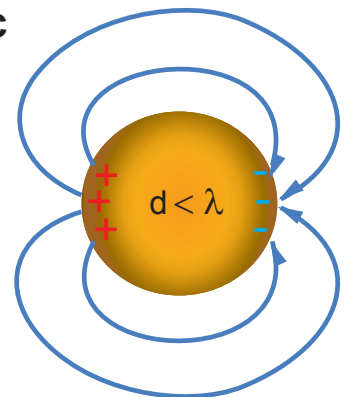

Figure 3.9: Near-field enhancement and confinement of optical fields are achieved with plasmonic resonances, a) at metal-dielectric interfaces due to surface plasmon polariton (SPP) excitations, b) at nanoantennas with lengths resonantly matched to the driving wavelength, or (c) at sub-wavelength nanoparticles via resonant excitation of localized surface plasmon polaritons (LSPP).

tructures with sub-wavelength dimensions and can directly be excited by plane wave illumination. In resonant nanoantennas with lengths close to the driving wavelength (e.g., half-wave antennas), plasmonic modes are excited as oscillating standing charge waves along the structure, and charges are accumulated at the ends, as illustrated in Figure 3.9. Further examples and a more detailed description are found in Refs. [106, 107].

The experimentally employed nanostructures are metallic nanotips of polycrystalline gold and tungsten. At sharp gold tips with few-nm apex radii, high local field enhancements due to plasmonic resonances can be directly excited at NIR-wavelengths [108, 109, 110]. In a simplified analogy, the driven charge oscillation at the apex is compared to an oscillating vertical dipole in the center of a spherical charge distribution [109]. The respective electric near-field distribution is sketched in Fig.3.10a and a corresponding emission pattern is experimentally observed in Ref. [109]. A dipolar near-field distribution typically decays with the distance as $r^{-3}$ from the apex. The numerical solution of the Maxwell equations yields standing surface plasmon waves with the maximum charge accumulation at the tip apex [108]. The experiments in Ref. [111] indicate that such resonances are highly sensitive to the exact geometry of the tip, and that - depending on the shape - the resonance may vary from optical to near-infrared frequencies or even vanish. At tungsten nanotips, similar resonant excitations are not observed, resulting from the positive real part of the dielectric function, $\Re\left(\varepsilon_{m}\right)>0$, at these wavelength, and the large imaginary part, $\mathfrak{I}\left(\varepsilon_{m}\right)>\mathfrak{R}\left(\varepsilon_{m}\right)$.

A non-resonant mechanism providing field enhancement at the nanotip apex is the con- 

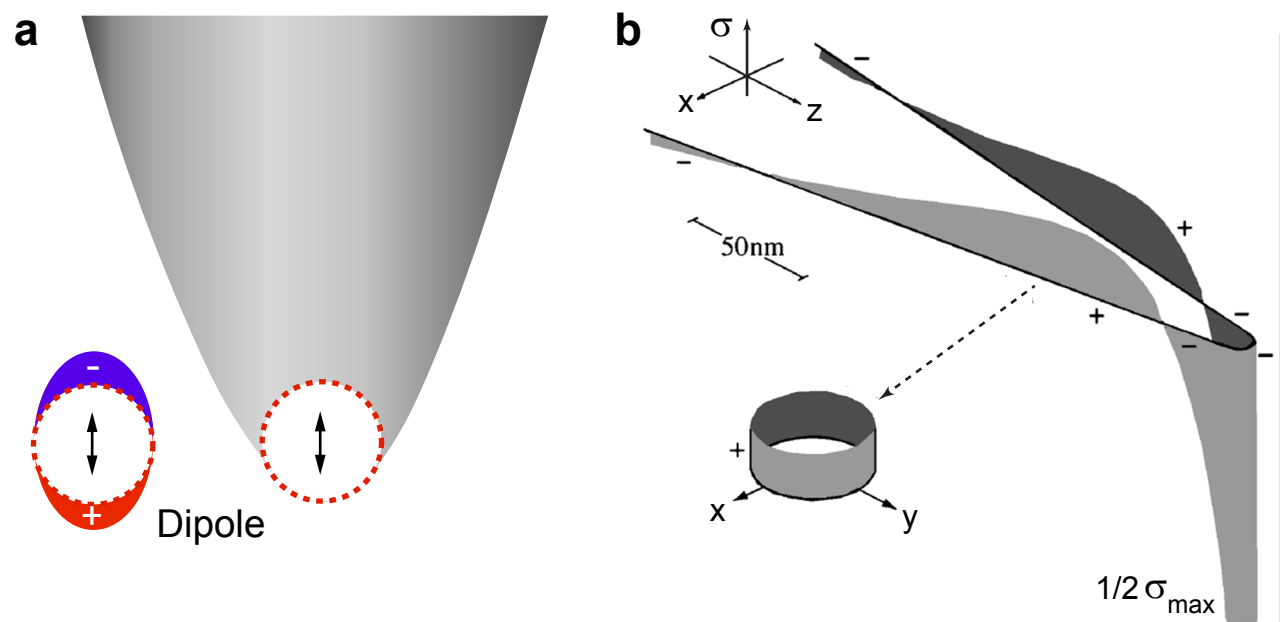

Figure 3.10: a) Enhanced near-field at a nanotip, modeled as a vertically oscillating dipole in the tip apex. The colors (blue, red) illustrate the near-field distribution. b) Numerical calculation of the induced surface charge density $\sigma$ of a gold tip, illuminated at a wavelength of $\lambda=830 \mathrm{~nm}$ and polarization parallel to the tip axis (z-coordinate). The solution of the Maxwell equation yields standing charge-density waves and demonstrate the charge accumulation at the apex, resulting in the near-field enhancement. Figure adapted from Ref. [108].

centration of electric field lines due to the sharp geometry of the metallic conductor and the resulting accumulation of surface charge, also known as the lightning rod-effect. At NIR-wavelengths, this "geometric" field enhancement factor exceeds values of 5 for nanotips from gold and tungsten with apex radii of $10 \mathrm{~nm}$ and smaller [112].

The static electric field distribution is analytically solvable for paraboloidal and hyperboloidal tip geometries [46]. We evaluated the paraboloidal solution in the form of Ref. [113] for a $100 \mathrm{~V}$ potential at a tip of $25 \mathrm{~nm}$ apex radius, shown in Figure 3.11e. For the similar hyperboloidal case, the field along the tip axis can be described as:

$$
F(z)=\frac{F_{0}}{1+\frac{2}{r_{0}} r} \text {. }
$$

On the scale of the apex radius $r_{0}$, the near-field decays to $1 / 3$ of the surface field $F_{0}$.

Notably, the long-range $r^{-1}$-decay of the field results - for static acceleration of electrons from the tip apex - in the acquisition of only 35\% of the potential energy upon propagation over a distance of hundred apex radii $\left(100 \cdot r_{0}\right)$ [46]. The difference to $99 \%$ potential energy for the acceleration from a charged sphere, which decays with $z^{-3}$, is caused by the long-range electrostatic effect of the conical shaft. 

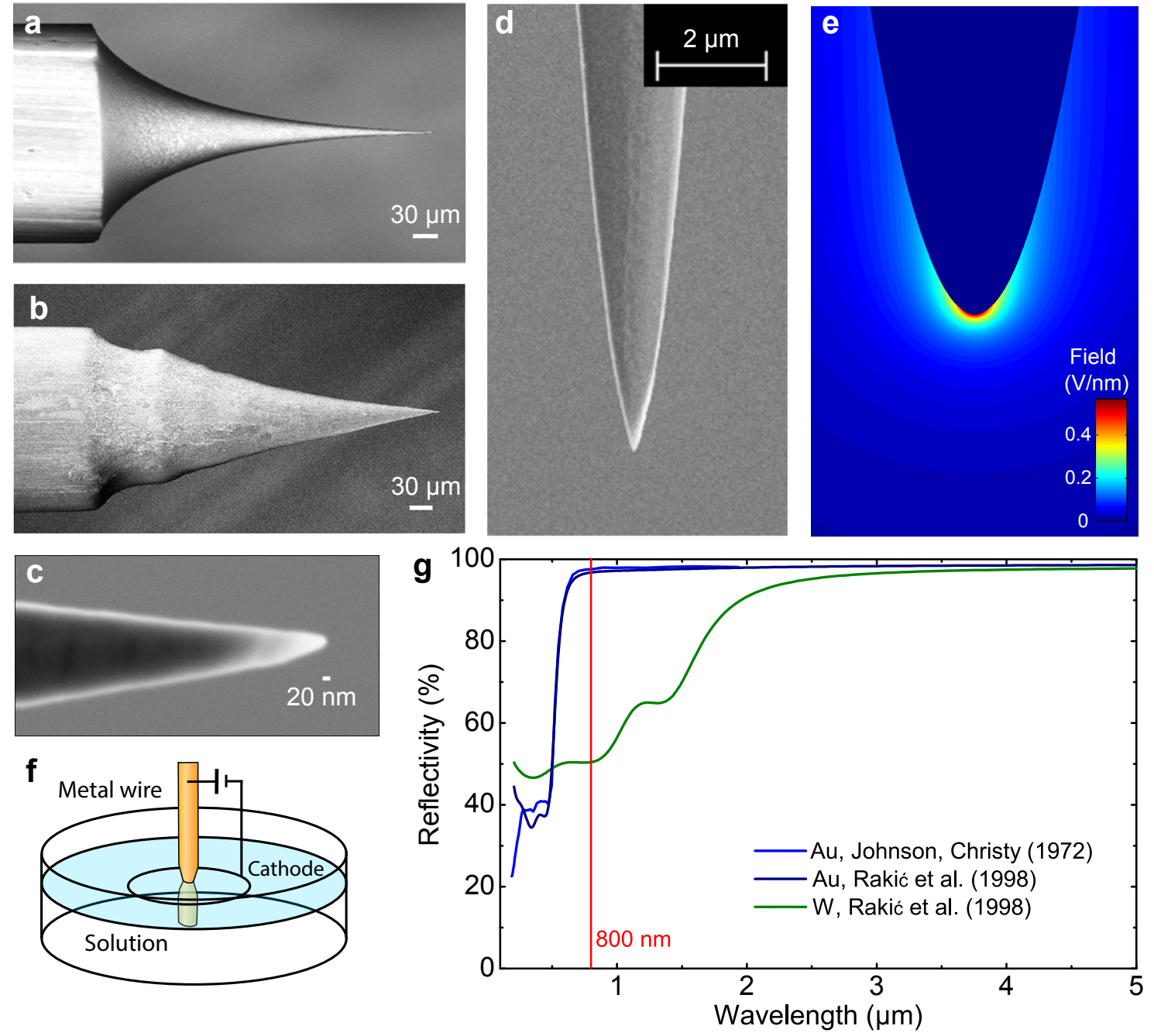

Figure 3.11: Scanning electron micrographs of tungsten (a) and gold (b) nanotips display characteristic shaft geometries due to the respective etching process. c) The experimentally employed nanotips exhibit apex diameters of 10-20 nm and particularly smooth surfaces (d) extending over $20-50 \mu \mathrm{m}$ from the apex. e) Electrostatic field distribution for a paraboloidal tip, calculated according to Ref. [46] for a $25 \mathrm{~nm}$ tip radius at a potential of 100 Volts. f) Sketch of the electro-chemical wet-etching setup. Due to the enhanced etching rate in the meniscus region, the lower part of metal wire ultimately ruptures and leaves the sharpened upper tip geometry. g) Reflectivity for perpendicular incidence on gold and tungsten surfaces. The $800 \mathrm{~nm}$ wavelength of NIR-excitation is marked in red. Optical constants from Refs. [114, 115, 116].

The nanotips are fabricated by electro-chemical wet-etching of a gold wire with $250 \mu \mathrm{m}$ diameter in an HCl-ethanol-solution [117] or tungsten wire of 100 and $250 \mu \mathrm{m}$ diameter in $\mathrm{NaOH}$ [118], as sketched in Figure 3.11f. Apex diameters of 10-20 nm are achieved, char- 
acterized by scanning-electron microscopy (SEM), see Figure 3.11c. The typical shaft profiles are determined by the etching process and display a concave curvature for tungsten tips and a mostly linear conical geometry for gold tips, as shown in Figures 3.11a,b, respectively. For the experiments, structures with sharp apex geometries and smooth surfaces, extending over the illumination region without significant surface defects, protrusions and roughness, are selected.

The high reflectivity for both metals at long wavelengths is shown in Figure 3.11b, reaching above $95 \%$ for mid-infrared wavelengths above $3 \mu \mathrm{m}$ and extending to the farinfrared. The NIR-reflectivity of $50 \%$ for tungsten at $800 \mathrm{~nm}$ wavelength is substantially lower compared to gold (97\%), but the material features a high melting point of $3695{ }^{\circ} \mathrm{C}$ $\left(1337{ }^{\circ} \mathrm{C}\right.$ for gold) and superior mechanical stability. Additionally, the electron-phonon coupling constant of tungsten $g_{W}=2 \cdot 10^{17} \mathrm{Wm}^{-3} \mathrm{~K}^{-1}$ is an order of magnitude higher than that of gold $g_{A u}=2.2-4 \cdot 10^{16} \mathrm{Wm}^{-3} \mathrm{~K}^{-1}$, allowing for a rapid decay of laser-excited hot-electron populations [119].

\subsection{Streaking spectroscopy}

The electronic acquisition of ultrashort optical signals is limited by detector bandwidths to timescales of several picoseconds. A versatile concept to capture much faster optical transients employs the projection of temporal information onto a different physical observable $^{5}$. In this way, the requirements for temporal discrimination are transferred to the projection and to the corresponding (non-temporal) resolution of a slow detector. Compared to temporal gating via optical nonlinearities, which represents a different method for ultrafast temporal resolution, this concept is also well-suited for the characterization of transients at low-intensity and facilitates the current frontiers of ultrafast science [4, 123].

In streak-cameras, a widely-employed implementation of electro-optic streaking, the ultrashort optical signal triggers the emission of electrons from a photocathode into a cathode ray tube [124, p. 259-261]. Upon propagation through a rapidly swept electric field, the electrons are accelerated perpendicularly and deflected or "streaked" to different positions on the detector screen, depending on their emission time. Thus, initial photon

\footnotetext{
${ }^{5}$ TOF-spectrometers project time onto "stretched" time via the dispersion of electron propagation. Alternatively, time can be spectrally encoded [120] and the dispersion of long optical fibers ("Dispersive Fourier Transform") can be used to all-optically stretch ultrafast signals for the single-shot detection with comparatively slow photodiodes. For the latter see Ref. [121] and our recent application in Ref. [122].
} 
numbers and arrival times at the photocathode are encoded onto a streaking trace, which is recorded by a spatially resolving electron detector. Commercial, state-of-the-art designs allow for a temporal resolution around $100 \mathrm{fs}$ [125].

\subsubsection{All-optical streaking}

Ultimate temporal resolution is achieved with attosecond streak cameras, employing alloptical streaking concepts [4]. In a typical realization, electrons are photo-ionized from a noble gas by sub-fs UV pulses, transferring phase and amplitude information onto electron wave packets [126]. The latter are analyzed by the time-dependent acceleration in the electric field of a second streaking pulse, oscillating at optical periods $T$ comparable to the UV-pulse duration $t_{p}$. The electric laser field $F_{\text {streak }}(t)$ acts in a temporally integrating fashion on the electrons, starting at the instant of emission $t_{e}$, and imprints the streaking vector potential $A\left(t_{e}\right)$ onto the electron kinetic energy [4],

$$
A\left(t_{e}\right)=\int_{t_{e}}^{\infty} F_{\text {streak }}\left(t^{\prime}\right) d t^{\prime}
$$

As the final kinetic energy depends on the emission time, the UV amplitude and the phase are encoded onto the electron yield and the streaking energy, respectively. A continuous temporal evolution is mapped by recording electron energy spectra as a function of the delay between emission and streaking pulses. For example, the streaking vector potential of few-cycle light waves at $400 \mathrm{THz}(800 \mathrm{~nm})$ is phase-resolved via 250 -as XUV photoemission [127] (see Figure 3.12a), yielding the transient electric field via differentiation. Conversely, the scheme enables the characterization of the ionizing attosecond transients, e.g., evidencing the generation of isolated 80 attosecond XUV pulses [2].

The temporal resolution of all-optical streaking is governed by the slope of the streaking field, representing the leverage of the projection from time to kinetic energy. A signal is typically streaked in the linear slope of the streaking field oscillation, which corresponds to a quarter of the streaking period $T_{\text {streak }}$. As the temporal resolution is increased with the frequency of the streaking field, the temporal duration of the detection window $T_{\text {streak }} / 4$ decreases. Streaking at $800 \mathrm{~nm}$ wavelength, for example, results in a temporal detection window below 700 as. Thus, all-optical streaking of signals with durations of several tens of fs requires the adaptation to few- $\mathrm{THz}$ streaking frequencies, as experimentally implemented for the single-shot characterization of X-ray pulses from the FLASH free- 

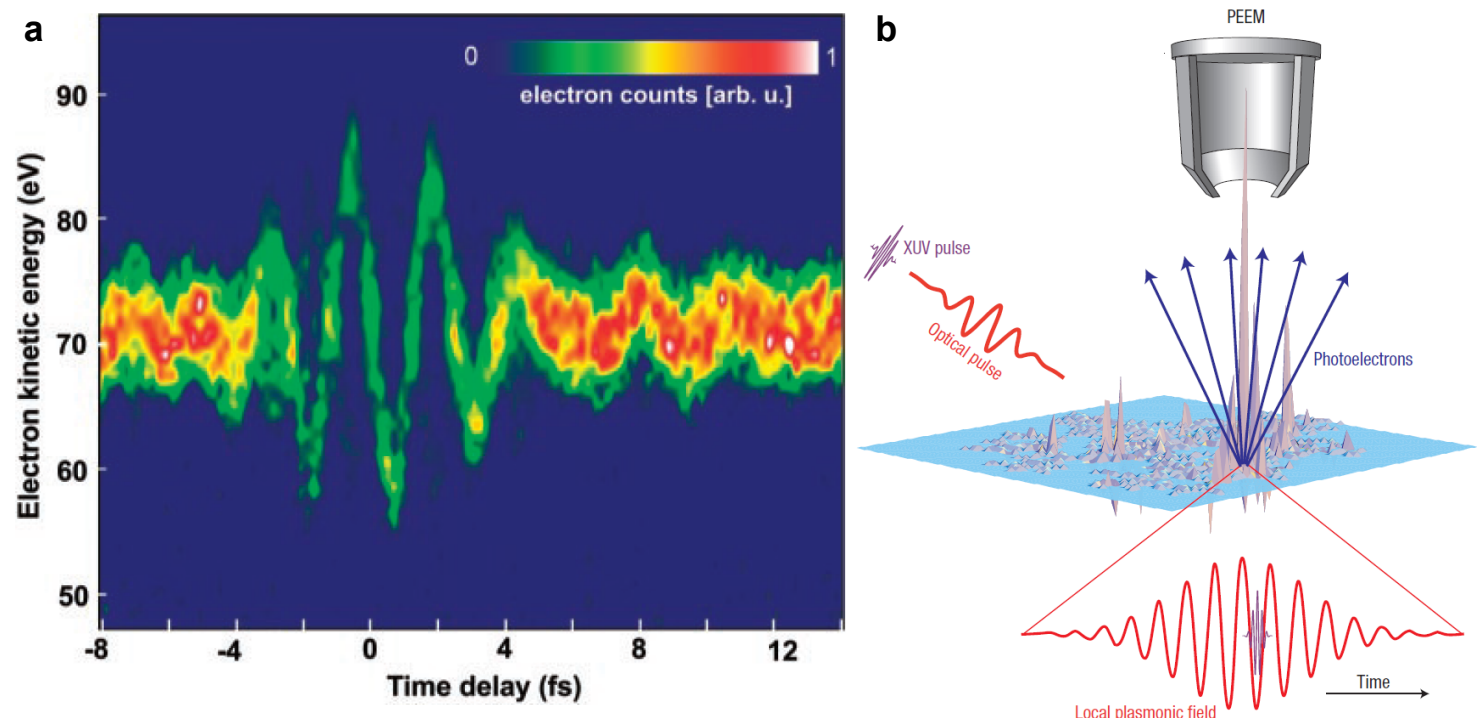

Figure 3.12: a) Experimental streaking spectrogram, resolving the vector-potential of a few-cycle $800 \mathrm{~nm}$ streaking field. Photo-ionization is induced from Neon via 250-as XUV photoemission at $93 \mathrm{eV}$ photon energy. Image from Ref. [127]. b) Illustration of the proposed attosecond microscope for the temporally- and spatially-resolved detection of photoelectrons in plasmonic near-fields. Figure from Ref. [128].

electron laser or table-top femtosecond XUV sources [129, 130].

\subsubsection{Nanoplasmonic streaking}

Transferring optical streaking concepts to nanostructures offers the prospects of phaseresolved detection of plasmonic near-fields and temporal mapping of carrier dynamics at the surface [131] and inside of nanostructures, as described in Chapter 6.

Attosecond streaking at nanostructures was proposed in Ref. [128] as a combination of all-optical streaking with photoemission electron microscopy (PEEM) to add nanoscopic spatial selectivity. The study, along with extensive theoretical work on streaking in less complex setups at isolated nanoparticles [132, 133, 134, 135], elaborates the impact of the near-field localization onto the streaking process. Contrary to far-field streaking in macroscopic foci, highly energetic UV-photoelectrons can exit the localized plasmonic streaking-field instantaneously - resulting in a transfer of electron momenta in-phase with the streaking near-field.

The experimental implementation of nanoplasmonic streaking with UV-photoemission is being pursued by several groups, but remains highly challenging due to photoemission 
contributions from the macroscopic extension of the illuminated structure, as reported, e.g., in Ref. [136]. In the present work, we utilize the localization of NIR-photoemission to the apex of a single nanotip, and implement near-field streaking at Terahertz frequencies. 


\title{
Chapter 4
}

\section{Field-driven photoemission from nanostructures quenches the quiver motion}

\author{
G. Herink, D. R. Solli, M. Gulde and C. Ropers
}

Nature 483, pp.190-193, March 2012

DOI: $10.1038 /$ nature 10878

Strong-field physics, an extreme limit of light-matter interaction $[6,7,36]$, is expanding into the realm of surfaces $[115,137]$ and nanostructures $[60,63,64,66,70,138]$ from its origin in atomic and molecular science $[4,56,139,140]$. The attraction of nanostructures lies in two intimately connected features: local intensity enhancement and sub-wavelength confinement of optical fields. Local intensity enhancement facilitates access to the strongfield regime and has already sparked various applications, whereas spatial localization has the potential to generate strong-field dynamics exclusive to nanostructures. However, the observation of features unattainable in gaseous media is challenged by manybody effects and material damage, which arise under intense illumination of dense systems [141, 142, 143, 144]. Here, we nondestructively access this regime in the solid state by employing single plasmonic nanotips and few-cycle mid-infrared pulses, making use of the wavelength-dependence of the interaction, that is, the ponderomotive energy. We investigate strong-field photoelectron emission and acceleration from single nanostructures over a broad spectral range, and find kinetic energies of hundreds of electronvolts. We observe the transition to a new regime in strong-field dynamics, in which the electrons escape the nanolocalized field within a fraction of an optical half-cycle. The transition into this regime, characterized by a spatial adiabaticity parameter, would require relativistic electrons in the absence of nanostructures. These results establish new 


\section{degrees of freedom for the manipulation and control of electron dynamics on fem- tosecond and attosecond timescales, combining optical near-fields and nanoscopic sources.}

The explanation of the photoelectric effect by Einstein in 1905 marks a defining moment in the turn towards modern physics, reconciling numerous observations contradicting classical expectations [29]. Einstein's contemporaries had been puzzled by the scaling of photoelectron kinetic energies: increasing with light frequency and independent of light intensity. Some quotations from Lenard's 1902 experimental paper illustrate the historical confusion. He expected that "the part of the initial velocity stemming from the light...should have been acquired within the last half or full resonance oscillation, hence it ought to grow with light intensity," but in experiments found "not the slightest dependency...on light intensity" [27]. Here we show that nanostructures exposed to intense mid-infrared fields may indeed cause classical electron dynamics determined by less than an optical half-cycle.

Today, it is known that strong-field interactions of light with atoms and surfaces, particularly electron emission, are governed by both quantum-mechanical and classical concepts, given that the final state of a photoemission event is influenced by the presence of the optical field [36]. The underlying principles have been widely studied in atoms and molecules $[4,6,7,56,139,140,145]$ and classical scalings become increasingly pronounced at higher intensity and longer wavelength $[7,56]$. The photon-driven (quantum) and the field-driven (classical) regimes are regarded as limiting cases [36] with a transition characterized by the Keldysh parameter $\gamma=\omega / \omega_{t}$. Also known as the adiabaticity parameter, it relates the optical driving frequency $\omega$ to a characteristic tunnelling frequency $\omega_{t}=e F / \sqrt{2 m \Phi}$ (where $F$ is the electric field, $\Phi$ is the work function, $m$ is the mass of the electron and $e$ is its charge). In the strong-field/low-frequency limit $(\gamma<<1)$, the emission is viewed as tunnelling that adiabatically follows the momentary optical field. The energies of photoelectrons are then determined by their quiver motion in the driving field $[6,7]$ and their energy cutoffs scale with the ponderomotive potential $U_{p}=e^{2} F^{2} / 4 m \omega^{2}$. These two steps, adiabatic tunnelling and propagation of classical point particles, form the basis of the so-called Simpleman model $[6,7]$, which accurately describes numerous aspects of strong-field atomic ionization.

The standard Simpleman description of electron trajectories contains only the temporal - not the spatial - dependence of the driving field because the wavelengths or typical 

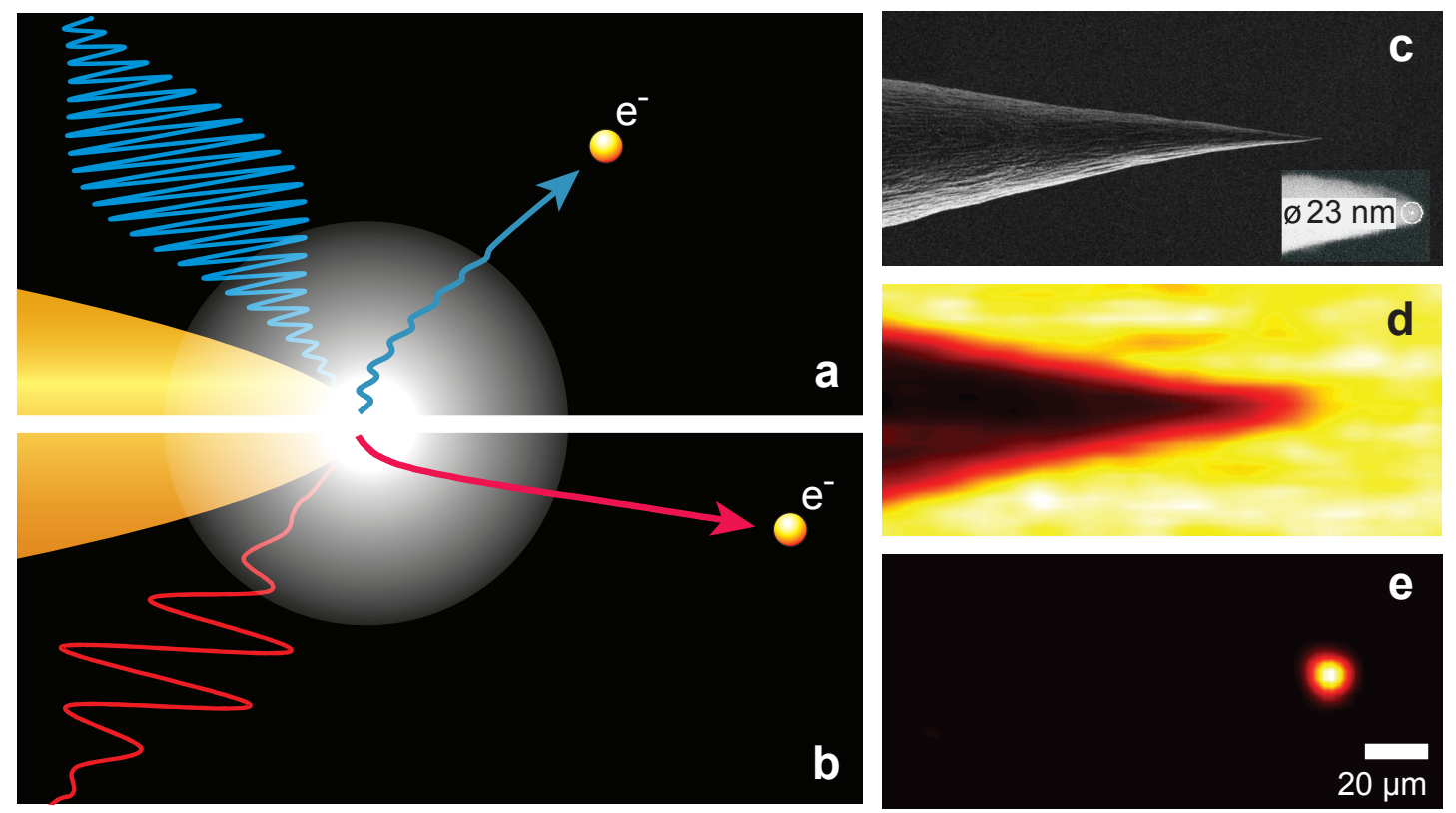

Figure 4.1: Electron emission from nanotips using ultrashort mid-infrared fields. Trajectories of photoelectrons generated by intense light fields depend strongly on whether the quiver amplitude is smaller (a, short-wavelength excitation) or larger (b), long-wavelength excitation) than the characteristic decay length of the optical near-field (bright white region). In $b$, the electron escapes the near-field before substantial back acceleration. c, Scanning electron micrograph of gold tip used in measurements. Inset, apex region and tip diameter. $d$ and e, Mid-infrared shadow image (d) and electron-emission image (e), obtained by scanning the tip through the focal plane, demonstrating exclusive electron emission from the tip apex (wavelength $3.8 \mu \mathrm{m}$ ). We note that the spot size in e represents the focus diameter, while the actual emission region has nanometre dimensions.

optical focus diameters are usually much larger than the quiver amplitude. Simple considerations arising from the diffraction limit show that this condition generally holds for non-relativistic energies. In contrast, the optical fields at nanostructures may vary greatly on such scales, so that entirely different electron dynamics, which have not yet been observed, may be expected (see sketches in Fig. 4.1a, b). Recently, numerous studies have addressed localized emission of electrons from nanometric metallic needles using 800$\mathrm{nm}$ excitation radiation $[53,57,60,63,70,146]$. The possibilities for local enhancement and control of optical fields make strongfield physics in nanostructures highly attractive [60, 63, 64, 66, 70, 128, 132, 138]. However, many-body effects complicate matters, and short-wavelength damage thresholds limit Keldysh parameters close to unity, with ponderomotive acceleration just arising with energies around $10 \mathrm{eV}$. Higher energies have 
been achieved in plasmonic fields at planar surfaces [115, 137], but interpretations can be complicated by inhomogeneous broadenings from surface roughness and the inclusion of space charge [141, 142, 143, 144].

Here, we study ultrafast strong-field photoemission from single nanostructures over a wide range of wavelengths up to $8 \mu \mathrm{m}$, reaching deep into the tunnelling regime, with Keldysh parameters as low as 0.1 . We identify wavelength scalings of classical origin that differ substantially from usual ponderomotive behaviour. This follows directly from particle dynamics in fields decaying over distances shorter than the quiver amplitude. In particular, we observe a transition to a new subcycle regime, in which the electrons' kinetic energy, reaching hundreds of electronvolts, becomes largely wavelength-independent. This transition is characterized by introducing a spatial complement to the Keldysh parameter.

In our experiments, tunable femtosecond pulses are focused onto electrochemically etched gold tips (radius of curvature about $10 \mathrm{~nm}$; Fig. 4.1c) in ultrahigh vacuum. The kinetic energy spectra of photoelectrons are recorded as a function of intensity across the near- and midinfrared spectrum. Figure $4.1 \mathrm{~d}$ and e illustrates the confinement of the photoemission to the apex (wavelength $3.8 \mu \mathrm{m}$ ) with simultaneous measurements of the light passing the tip (Fig. 4.1d) and the induced electron emission (Fig. 4.1e) from scanning the tip in the focal plane.We find such apex-exclusive signals at all wavelengths, evidencing the ultra-broadband nature of both field enhancements (arising from geometry and plasmonic resonance) and nonlinear photoemission at these tips [57, 109, 147]. Electron spectra (Fig. 4.2a) from two tips of different radii and at various intensities show the scaling of kinetic energies up to cutoffs of hundreds of electronvolts.

To examine the observed behaviour, we compute electron spectra using experimental parameters in a two-step model adapted to localized fields. The simulations (Fig. 4.2a, solid lines) include a Fowler-Nordheim tunnelling model (justified, for example, in ref. [61]), interaction of the electrons with a strongly localized tip field and rescattering at the surface (see Methods Summary). Good agreement with the experimental data is obtained. Throughout this work, we use the local (that is, tip-enhanced) fields in expressions and values of the Keldysh parameter $\gamma_{l o c}$. For example, the curve extending to $300 \mathrm{eV}$ in Fig. $4.2 \mathrm{a}\left(\gamma_{l o c}=0.13\right)$ corresponds to a local peak field strength of $28 \mathrm{~V} \mathrm{~nm}^{-1}$. The two tips of different radii exhibit different total currents and cutoffs at the same ( $\gamma_{l o c}=0.16$ ) for different emission areas (influencing the total current) and field decay lengths. As we discuss later, tighter confinement in fact leads to a cutoff reduction.

At such high energies and significant electron densities, the influence of many-body 

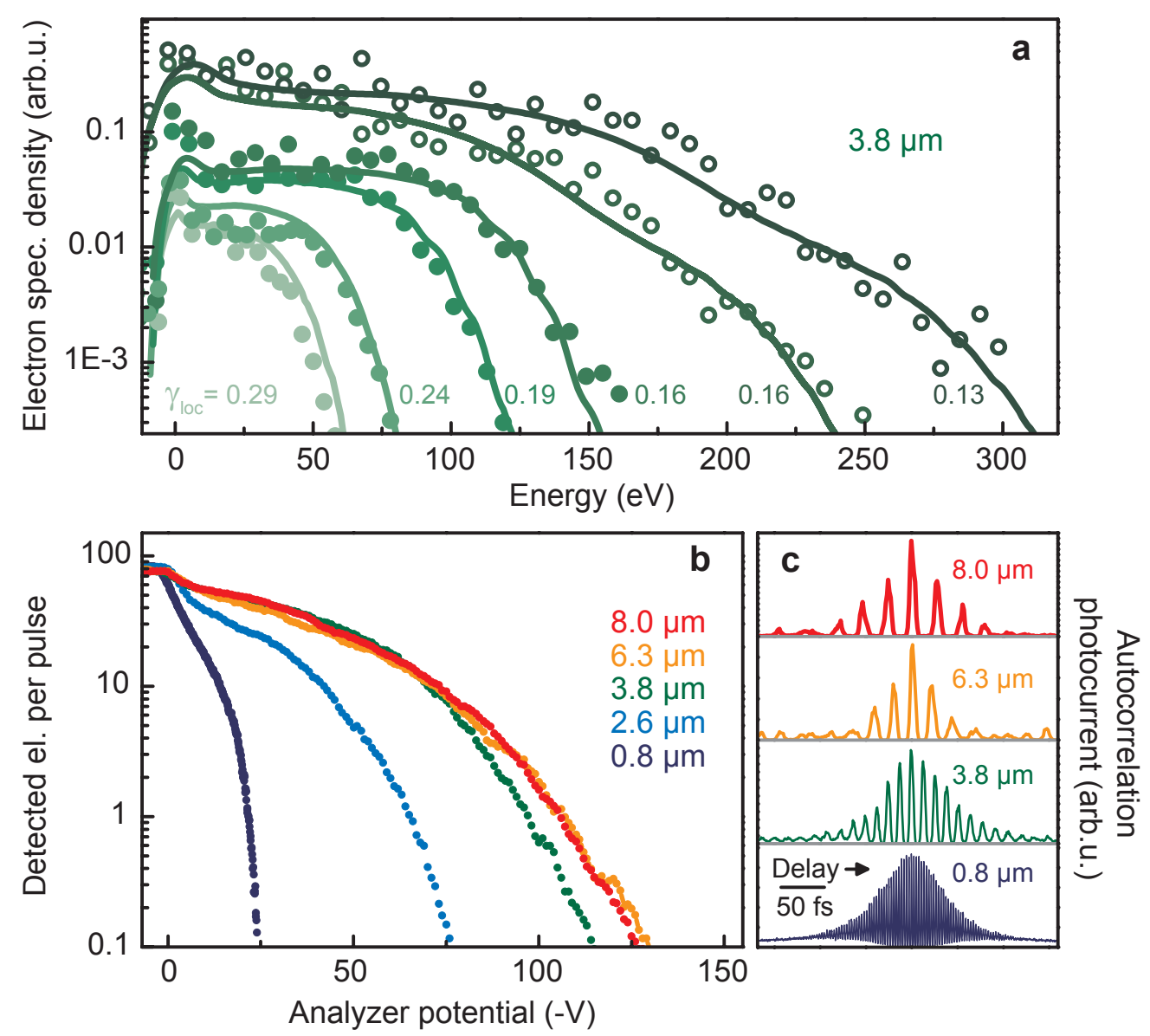

Figure 4.2: Experimental photoelectron spectra and autocorrelations at midinfrared wavelengths. a, Experimental (circles) and simulated (solid lines) kinetic energy distributions of photoelectrons for increasing intensities at wavelength $3.8 \mu \mathrm{m}$. Tip radii are $12 \mathrm{~nm}$ (solid circles) and $22 \mathrm{~nm}$ (open circles); intensity enhancements are 52 and 34, respectively. b, Raw data of energy analyser scans for various wavelengths and fixed total charge (12 $\mathrm{nm}$ tip). Curves represent number of electrons with energies above analyser potential and demonstrate wavelengthdependent cutoff. (A negative bias voltage is needed to repel the negatively charged electrons.) c, Interferometric autocorrelations (electron signal) of pulses at different wavelengths (charge not held fixed for different traces in contrast to $b$ ).

effects (space charge) should be considered [141, 142, 143, 144]. In recent ensemble measurements on dielectric nanospheres with near-infrared light, space charge was found to contribute substantially to particle dynamics [138], which is in contrast to the conditions of our study here. In general, emission from single sharp and moderately voltagebiased metal tips is less susceptible to space charge, in part because of rapidly diverging trajectories. Experimentally, we can verify that space charge effects are insignificant here 
by measuring the kinetic energy distributions at different wavelengths and constant emitted charge (Fig. 4.2b). The strong increase in cutoff energy with wavelength demonstrates that the dynamics are governed not by the total emitted charge, but rather by the properties of the driving field.

To identify the wavelength-scaling of the electron acceleration, conditions at fixed local driving field are desired. Given that the field enhancement generally depends on wavelength, knowledge of the incident intensity alone is insufficient. However, in the adiabatic regime, measurements of total charge and pulse duration can be used to ensure constant local fields. At constant emitted charge and the present field strengths, the pulse durations need only be approximately equal to realize nearly identical local fields; this follows from the fact that the charge in the Fowler-Nordheim model depends linearly on the pulse duration and exponentially on the inverse field. We characterize the pulse durations for different wavelengths in situ by overlapping a pair of identical pulses with variable delay on the tip [53, 57], using the electron signal to produce an interferometric autocorrelation (Fig. 4.2c). The autocorrelations can be used to extract the pulse duration, taking into account the field-dependent tunnelling nonlinearity (Supplementary Information), which leads to the observed sharpening of the oscillatory features at lower fields, visible for example, in the $8-\mu \mathrm{m}$ measurement. The spread in extracted pulse durations (from 60 to $95 \mathrm{fs}$ ) corresponds to a variation of less than $5 \%$ in local field around a value of $21 \mathrm{~V} \mathrm{~nm}^{-1}$ for the curves shown in Fig. 4.2b. This allows us to study the pure wavelength-dependence of the electron dynamics.

From the scaling of the ponderomotive potential $U_{p}$, one may expect the cutoff energies for constant local intensity to depend quadratically on wavelength. We observe a drastic deviation from this behaviour. Instead, we find a strong increase of the kinetic energy for short wavelengths only, followed by a much slower increase, shown for two different local intensities in Fig. 4.3a. The simulations reveal that the observed deviation from regular ponderomotive scaling arises directly from the sub-wavelength decay of the near-field. The data fall into 'corridors' (grey-shaded areas) given by the cutoffs for zero and unity reflection coefficient at the surface. The latter is used in Fig.4.2a, as in ref. [70], but we note that the finite uncertainty in the field enhancement does not allow for a precise determination of the actual reflection coefficients from the data. This might be surprising, because a factor of at least five is expected between the direct and rescattered cutoffs [7]. However, the cutoffs converge at longer wavelengths, a feature unique to strong-field dynamics at nanostructures (see below). 

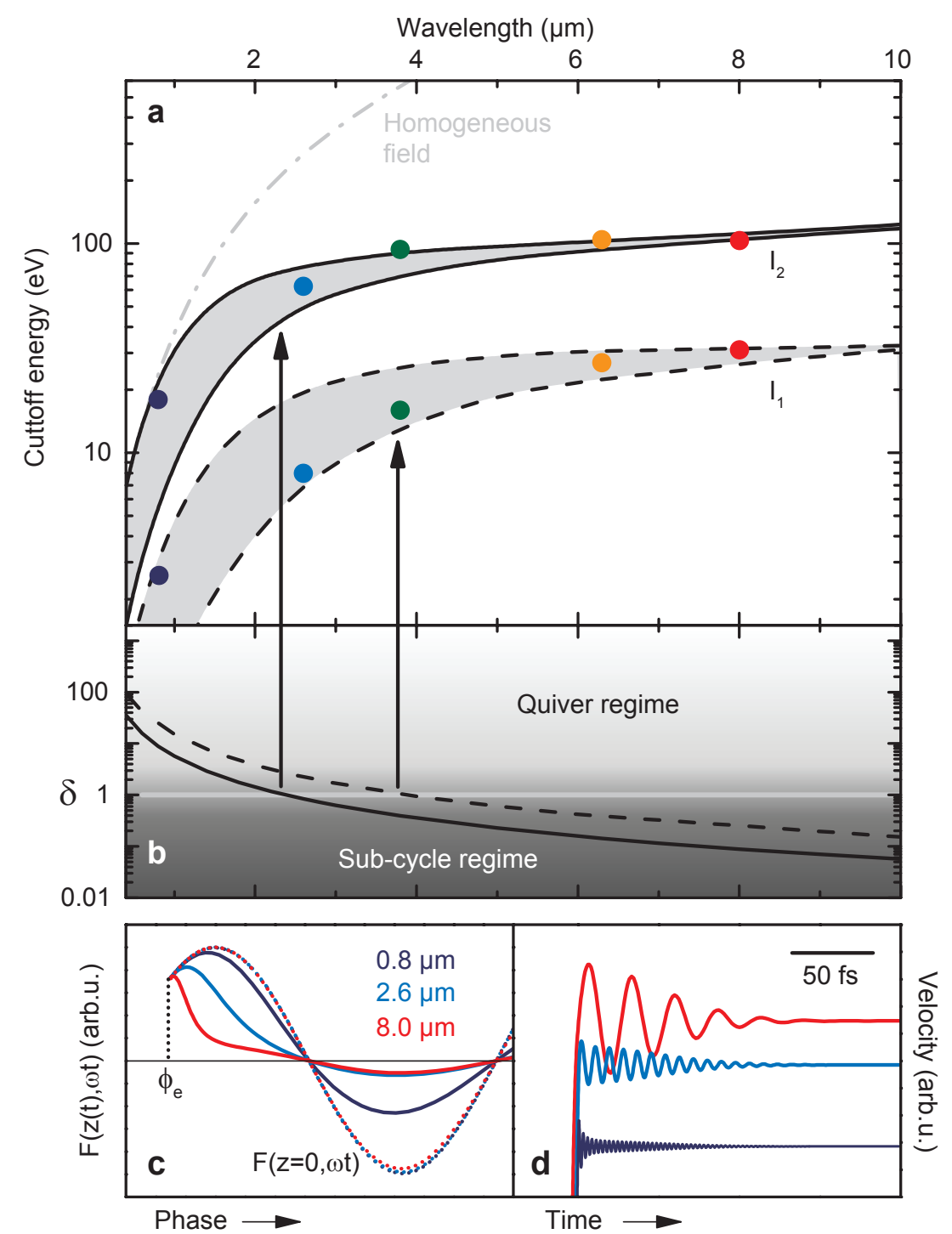

Figure 4.3: Transition from quiver to sub-cycle electron dynamics. a, Experimental cutoff energies versus wavelength for two values of emitted charge (circles). In the adiabatic limit, constant charge and constant intensity are equivalent. The cutoff is defined to include $99 \%$ of the electron population. Simulations (at local intensities $I_{1}=5.4 \mathrm{TW} \mathrm{cm}^{-2}$ and $I_{2}=40 \mathrm{TW} \mathrm{cm}^{-2}$ ) are shown for full rescattering and without any rescattering (upper and lower bounds of the grey 'corridors', respectively), and neglecting the spatial field dependence (dash-dotted grey line). b, Spatial adiabaticity parameter and transition between quiver and sub-cycle regimes corresponding to a. c, Electric field versus phase experienced by propagating electron (emitted at a phase $\phi_{e}$ ) for different wavelengths; $z(t)$ is the distance from the tip. The dotted trace is the field at the surface $(z=0)$. At long wavelengths, the electron leaves the nearfield within a fraction of a half-cycle. d, Corresponding time-dependent electron velocities. For $8 \mu \mathrm{m}$, the electron momentum is attained in much less than one half cycle. 
At the experimental near-field intensities and especially at the long wavelengths used, the quiver amplitude can easily exceed the nanometrescale field decay length. If this happens, the quiver motion is increasingly quenched and the cutoff energy begins to saturate, as predicted by the simulations (Fig. 4.3a, solid and dashed lines). The electron propagation in this localized field suggests the introduction of a new dimensionless adiabaticity parameter that relates the (1/e)-decay length $l_{F}$ of the optical field to the electron quiver amplitude $l_{q}=e F / m \omega^{2}$ as follows:

$$
\delta=\frac{l_{F}}{l_{q}}=\frac{l_{F} m \omega^{2}}{e F}
$$

Whereas the Keldysh parameter describes the degree of adiabaticity of the emission process (that is, in step 1 of the Simpleman model), the ratio $\delta$ characterizes the propagation and acceleration (step 2 in the Simpleman model). For $\delta>>1$, the electron quivers in a nearly homogeneous field over multiple optical cycles (depending on the light pulse duration) in the usual way. For $\delta<<1$, electrons rapidly escape from the tip-enhanced field within a time much shorter than an optical cycle (Fig. $4.3 \mathrm{c}, \mathrm{d}$ ). In fact, the same expression for the $\delta$-parameter follows from the ratio of the escape time at maximum energy $\left(2 U_{p}\right)$ to the optical period, or similarly from the ratio of an electrostatic energy $F l_{F}$ to the ponderomotive energy. In the sub-cycle regime, the electron energy begins to scale sublinearly with intensity and will become linear in the field at even lower driving frequencies.

The transition from quiver to sub-cycle dynamics $(\delta \approx 1)$ occurs within the range of wavelengths used in our experiments, as shown in Fig. 4.3a, b. Near the transition, the cutoff energy becomes wavelength-independent, in agreement with simulation, because the fastest electrons escape the tip-enhanced field well before it completes a half-cycle. Such conditions are only reached in the presence of both high fields and local confinement, as shown by simulations without spatial field dependence.

By means of simulations with fixed (enhanced) surface intensity and variable decay length, we find that several aspects of the electron dynamics change substantially from the quiver to the sub-cycle situation. In the sub-cycle regime, most trajectories display minimal quiver motion, and rescattering occurs over a reduced range of emission phases (Fig. 4.4a, b). Also, the maximum of kinetic energy shifts to later emission phases (Supplementary Information). From the usual maximum-energy values [7] of $2 U_{p}$ and $10 U_{p}$ 

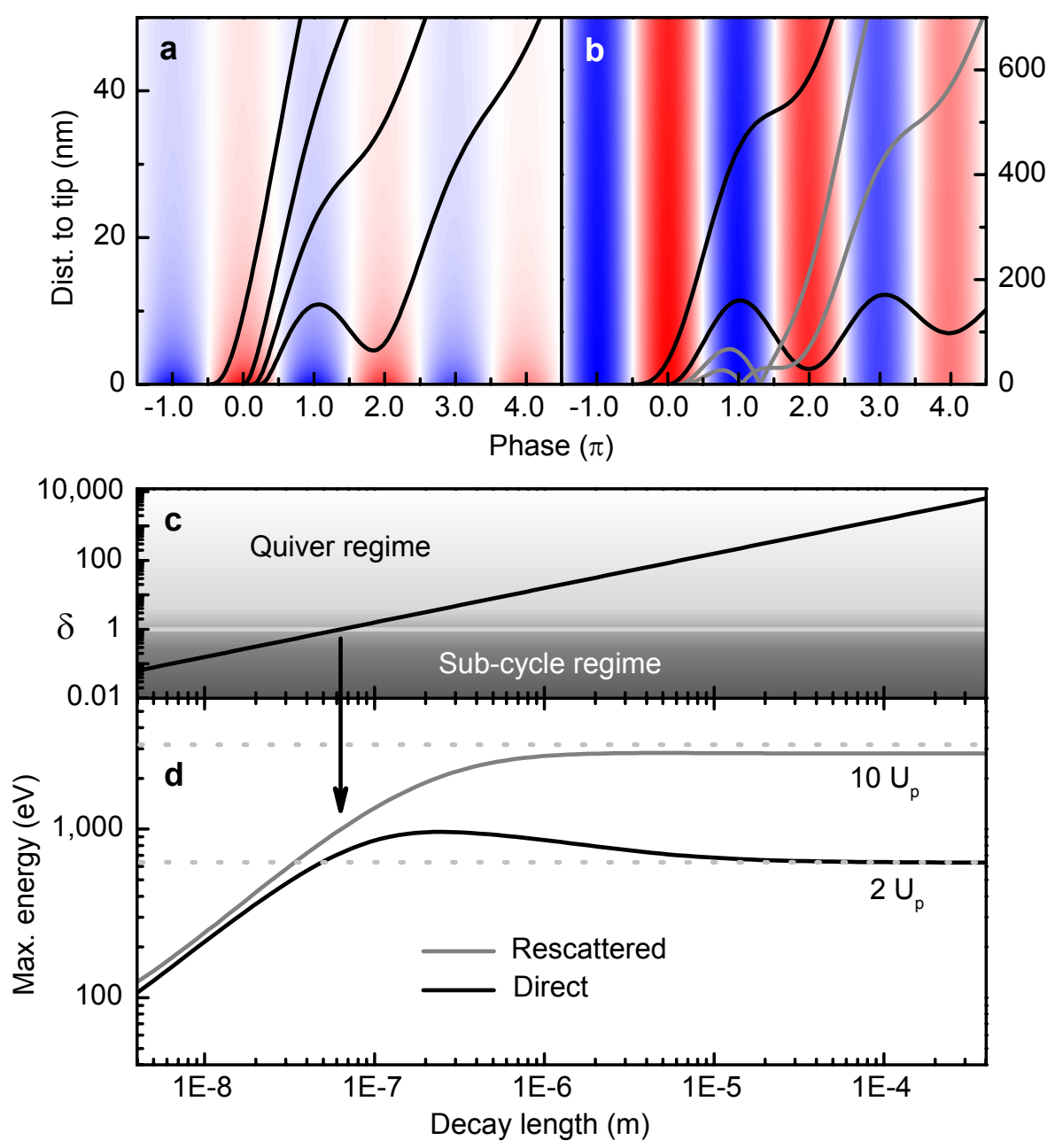

Figure 4.4: Impact of field localization on electron dynamics. a and b, Simulated electron trajectories for four emission phases in localized (a) and homogeneous (b) pulsed fields (wavelength $8 \mu \mathrm{m}$, colour shading indicates field; red and blue indicate positive and negative electric force on electron, respectively). Grey lines are rescattered trajectories. c and d, Spatial adiabaticity parameter (c) and maximum kinetic energies (d) for direct (black line) and rescattered (grey line) trajectories as a function of field decay length for a fixed peak intensity at the surface. Direct and rescattered energies converge at small $\delta$-parameters. $U_{p}$ includes the local intensity enhancement, held constant for all decay lengths in this figure for illustrative purposes.

(defined in terms of local, not incident intensity) at long field decay length, the energies of direct and rescattered electrons become smaller and converge for $\delta \leq 1$ (Fig. 4.4c). This follows from reduced back-acceleration after the first half-cycle, which also causes an energy maximum at intermediate decay lengths for direct trajectories. In the present experiments, the carrier-envelope phase of the pulses is not stabilized. Recent work showed 
the importance of carrier-envelope phase effects, especially for the high-energy cutoff region of the spectra $[70,138]$. Our simulations predict similar features also in the sub-cycle regime studied here, with modifications arising from the fact that the phase of maximum electron energy is shifted for small $\delta$-parameters owing to the field localization (Supplementary Information).

In conclusion, a new regime in strong-field physics is reached by driving the optical near-field in nanostructures with few-cycle mid-infrared pulses. Qualitatively different electron dynamics, for example, regarding the roles of ponderomotive acceleration and backscattering, result from quiver amplitudes that exceed the near-field decay length. Here, electrons escape the driving field within a fraction of the driving period - conditions which would require relativistic energies inside diffraction-limited far-fields. In the extreme limit, the sub-cycle regime encodes the instantaneous light field, rather than its temporal integral, onto the electron energy. This will enable new applications in a strongfield "lab on a tip" with ultrafast streaking and gating at large bandwidth and additional control parameters, such as the static field. The locally accelerated electrons have excellent spatial coherence and may be used as local probes of fields and atomic structures, and in energy-gated schemes, as switchable high-brightness wavepackets for time-resolved surface diffraction.

\section{Methods Summary}

A titanium-sapphire amplifier $(800 \mathrm{~nm}, 1 \mathrm{kHz})$ pumps an optical parametric amplifier. From the signal and idler, tunable mid-infrared pulses $(2.6-8 \mu \mathrm{m})$ are produced by difference frequency generation. After variable attenuation, the pulses are coupled into an ultrahigh-vacuum chamber and focused by a parabolic mirror onto the nanotip. Experiments using attenuated 800-nm pulses are also performed. Raster-scanned shadow images (for example, Fig. 1d) are recorded using the light passing the tip. Autocorrelations of the pulses are obtained with an ultra-broadband interferometer, making use of the nonlinearity of the photoemission process.

Gold nanotips are produced by electrochemical etching, achieving apex radii of 10$20 \mathrm{~nm}$. Each tip is mounted on a three-dimensional micro-translation stage, allowing for raster scans and alignment in the focus. A bias voltage of $-40 \mathrm{~V}$ is applied to the tip to draw the photoelectrons moderately away. Electrons are detected with a microchannel- 
plate phosphor-screen (MCP-PS) assembly. Kinetic energy distributions are measured using a retarding-field energy analyser, correcting for the static offset. A charge-coupled device (CCD) camera records the signal from the MCP-PS as described in ref. [60]. The Supplementary Information contains a sketch of the setup.

Electron spectra are simulated with a two-step model: tunnel emission of photoelectrons and subsequent acceleration in the localized, tip-enhanced laser field. Single-particle trajectories are evaluated as a function of emission phase by solving the equation of motion. The results are weighted by the appropriate tunnelling currents to produce the energy distribution. Spectra are averaged over different carrier-envelope phases. Experimental parameters (wavelength, pulse duration, pulse energy, apex and focus diameters) and intensity enhancements consistent with previous literature are used for different tips. More details are in the Supplementary Information.

\section{Acknowlegements}

We thank R. Bormann, F. Schenk, M. Sivis and S. V. Yalunin for discussions. Financial support by the Deutsche Forschungsgemeinschaft (DFG-ZUK 45/1 and SPP 1391) is gratefully acknowledged.

\section{Author Contributions}

All authors were closely involved in this study and contributed to the ideas, realization of the experiments, data analysis and interpretation, and writing of the paper. 


\section{Supplementary Information, Chapter 4}

\section{Experimental setup}

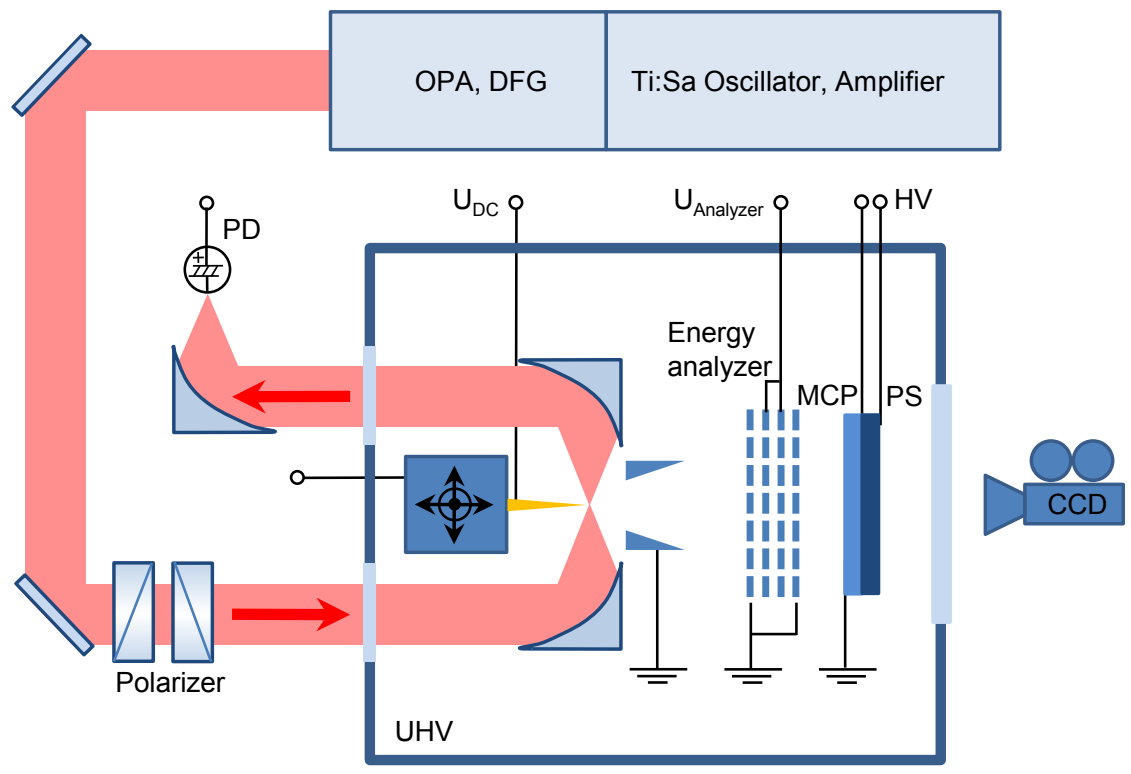

Figure 4.5: Experimental setup used in mid-infrared photoemission experiments. Ti:Sa: titanium-sapphire, OPA: optical parametric amplifier, DFG: difference frequency generation, UHV: ultrahigh-vacuum chamber, UDC: DC-bias voltage, HV: high voltage, PD: photo detector, MCP: microchannel plate, PS: phosphor screen, CCD: charge-coupled-device camera.

\section{Pulse length determination}

The interferometric autocorrelations at different wavelengths are modeled using the FowlerNordheim (FN) equation, taking into account both the peak-to-baseline ratio and the shape (i.e. sharpness) of the individual cycle oscillations, which are both signs of the underlying nonlinearity. Thus, this analysis produces pulse durations corrected for the nonlinearity. The use of the FN equation includes a field-dependent effective nonlinearity, which is highest for the lowest fields and decreases for increasing field strength. Reliable pulse duration determinations are possible using this model for different effective nonlinearities. Figure 4.6 displays experimental and simulated interferometric autocorrelations for different field strengths at $3.8 \mu \mathrm{m}$ wavelength (a), together with magnified views of the central oscillations (b). Extracted pulse durations (bottom to top): $70 \mathrm{fs}, 62 \mathrm{fs}, 67 \mathrm{fs}, 50 \mathrm{fs}$. The first three values lie within uncertainty boundaries, so that we determine an actual pulse 


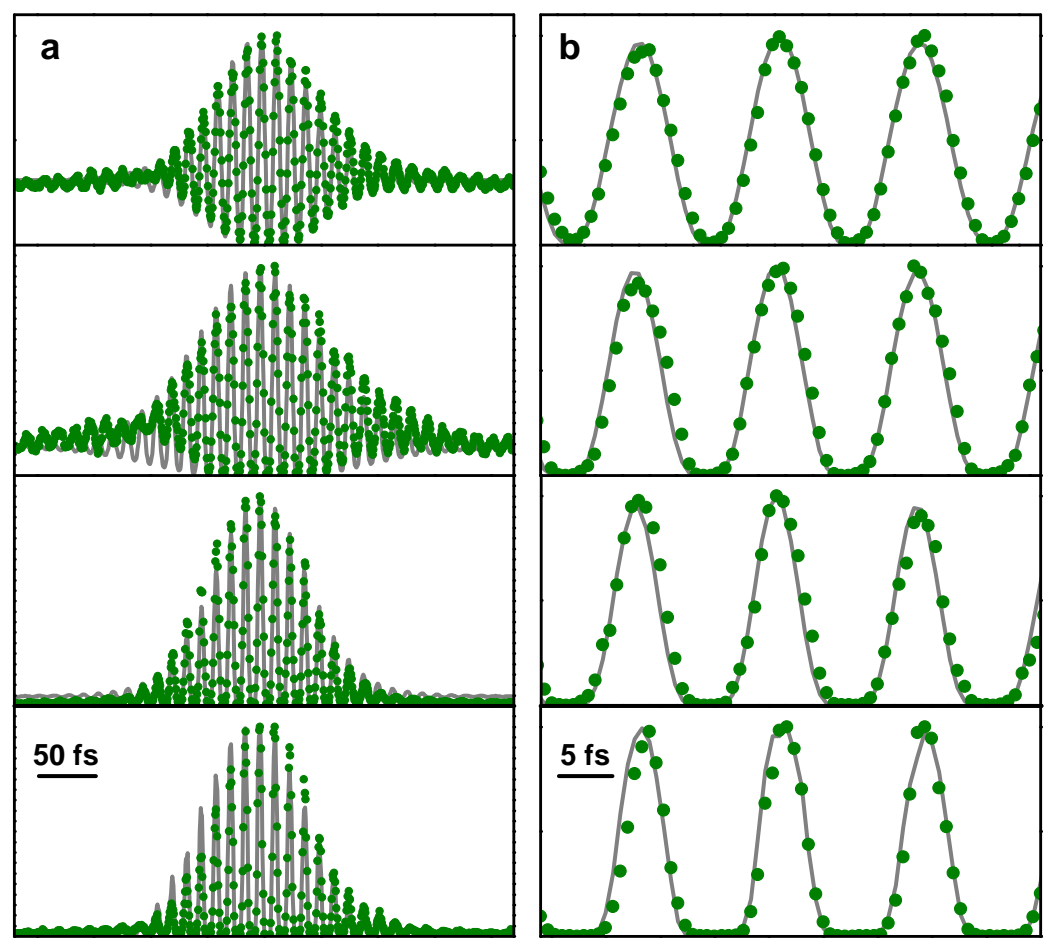

Figure 4.6: Interferometric autocorrelations (IACs) at different field strengths. Experimentally measured IACs (green circles) and corresponding simulated traces (grey) from the FowlerNordheim model. Curves display different nonlinearities, as visible from a varying peak-tobaseline ratio and sharpness of the single oscillations. A $\operatorname{sech}^{2}$-intensity envelope is used.

duration of $65 \pm 5 \mathrm{fs}$. The somewhat shorter value at the lowest nonlinearity arises from the fact that the IAC becomes a field correlation for a linear process, which then contains only information on the spectrum and the Fourier-limited pulse duration, not the actual pulse duration. For this reason, all pulse duration determinations were carried out in the sufficiently nonlinear regime. The same analysis, carried out at wavelengths of $0.8 \mu \mathrm{m}$, $2.6 \mu \mathrm{m}, 6.3 \mu \mathrm{m}$ and $8 \mu \mathrm{m}$, yield pulse durations of $80 \mathrm{fs}, 95 \mathrm{fs}, 60 \mathrm{fs}, 75 \mathrm{fs}$, respectively.

\section{Dependence of peak field on pulse duration}

In the experiments, a constant emitted charge for different wavelengths is set, and the resulting kinetic energy cutoffs are compared. In order to simulate the underlying wavelengthdependence of the kinetic energy spectra and cutoffs, constant driving fields for the acceleration are desired. As described in the manuscript, some variation in the pulse durations does not preclude a fixed field because the emitted charge is a linear function of the pulse duration and (for moderate fields) a nonlinear function of the field. Figure 4.7 displays 


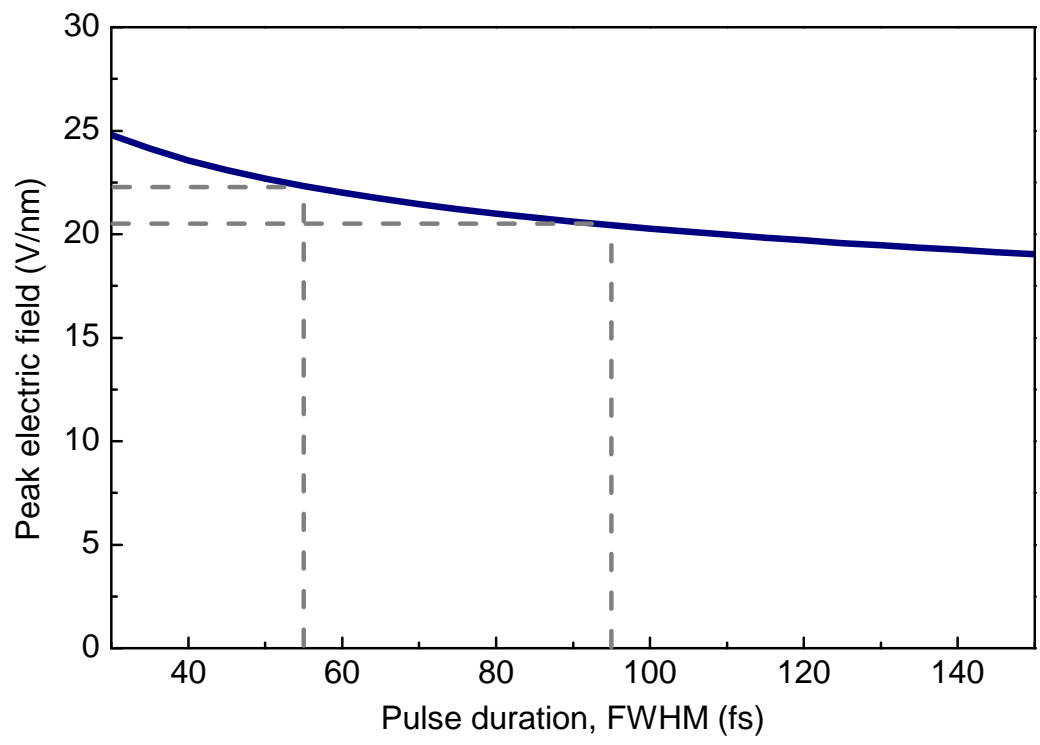

Figure 4.7: Dependence of peak electric field on pulse duration for a fixed emitted charge. The peak field depends weakly on the pulse duration because the emitted charge is a linear function of the pulse duration and a nonlinear function of the field.

the peak electric field as a function of pulse duration for a situation in which the charge is fixed and the local driving field remains in the range of $20-25 \mathrm{~V} / \mathrm{nm}$. It is evident that pulse durations varying even by factors of two correspond to driving fields that differ by only $10 \%$.

\section{Simulation of electron energy spectra}

The electron spectra are simulated with an adapted two-step model consisting of the generation of photoelectrons by tunnel emission and subsequent acceleration in the temporallyand spatially-varying electric field. Since the present experiments involve low frequencies and sufficiently strong fields (low Keldysh parameters), we use a commonly employed form of the Fowler-Nordheim (FN) equation to describe the tunnel current density:

$$
j(t) \propto \Theta\left(-F_{z=0}(t)\right)\left|F_{z=0}(t)\right|^{2} \exp \left(-\frac{4 \sqrt{2 m_{e}} \Phi^{3 / 2}}{3 \hbar e\left|F_{z=0}(t)\right|}\right)
$$

Here, $\Theta$ is the Heaviside function, $\Phi$ is the material work function $(\approx 5.1 \mathrm{eV})$ and $F_{z=0}(t)$ is the time-dependent electric field at the tip surface. Neglecting a field-dependent barrier suppression does not have a major impact on the computed spectra under the present con- 


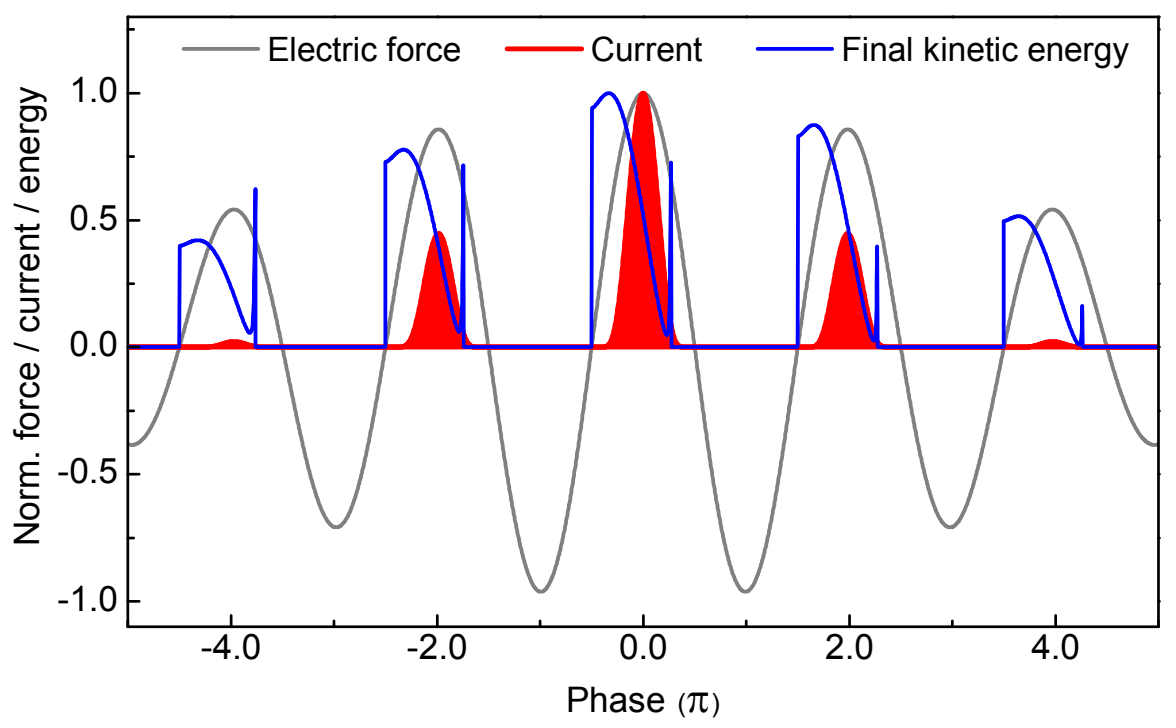

Figure 4.8: Dependence of kinetic energy, current density and electric force on emission phase for a few-cycle pulse. The kinetic energy spectra arise from weighting the final kinetic energies (blue) with the instantaneous tunnel current (red). Wavelength $8 \mu \mathrm{m}$. Only energies from direct electron trajectories are included in the plot. The sharp spikes in the kinetic energy arise from electrons interacting with the subsequent cycle.

ditions. The temporal variation in the electric driving field is modeled by a carrier-wave with a carrier-envelope-offset phase, and an intensity envelope duration corresponding to the average experimental value.

The spatial dependence of the electric field arises from the localized tip-enhanced laser field, the focus of the incident radiation and a variable DC bias voltage applied to the tip. For simplicity, the enhanced near-field is modeled with a dipolar distance dependence, and the far-field is characterized by a Gaussian focus (intensity width derived from focal scans of the nonlinear electron emission and shadow image):

$$
F(z, t)=F_{z=0}(t) \cdot\left[(\alpha-1)\left(\frac{r_{0}}{z+r_{0}}\right)^{3}+\exp \left(\frac{-2 \ln (2) \cdot z^{2}}{\left(2 w_{f o c}\right)^{2}}\right)\right]
$$

Here, $\alpha$ is the field enhancement, $r_{0}$ is the tip radius, $z$ is the distance from the tip and $w_{f o c}$ is the beam waist. For this distance dependence, the (1/e)-decay length used in the $\delta$-parameter corresponds to approximately $0.4 r_{0}$. The additional spatial dependence of the static bias field was taken from finite element simulations.

In this driving field, the single-particle trajectories are evaluated as a function of emis- 
sion time in the pulse by solving the equation of motion with a Runge-Kutta ordinary differential equation solver. For a few-cycle pulse at $8 \mu \mathrm{m}$ wavelength, Figure 4.8 displays the driving force on the liberated electrons (grey), the instantaneous tunnel current density (red) and the emission time-dependent final kinetic energy (blue). These energies are weighted by the corresponding current densities to produce the electron energy distribution. Analogously to focal averaging, the effect of a varying local field enhancement at different emission sites on the tip apex is included to model the experimental situation. The spectra are also averaged over different carrier-envelope phases.

\section{Phase dependence of maximum kinetic energies}

Figures 4.9a,b show the $\delta$-parameter and resulting maximum energies of direct and backscattered electrons in a scenario with fixed $\gamma_{l o c}$ and for varying decay length. As the decay length is being reduced, the energies in the presence and absence of backscattering fall below the usual $10 U_{p}$ and $2 U_{p}$, respectively, and converge at lower values. At very short decay lengths, the maximum energies vary linearly with the field. Figure 4.9c shows the normalized final kinetic energies as a function of the phase at the time of emission. The red (blue) color-coded region corresponds to emission phases which do (do not) result in backscattering. The normalization is carried out in such a way that for every decay length, the maximum energy for the direct and rescattered electrons each correspond to a value of unity. The dashed lines correspond to the phases of maximum energy, whose values are thus given in Fig. 4.9b. It is apparent that for short decay lengths, the emission phases, at which the highest energies are obtained, are shifted. The phases of maximum energy for direct (not backscattered) trajectories shift towards the field maximum, so that the integral over the interaction with the field is maximized. At zero decay length, the phase of maximum energy will approach the field maximum (zero phase). In the present simulations, the energy maximum does not reach this condition, remaining instead at nonzero emission phase because we include the incident far-field radiation, which presents a finite contribution to the energy. The phases of maximum energy for backscattered trajectories are also shifted to larger values, since this allows an earlier time of backscattering with subsequent longer forward acceleration period. Generally, it is observed that the range of values over which backscattering occurs is significantly reduced at very small decay lengths. The stripe-like oscillatory features in the backscattering regions arise from interactions with multiple cycles. 


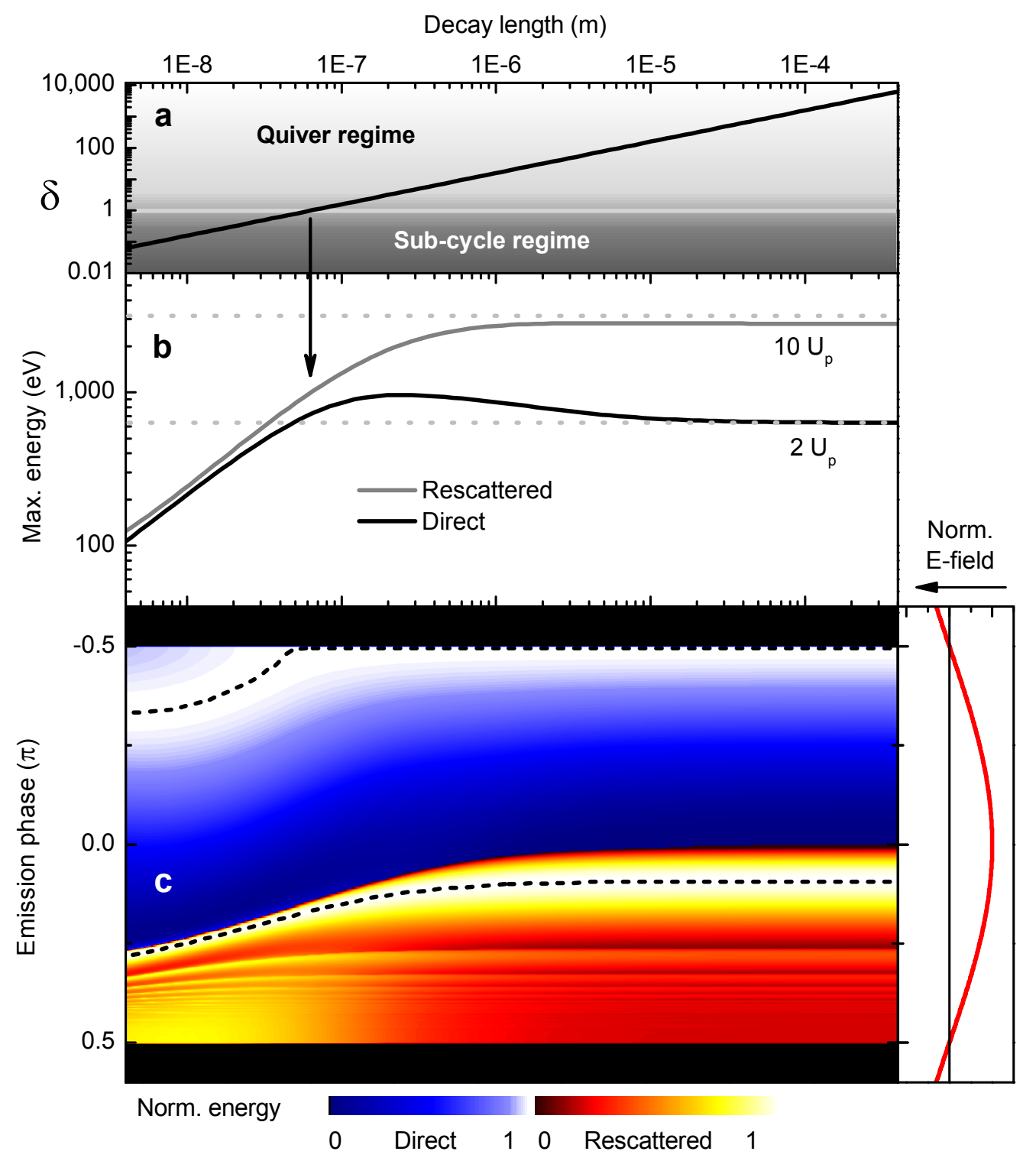

Figure 4.9: Cutoff energy as a function of $\delta$-parameter and phase shift of maximum energies. Spatial adiabaticity parameter (a) and maximum energy (b) as a function of field decay length (for fixed local intensity). c Dependence of final kinetic energy for direct and rescattered electron trajectories as a function of decay length and phase of emission, normalized to their respective energy maximum at each decay length. The dashed lines mark the energy maxima, which are plotted in (b). 
Chapter 4 Field-driven photoemission from nanostructures quenches the quiver motion 


\section{Chapter 5}

\section{Terahertz control of nanotip photoemission}

L. Wimmer ${ }^{1}$, G. Herink ${ }^{1}$, D. R. Solli, S. V. Yalunin, K. E. Echternkamp and C. Ropers Nature Physics 10, pp.432-436, June 2014

DOI: $10.1038 /$ nphys 2974

The active control of matter by strong electromagnetic fields is of growing importance, with applications all across the optical spectrum from the extreme-ultraviolet to the far-infrared. In recent years, phase-stable terahertz fields have shown tremendous potential for observing and manipulating elementary excitations in solids [88, 91, 148]. In the gas phase, on the other hand, driving free charges with terahertz transients provides insight into ultrafast ionization dynamics [129, 130]. Developing such approaches for locally enhanced terahertz fields in nanostructures will create new means to govern electron currents on the nanoscale. Here, we use single-cycle terahertz transients to demonstrate extensive control over nanotip photoelectron emission. The terahertz near-field is shown to either enhance or suppress photocurrents, with the tip acting as an ultrafast rectifying diode [149]. We record phaseresolved sub-cycle dynamics and find spectral compression and expansion arising from electron propagation within the terahertz near-field. These interactions produce rich spectro-temporal features and offer unprecedented control over ultrashort free electron pulses for imaging and diffraction.

Controlling electric charges with external fields is at the heart of modern information technology, with ultimate bandwidths limited by switching speeds in nanoscopic devices. The term light-wave electronics illustrates the anticipated application of opti-

\footnotetext{
${ }^{1}$ These authors contributed equally to this work.
} 


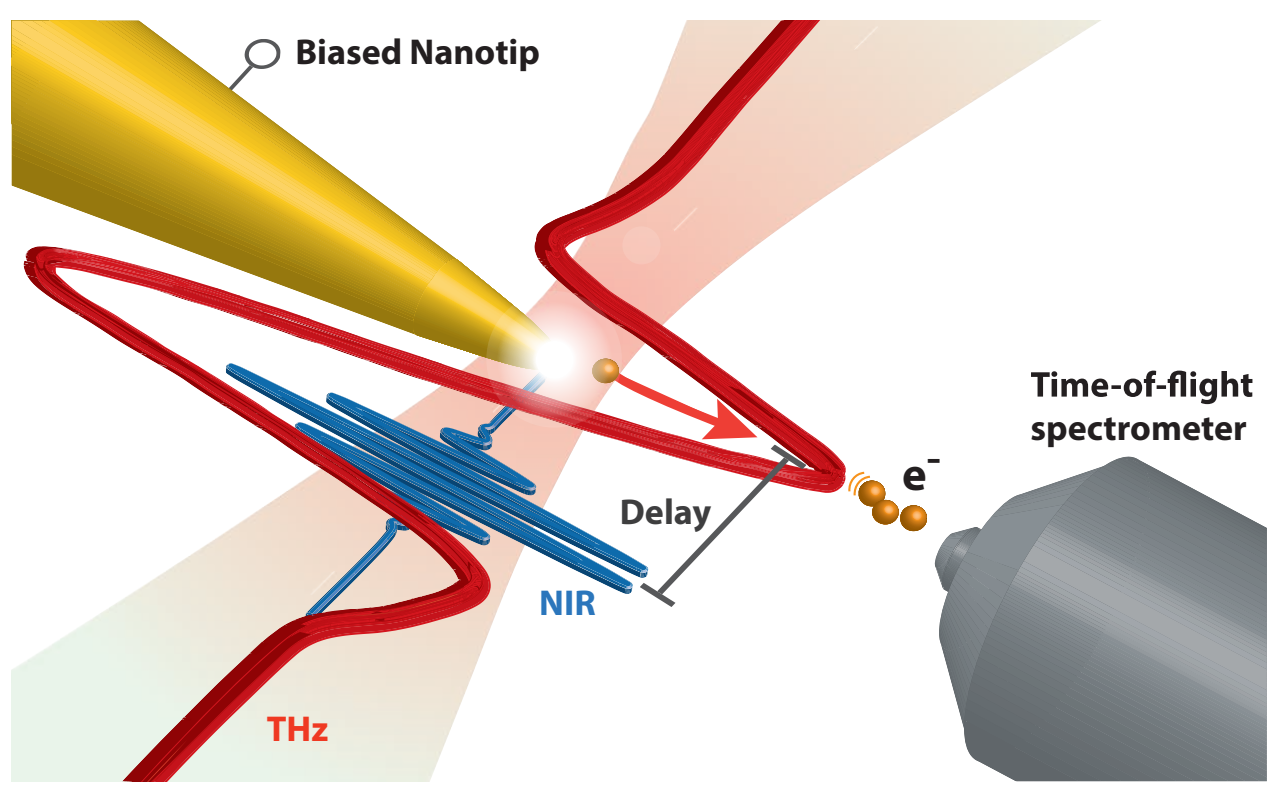

Figure 5.1: Control of nanotip photoemission with terahertz pulses. Ultrashort terahertz (red) and $800 \mathrm{~nm}$ near-infrared (NIR; blue) pulses are focused onto a metallic nanotip. The photoemission current and spectrum are recorded as a function of relative pulse delay.

cally field-driven processes to solids, starting from schemes initially developed for atoms and molecules $[11,150]$. In the terahertz range, strong table-top sources have opened up the field of nonlinear terahertz optics and are enabling comprehensive control over electronic or structural dynamics, for example, in the manipulation of spin waves, the triggering of phase transitions, and the implementation of terahertz-driven scanning tunnelling microscopy [88, 91, 95, 148, 151, 152]. Completely new degrees of freedom are added by employing the localization of optical fields within nanostructures [20, 68, 153, 154]. Specifically, at metallic nanotips, photoelectron emission $[57,62,146]$ with characteristic strongfield features is observed $[60,63,155]$, including carrier-envelope-phase sensitivity $[70,71]$, and sub-cycle electron acceleration at mid-infrared frequencies [20]. A phaseresolved sampling of such processes may be achieved by so-called streaking spectroscopy, a method commonly applied in attosecond science, in which transient fields translate temporal information, for example, instances of ionization, into photoelectron energy or other degrees of freedom $[4,156,157]$.

The application of streaking spectroscopy to metallic nanostructures has been theoretically studied in-depth, aiming primarily at the full temporal characterization of nearinfrared plasmonic fields $[132,133,134,135]$. However, the prospects of transferring 
these concepts to the terahertz domain have not yet been investigated. Here, we show that the enhancement of terahertz fields in nanostructures allows for far-reaching electron trajectory control, spanning from phase-resolved streaking governed by the momentary electric field to propagation-induced spectral reshaping. Experimentally, we demonstrate the terahertz control of electron dynamics using dual-frequency excitation of a gold nanotip: near-infrared pulses ( $800 \mathrm{~nm}$ wavelength, 50-fs pulse duration) induce nonlinear photoelectron emission from the tip apex, which is gated and streaked by locally enhanced single-cycle terahertz fields produced in a light-induced air plasma $[95,96]$. The experiment is sketched in Fig. 5.1 and represents a nanoscopic solid-state version of a terahertz streaking device.

We have recorded photoelectron energy spectra as a function of the delay between the terahertz and near-infrared pulses. This set of measurements comprises a spectrogram, in which both the delay-dependent modulation of the photocurrent and the kinetic energy distribution resolve the action of the terahertz field on the photoelectrons. Figure 5.2 presents such spectrograms for a very sharp tip (10 nm apex radius, Fig. $5.2 \mathrm{~b}$ inset) and two incident terahertz waveforms of opposite polarities (Fig. 5.2a).

For large negative delays, that is, a situation in which the $800 \mathrm{~nm}$ pulse precedes the terahertz pulse, the photoelectron energy is set by the bias voltage (here: $30 \mathrm{~V}$ ), and is unaffected by the terahertz field (Fig. 5.2b,d, delays $<-2$ ps). In the delay range of temporal overlap between the near-infrared and terahertz pulses, the entire kinetic energy distribution is shifted in-phase with a rectifying photocurrent modulation (compare Fig.5.2d,e) of three orders of magnitude, from a more then tenfold enhancement to a $98 \%$ suppression. The delay-dependent photocurrent (Fig. 5.2e) represents a direct measure of the instantaneous terahertz nearfield strengths. This response is caused by a terahertz-modulation of the metal workfunction (Schottky effect) and the opening of lower-order multiphoton channels, as previously observed for static bias voltages [57]. The effect adiabatically follows the local terahertz field because the near-infrared photoemission process is much faster in comparison, ultimately limited by the few-femtosecond dephasing time of the localized tip plasmon [10].

Whereas kinetic energy and photocurrent are strongly linked, some deviations to the incident terahertz field (characterized by electro-optic sampling [158]; Fig. 5.2a) are found, in particular as a small phase shift around the pulse centre and some oscillation at later times. These can be attributed to antenna properties of the conical tip affecting the local field at the apex $[159,160]$, and the present streaking measurements provide direct access 


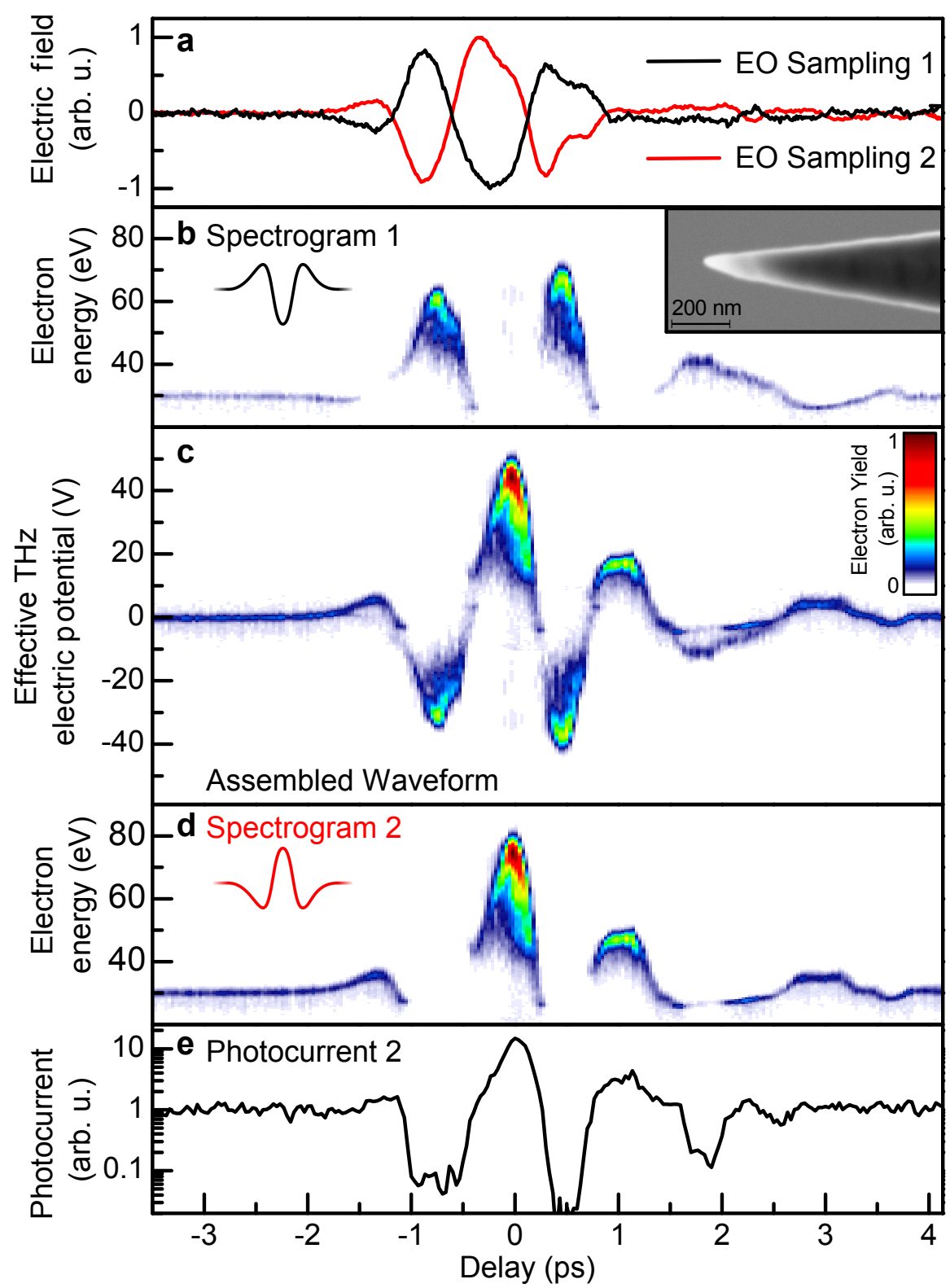

Figure 5.2: Terahertz-streaking spectroscopy of nanotip photoemission. a, Two terahertz transients with inverted field direction detected with electro-optic (EO) sampling at the measurement position of the tip. b, Streaking spectrogram corresponding to EO trace 1. Inset: scanning electron micrograph of the nanotip used (radius: $10 \mathrm{~nm}$ ). c, Terahertz electric waveform derived from the measurements shown in b,d (see text). d, Streaking spectrogram of the waveform corresponding to EO trace 2. e, Photocurrent modulation from spectrogram 2 (shown in d). 
to this local field (Supplementary Information).

The fact that the energy spectrogram (Fig. 5.2b,d) follows the local terahertz field inphase is in stark contrast to common implementations of streaking in diffraction-limited laser foci, including attosecond streaking [4] and terahertz schemes to characterize Xray and extreme-ultraviolet pulses $[129,161]$. Under such conditions, the streaking field is spatially homogeneous. For example, photoelectrons from an atomic gas experience the entire evolution of the streaking field $F(t)$ after the time of emission $t_{e}$, resulting in the electron momentum $P_{\text {streak }}$ governed by the temporal integral over the field, that is, the vector potential $A\left(t_{e}\right): P_{\text {streak }}=e A\left(t_{e}\right)=e \int_{t_{e}}^{\infty} F\left(t^{\prime}\right) d t^{\prime}$ (ref. [4]). In contrast, for the very sharp tip used in Fig. 5.2, the nanolocalized streaking geometry allows electrons to escape the enhanced near-field within a small fraction of the terahertz oscillation period, yielding energies $E_{\text {streak }}$ given by the instantaneous electrical potential [128]: $E_{\text {streak }} \approx e U\left(t_{e}\right)=\int F\left(r, t_{e}\right) d r$. For a surface electric field decaying over a short length $l_{F}$ and in the quasi-static limit, the energy gained is directly proportional to $l_{F} e F\left(r=0, t_{e}\right)$. With decay lengths of only a few tens of nanometres and maximum energies of tens of electronvolts, local terahertz electric fields $F \approx E_{\text {streak }} / e l_{F}$ of multiple $\mathrm{MV} \mathrm{cm}^{-1}$ are derived. As a result of the rectifying characteristic of this field emission geometry, the spectrograms exhibit gaps at times with strong electric force pointing towards the tip. To characterize the full temporal evolution of the terahertz near-field, we record two spectrograms using opposite polarities in the terahertz transient (Fig. 5.2b,d). Taking into account the respective polarities (Supplementary Information), these two complementary measurements can be superimposed to obtain - in analogy to attosecond streaking representations [4] - the smooth and continuous local terahertz electric waveform (Fig. 5.2d).

The extreme sub-cycle reduction of the interaction time with a localized driving field represents a limiting case of the recently observed quenching of the quiver motion at nanostructures [20], and is phase-resolved here for the first time. The spatial adiabaticity parameter $\delta=l_{F} m \omega^{2} / e F$ relates the near-field decay length to an electron's quiver amplitude in an oscillating field and describes how closely the final energy follows the instantaneous electric field ( $\omega$ : excitation frequency, $m$ : electron mass) [20]. Generally, the present conditions imply quasi-static acceleration, with $\delta$-values far below unity (for example, $\delta=0.01$ for $F=2 \mathrm{MV} / \mathrm{cm}^{-1}, l_{F}=10 \mathrm{~nm}$ and at $1 \mathrm{THz}$ frequency). However, close to the zero-crossings of the transient, a finite interaction time with the terahertz near-field becomes noticeable. At these delay times, where the photocurrent reappears after suppression ( $\tau=-0.5 \mathrm{ps}$ and $+0.75 \mathrm{ps}$ in Fig. $5.2 \mathrm{~d}$ ), the onset of the spectrogram is 

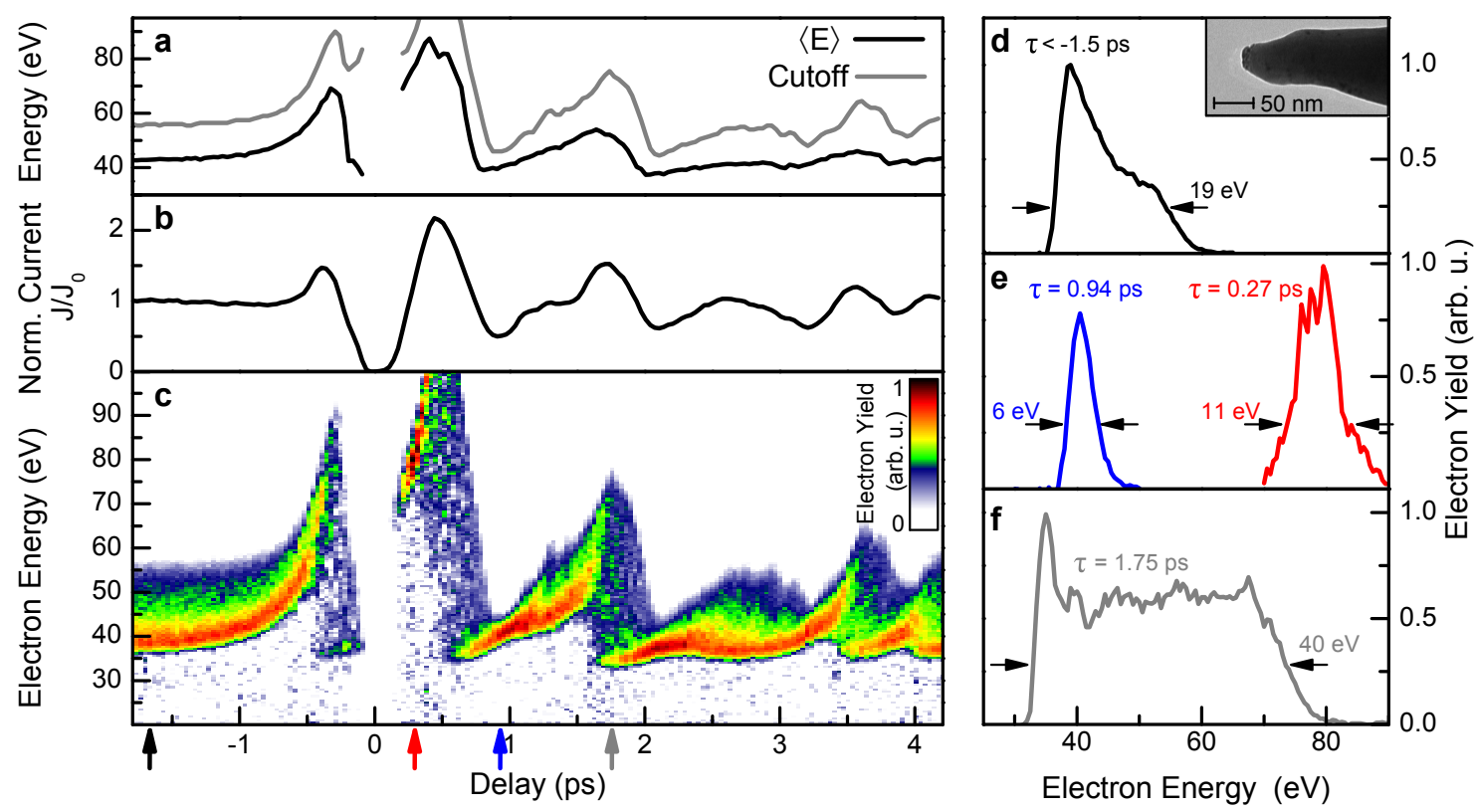

Figure 5.4: Spectral reshaping observed in streaking spectroscopy. a, Expectation value of the energy distributions (black) and cutoff energy (grey) of the delay-dependent spectra. The highenergy cutoff is defined to include $95 \%$ of the electron distribution. b, Normalized photoemission current. c, Spectrogram. d, Energy spectrum unaffected by the terahertz pulse (large negative delay). Inset: transmission electron micrograph of the nanotip used. e,f, Individual spectra at selected delays showing terahertz-induced compression (blue), shifting (red) and expansion (grey). Coloured arrows in $\mathrm{c}$ indicate delay times of respective spectra (static bias: $-40 \mathrm{~V})$.

slightly shifted above the bias voltage from electrons accumulating additional energy during propagation. While being almost negligible for the sharp tip used in the measurements of Fig. 5.2, such propagation effects become more pronounced with increasing tip radius, and in particular, will strongly depend on the initial velocity of photoemitted electrons traversing the near-field.

To investigate such more complex electron dynamics, we have carried out measurements using a tip with larger field decay length and a broader 'primary' photoemission spectrum induced by higher local near-infrared intensity. Figure 5.4 summarizes the results of this experiment, showing the delay-dependent modulation of the expectation value $\langle E\rangle$ of the electron energy distributions and the high-energy cutoff (Fig. 5.4a), the photocurrent modulation (Fig. 5.4b) and the spectrogram (Fig. 5.4c). In the broader ensemble of initial energies generated by the near-infrared pulse alone (Fig. 5.4d), electrons probe the spatially and temporally varying field over different time intervals. Specifically, faster 
electrons rapidly sample the momentary terahertz field as they leave the apex region, while slower electrons experience integration over a time-varying transient. This has profound consequences in spectral redistributions, as evidenced by shape modulations in the spectrogram and the variation in the difference between $\langle E\rangle$ and the high-energy cutoff. As a general result, we find substantial terahertz-induced spectral broadening and compression (Fig. 5.4e,f) as inherent features of propagation within a localized oscillating field. A temporally growing force causes spectral compression because initially slower electrons, which interact over a longer period with the rising terahertz field, experience more overall acceleration than initially faster electrons (compare sketch in Fig. 5.4f). Compression is apparent for various delays, for example, at $-0.1 \mathrm{ps},+1.0 \mathrm{ps},+2.0 \mathrm{ps}$. Spectral expansion, on the other hand, is a feature of a decreasing electric force: fast electrons leave the high field quasi-instantaneously, while slower electrons see a reduced potential, and are even decelerated as the force changes sign, see, for example, delays around $-0.3 \mathrm{ps}$, $+0.6 \mathrm{ps},+1.7 \mathrm{ps}$. We note that these findings represent a nano-optical equivalent of radiofrequency spectral and temporal compression of ultrafast electron pulses, yet at orders of magnitude smaller temporal and spatial scales [162].

Numerical modelling of the streaking spectrograms allows for a comprehensive description of the spatio-temporal acceleration process. Figure 5.5 presents results of a particle propagation simulation for a terahertz transient resembling that in Fig. 5.4. In the model, electron trajectories exposed to the locally enhanced terahertz field are computed for different emission times relative to the streaking transient, and for a distribution of initial velocities (Supplementary Information). Considering the different timescales involved, we can separate the effects on the electron spectra induced by the near-infrared photoemission field and by the terahertz streaking field: the near-infrared field causes the broadening of the initial kinetic energy spectrum by strong-field emission and acceleration $[20,60,63]$, as evident in the absence of the terahertz field at negative delays. In contrast, the terahertz field provides the shift and reshaping of the final spectra after emission. This approach is justified by the more than two orders of magnitude differing frequencies at which emission and streaking occur.

Figure 5.5a shows selected spectra simulated for various delay times, illustrating the strong spectral shaping induced by propagation in the spatially confined terahertz field (decay length $40 \mathrm{~nm}$ ), see, for example, the spectral narrowing (blue, red) starting from the initially broader spectrum (black). The transfer function from initial to final energies for two selected emission times is plotted in Fig. 5.5b, while Fig. 5.5c shows the temporal 


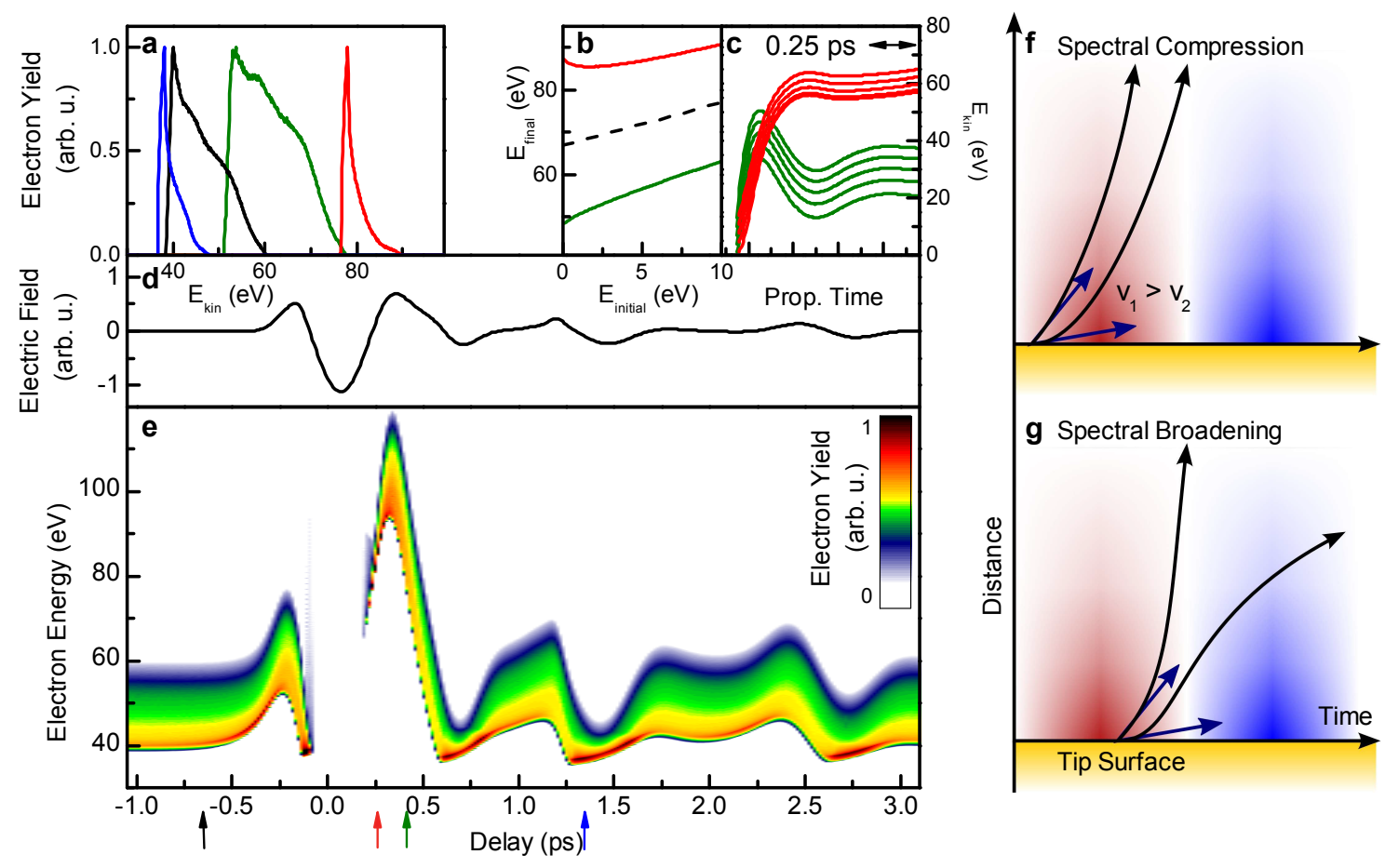

Figure 5.5: Simulation of terahertz streaking at a nanotip. a, Energy spectra at different delay times. b, Final electron energy as a function of initial energy for two selected emission times corresponding to the spectra in a. Spectral compression (red) and broadening (green) are apparent (dashed line: unity slope for comparison). c, Temporal evolution of a set of five initial energies for these two emission times. Note that electrons have not yet acquired the full static potential within the plotted interval. d, Terahertz waveform as employed in the simulation. e, Simulated spectrogram. Colour-coded arrows indicate selected delays used in panels ac. f,g, Illustrations of spectral compression (f) and broadening (g) for two different initial velocities $\left(v_{1}, v_{2}\right.$; blue arrows) induced by temporally rising and falling near-fields, respectively (red/blue: positive/negative force).

evolution of a set of energies at two delay times resulting in spectral broadening (green) or compression (red). The full delay-dependent spectrogram (Fig. 5.5e) reproduces all main features of the measurements, including the terahertz-field-induced energy shift, current enhancement and suppression (Fig. 5.5d), as well as complex spectral reshaping. Even subtle characteristics, such as the narrow low-energy lobe at small negative delays, are successfully reproduced. The model computations allow us to quantitatively determine the magnitude of the local terahertz field. We obtain a peak field strength of $9 \mathrm{MVcm}^{-1}$, which amounts to a local field enhancement at the tip of about 90. At frequencies of only a few terahertz, such high fields are very hard to achieve in the absence of local field enhancements [95, 96, 163], and are close to the threshold for terahertz-induced 
tunnelling [164] (Supplementary Information).

In conclusion, we demonstrate high-contrast switching and control of photocurrents and photoelectron spectra by tip-enhanced single-cycle terahertz pulses. In essence, the results represent the implementation of an ultrafast rectifying diode for the terahertz field, which is triggered by the near-infrared pulse. The spectral broadening and compression shown here amounts to a flexible tuning of the electron phase space density. The concept can be extended to other frequency ranges, provided that the involved spatial, temporal and energetic scales are suitably coupled along the particle trajectories. The approach has significant potential for the spectral and temporal optimization of electron pulses used in ultrafast electron diffraction and transmission electron microscopy, and may lead to a tailored focusing of electron pulses at controlled distances from the nanocathode.

\section{Acknowledgements}

We thank K. Reimann, M.Wörner and T. Kampfrath for helpful discussions and B. Schröder, S. Schnell and A. Feist for technical support. We gratefully acknowledge financial support by the Deutsche Forschungsgemeinschaft (DFG-SPP 1391 'Ultrafast Nanooptics' and $\mathrm{ZuK} 45 / 1$ ).

\section{Author contributions}

L.W., G.H. and C.R. conceived and designed the experiment, which was set up by L.W., G.H., K.E.E. and D.R.S.. Experiments were carried out by L.W. and G.H., and the data were analysed by L.W., G.H. and C.R. The simulations were implemented by G.H., and an analytical model to determine simulation parameters was implemented by S.V.Y. All authors discussed the results and contributed to the interpretation, and the manuscript was written by L.W., G.H. and C.R.

\section{Supplementary Information, Chapter 5}

\section{Experiment}

Near-infrared pulses of $800 \mathrm{~nm}$ wavelength, $50 \mathrm{fs}$ duration and at $1 \mathrm{kHz}$ repetition rate are focused onto the apex of single electrochemically-etched gold nanotips with an incident 
peak intensity up to $250 \mathrm{GW} / \mathrm{cm}^{2}(10 \mu \mathrm{m}$ beam waist). The phase-stable THz-transient is generated in a light-induced air plasma [95, 96], pumped by $1.2 \mathrm{~mJ}$ NIR-pulses, and combined with second harmonic generation from a $100 \mu \mathrm{m}$ thick BBO crystal. The polarity of the incident waveform (Fig. 5.2a, red) can be reversed by rotating the BBO crystal by $180^{\circ}$ around the beam direction (Fig. 5.2a, black). Both NIR and THz pulses are coupled into an ultra-high-vacuum chamber and collinearly focused on the tip with variable time delay. We note that the incident $\mathrm{THz}$ spectrum depends on the precise measurement position relative to the central beam axis, with higher frequencies being more focused. Therefore, we have characterized the $\mathrm{THz}$ transient by electro-optic sampling at the exact position of the nanotip in the measurements. Electro-optic sampling was carried out with a ZnTe crystal attached to the sample translation stage (peak field around $100 \mathrm{kV} / \mathrm{cm}$ ). The $\mathrm{THz}$ beam waist is about $900 \mu \mathrm{m}$ at a Rayleigh range of $2 \mathrm{~mm}$. Photoelectrons are detected by a time-of-flight electron spectrometer (Fig. 5.2) or by a retarding-field analyzer with a microchannel-plate anode-assembly (Fig. 5.4) in combination with a fast oscilloscope.

\section{Estimation of THz-induced tunnel emission}

Under the present experimental conditions, we do not observe THz-induced field emission and derive local $\mathrm{THz}$ field strengths around 1-2 V/nm. In static field-emission experiments with such tips, we detect field-emitted currents as low as $1 \mathrm{fA}$, corresponding to local fields of about $2 \mathrm{~V} / \mathrm{nm}$.

However, because of the extremely low duty-cycle in the application of THz-fields (about $100 \mathrm{fs}$ within $1 \mathrm{~ms}$ ), peak currents above $16 \mathrm{nA}$ would be required for a significant detection limit of 1 electron per 100 laser pulses. This corresponds to a peak THz field of around $4 \mathrm{~V} / \mathrm{nm}$ following a Fowler-Nordheim tunneling characteristic. Thus, a moderate increase of the $\mathrm{THz}$ field due to tighter focusing, higher laser pump pulse energy or a nanostructure providing higher field-enhancement may enable the observation of $\mathrm{THz}$ tunneling in future experiments.

\section{Assembly of a time-continuous local waveform}

In the streaking spectrograms, the local $\mathrm{THz}$ surface electric potential is imprinted onto the electron kinetic energy. The electric field along the tip axis decays with a power law of the order 1-2 in the distance and at long wavelengths. The spatio-temporal electron dynamics are mostly governed by the initially fast spatial decay, and the corresponding 
waveform can be directly read from the spectrograms. Only part of the entire potential is converted to kinetic energy during the $\mathrm{THz}$ period. Therefore, the spectrograms represent an effective THz potential $S_{1}(E, \tau)$ (Fig. 5.2d), which contains that part of the total local $\mathrm{THz}$ potential which is effectively converted to kinetic energy during the pulse. Here, $E$ is the measured kinetic energy coordinate and $\tau$ is the delay. The total local THz potential can be obtained using numerical simulations, and we find that it is several times larger than the effective potential, depending on tip geometry. Due to the rectifying character of the field-emission and under the present conditions of low static bias potential, information on times of positive THz field polarity is lost. A second spectrogram $S_{2}(E, \tau)$ (Fig. 5.2b) of a corresponding waveform with reversed polarity (two EOS traces shown in Fig. 5.2a) contains the complementary data, providing the full information on the time-continuous local waveform. As the static bias potential $U_{\text {bias }}$ mainly results in a linear shift along the energy axis, we apply a simple axis transformation to combine spectrograms $S_{1}$ and $S_{2}$ into a single waveform:

$$
S_{t o t}=S_{1}\left(E-U_{\text {bias }}\right)+S_{2}\left(-E+U_{\text {bias }}\right)
$$

Subtracting the static bias potential, this waveform then contains continuous information on the THz-induced electric potential. Adding both spectrograms on a common energy scale yields the complete waveform $S_{t o t}$ - we note that the energy range between -30 $\mathrm{V}$ to $30 \mathrm{~V}$ is overlapping. As a result, the inversion of the energy scale for one of the spectrograms leads to a proper continuity in the waveform and generally good overlap at delays where both spectrograms contain information.

\section{Spectrogram simulation}

The streaking spectrogram in Fig. 5.5 is simulated in a two-step model. First, electron trajectories are calculated for varying initial velocity and emission time in the $\mathrm{THz}$ nearfield, yielding final kinetic energies as a function of initial energy. For simplicity, we consider a one-dimensional propagation characterized by a single field decay length. A typical $\mathrm{THz}$ transient close to the EO-sampling trace corresponding to the measurement in Fig. 5.4 was employed as a local THz near-field. The spatial decay of the THz field and the static field along the tip axis is modeled using $r^{-1}$-dependence, where $\mathrm{r}$ is the distance from the tip, in agreement with numerical solutions of Maxwell's equations for the tip 
geometry:

$$
F(r) \propto \frac{F_{r=0} l_{F}}{l_{F}+r},
$$

with the field decay length $l_{F}=40 \mathrm{~nm}$.

The bias-voltage induced static field is accounted for in the same way, using a maximum field $E_{\text {stat }}(r=0)=U_{\text {bias }} /\left(k_{\text {geo }} R\right)$ with the tip radius $R=40 \mathrm{~nm}$ and a geometrical factor $k_{\text {geo }}=2.5$. A maximum $\mathrm{THz}$ field strength of $9 \mathrm{MV} / \mathrm{cm}$ has to be used to account for the experimentally observed spectral features in Fig. 5.4, e.g., the maximum kinetic energy. The primary NIR-induced kinetic energy distributions are modeled corresponding to those measured in the absence of the $\mathrm{THz}$ field. The final energy distributions are then obtained by applying the computed energy transfer function $E_{\text {final }}\left(E_{\text {init }}\right)$ to the initial distribution.

\section{Antenna Model}

The assembled waveform in Fig. 5.2c follows the corresponding EO-sampling trace of the incident THz-transient in Fig. 5.2a. For an in-depth inspection of the local THz-response of the nanotip, we compare the incident waveform with the energy expectation value of the assembled spectrogram (Fig. 5.6b), representing the local THz-field transient. One observes a small phase-shift of the maximum field, a subtle redistribution of the relative heights of the two lobes and the appearance of a post-oscillation. All these combined features are consistent with previous results on related near-field characterizations in scanning near-field optical microscopy and a generic antenna-model [159, 160], assigning an equivalent electrical circuit to the conical metal tip. Here, the electric charge $q$ that determines the local electric potential at the tip apex is driven by the incident THz-waveform, constituting a transient potential $u(t)$ :

$$
u(t)=L \ddot{q}+R \dot{q}+\frac{q}{C}, \quad f_{0}=\frac{1}{2} \sqrt{\frac{1}{L C}}
$$

Thus, the electrical response is lumped into three reactive parameters which we could assign as capacitance $C=0.35 \mathrm{fF}$, inductance $L=0.1 \mathrm{nH}$ and resistance $R=300 \mathrm{Ohm}$. The corresponding THz-driven RLC circuit features a slightly detuned resonance frequency $f_{0}=0.85 \mathrm{THz}$ with respect to the incident $\mathrm{THz}$ pulse spectrum. The electrical response to the incident waveform (Fig. 5.6a) is shown in Fig. 5.6c, yielding good overall agreement. 

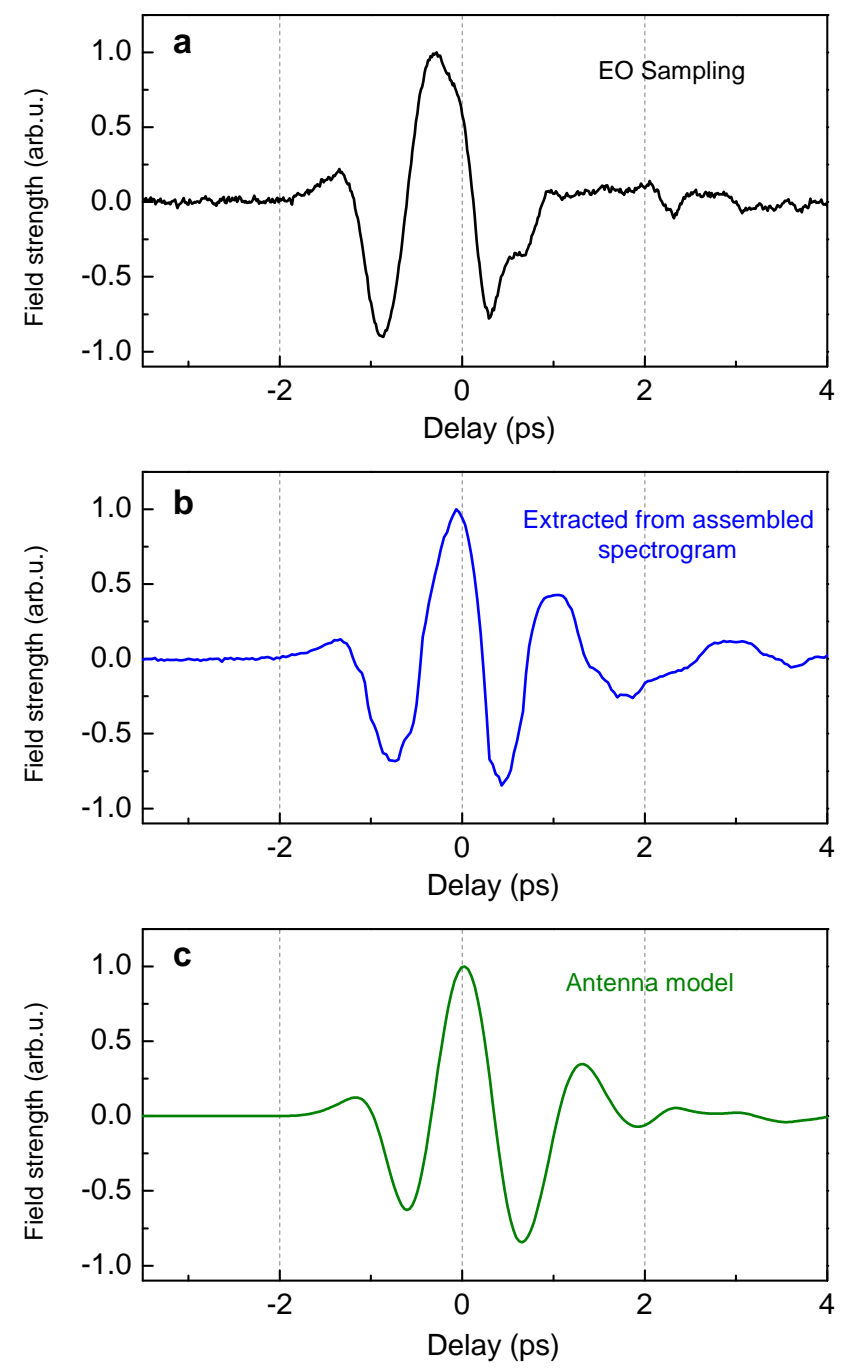

Figure 5.6: The incident $\mathrm{THz}$ waveform is characterized at the tip position (a). The waveform of the local THz field at the apex of the tip is assembled from two spectrograms of opposing polarity by evaluating the energy expectation value (b). The slight phase-shift of the maximum field, subtle redistributions of the relative height of the extremes and the appearance of a postoscillation in the local field compared to the incident waveform can all be reproduced in a first approximation by a generic antenna model (c). 


\title{
Chapter 6
}

\section{Field emission at Terahertz frequencies: AC-tunneling and ultrafast carrier dynamics in nanotips}

\author{
G. Herink, L. Wimmer and C. Ropers \\ New Journal of Physics 16, 123005, December 2014 \\ DOI: $10.1088 / 1367-2630 / 16 / 12 / 123005$
}

We demonstrate ultrafast Terahertz (THz) field emission from a tungsten nanotip enabled by local field enhancement. Characteristic electron spectra which result from acceleration in the $\mathbf{T H z}$ near-field are found. Employing a dual frequency pump-probe scheme, we temporally resolve different nonlinear photoemission processes induced by coupling near-infrared (NIR) and THz pulses. In the order of increasing THz field strength, we observe THz streaking, THz-induced barrier reduction (Schottky effect) and THz field emission. At intense NIR excitation, the THz field emission is used as an ultrashort, local probe of hot electron dynamics in the apex. A first application of this scheme indicates a decreased carrier cooling rate in the confined tip geometry. Summarizing the results at various excitation conditions, we present a comprehensive picture of the distinct regimes in ultrafast photoemission in the near- and far-infrared.

Terahertz radiation with a photon energy of few millielectron-volts (meV) is commonly regarded as being non-ionizing, and finds widespread applications in imaging, sensing and spectroscopy $[88,147,165]$. Until recently, powerful table-top THz sources have been lacking - a situation frequently described as the "Terahertz gap". Yet, modern generation schemes based on femtosecond lasers now provide free-propagating electri- 
cal transients with intrinsic carrier-envelope-phase (CEP) stability and peak fields up to $0.1 \mathrm{~V} / \mathrm{nm}[89,166]$. Avoiding structural damage encountered at visible frequencies, modern high-field THz sources offer ideal opportunities to study nonlinear, field-driven processes [91, 92], including the application of rectified currents in a scanning tunneling microscope [164], control of ultrashort electron pulses [21, 167, 168] or interband tunneling in semiconductors $[169,170]$. The process of field emission typically requires higher field strengths of several V/nm [44, 45]. Thus, AC-tunneling into vacuum, which was observed in microwave resonators in the early 1960s [51, 52], and more recently with ultrafast lasers in the infrared range [20, 171], has not yet been shown in the $\mathrm{THz}$ spectral region. In this work, we demonstrate $\mathrm{THz}$ field emission enabled by the high field enhancement at tungsten nanotips. Electron kinetic energy distributions are used to quantitatively characterize the time-dependent local THz field. Moreover, the ultrashort temporal window of $\mathrm{THz}$ field emission allows us to resolve the hot carrier relaxation in the tip after NIR excitation.

In the experiment, sketched in Fig. 6.1a, we study THz-induced field emission from a sharp tungsten tip (tip diameter $10 \mathrm{~nm}$, biased at a potential $\mathrm{U}_{\text {bias }}$ ) with a time-of-flight (TOF) electron spectrometer ( $3 \mathrm{~mm}$ distance between tip apex and entrance aperture) and a microchannel plate detector. The single-cycle $\mathrm{THz}$ transient is generated in a laserinduced air-plasma by mixing the fundamental wave at $800 \mathrm{~nm}$ wavelength (Ti:Sapphire amplifier system with 50 -fs pulses, $1.7 \mathrm{~mJ}$ pulse energy at $1 \mathrm{kHz}$ repetition rate) with a weak second harmonic wave, generated in a $100 \mu \mathrm{m}$ thick BBO crystal $[95,96]$. The $\mathrm{THz}$ field strength is controlled by rotation of the BBO crystal (Fig. 6.1g), and the pulses are coupled into an high vacuum chamber $\left(10^{-8}\right.$ mbar range) through a $500 \mu \mathrm{m}$ thick, Brewster-angled silicon window. Focussing is achieved with an off-axis parabolic gold mirror of $25 \mathrm{~mm}$ focal length. The THz transient is characterized via pyroelectric detection, electro-optic sampling and the recently developed nanotip streaking [21] inside the vacuum chamber. The high field enhancement for polarization parallel to the tip axis effectively acts as a polarizing element.

We have found that the $\mathrm{THz}$ pulses induce highly nonlinear electron emission from the nanotip, and we first investigate the field emission arising from the $\mathrm{THz}$ transient alone. Electron emission is detected while scanning the nanotip through the $\mathrm{THz}$ focus $(25 \mathrm{~nJ}$ pulse energy), employing the electron yield for nanotip positioning. Figures 6.1c,e show such an emission map and a linescan in the focal plane, respectively. After tunneling 

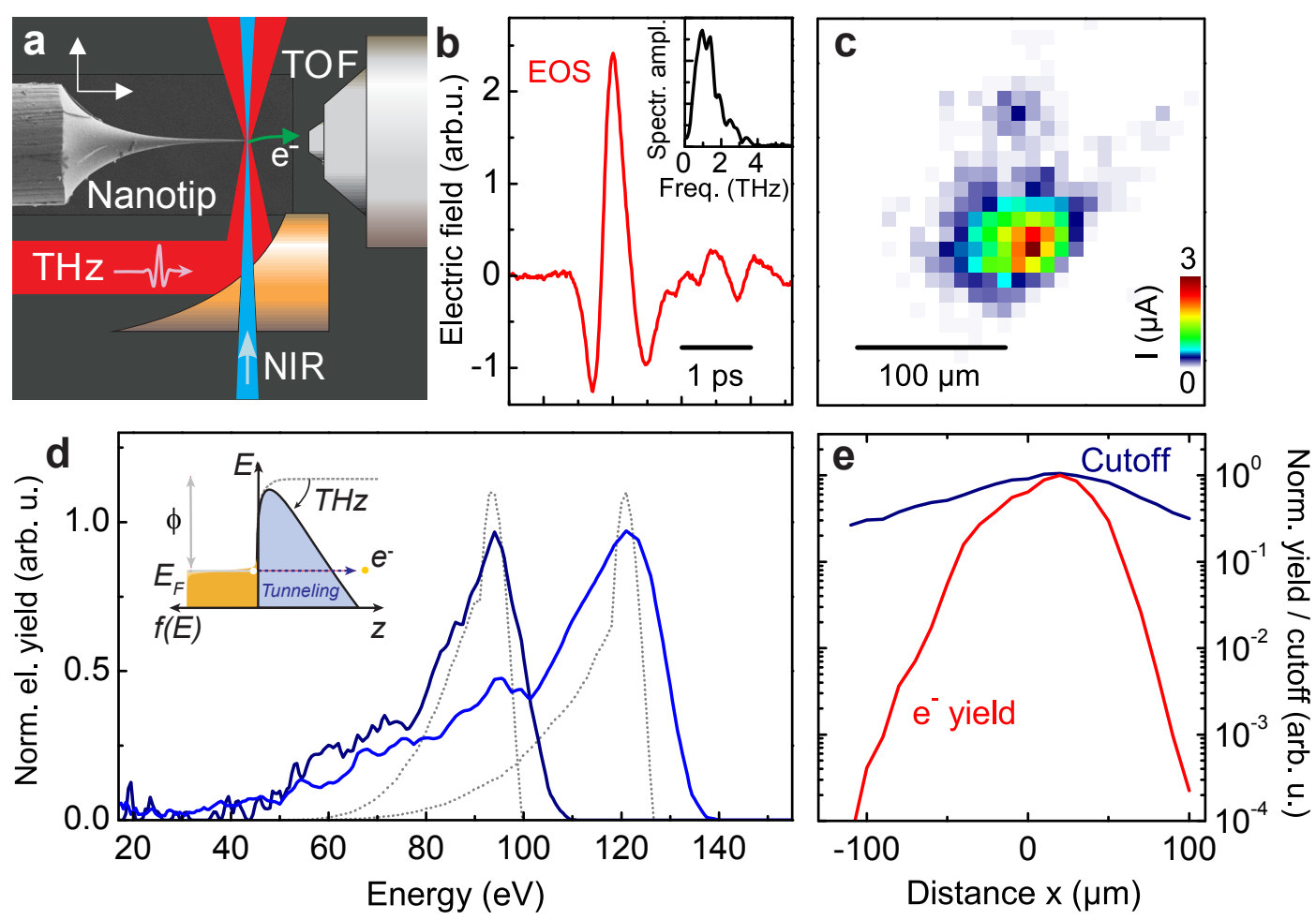

Figure 6.1: a) Schematic of the experiment. b) Typical THz waveform employed, recorded by electro-optic sampling (EOS), and the corresponding spectrum. c) Field emission map acquired by scanning the tip through the $\mathrm{THz}$ focal plane and recording the electron yield (no NIR excitation). d) Electron energy spectra for two local THz field strengths (3.1 V/nm, 5.3 V/nm, $40 \mathrm{~V}$ bias). Dashed lines: numerical simulations. e) Linescan across peak region in c), indicating the tunneling nonlinearity in the electron yield (red). In contrast, the spatial dependence of the kinetic energy cutoff is more extended (blue, energy at $10 \%$ of maximum yield), as it linearly follows the local electric field.

emission, the electrons are accelerated by the locally enhanced $\mathrm{THz}$ field. Recording kinetic energy spectra at every position of the linescan, we can use the electron energy as a measure of the local electric field at the apex, which is governed by the antenna properties of the tip [159]. The linear field-dependence of the kinetic energy cutoff maps the THz focus (blue, Fig. 6.1e), whereas the corresponding electron yield (red) demonstrates a higher localization due to the tunneling nonlinearity [45]. As previously reported [172, 173, 174, 175], focussing plasma-generated THz-radiation results in complex spatio-temporal distributions with higher frequencies in the focal center. In this work, we are primarily interested in sub-cycle dynamics within few-THz excitation, and such transients are found somewhat outside the focal center. Thus, for the following measurements, 
the tip apex is positioned $300 \mu \mathrm{m}$ off-center within the low-frequency region of the beam waist, as characterized by nanotip streaking (see below). The resultant reduction in local peak field is compensated by generating more intense incident pulses.

Figure 6.1d displays recorded time-of-flight spectra for two different $\mathrm{THz}$ pulse energies, revealing peaked energy distributions with sharp cutoffs. The spectral shape results from the nonlinear emission at maximum field strength and subsequent acceleration in the THz-induced momentary potential. Some spectral modulations at intermediate energy (low-energy pedestal) are caused by the spectrometer response, and power fluctuations of the $\mathrm{THz}$ pulses in the range of few percent lead to some additional broadening. Such characteristic quasi-static spectral shapes were also observed in microwave fieldemission [52], resulting from the sub-cycle transition through the mm-sized field in the active resonator gap, but insensitive to apex near-fields. In contrast, strong-field photoemission spectra in the near- and mid-infrared display a maximum electron yield at low energy, a plateau region and a cutoff at higher energies [20, 70, 71, 176]. In comparison with mid-infrared excitation, contributions of lower-energy electrons are reduced at $\mathrm{THz}$ frequencies by reaching even deeper into sub-cycle, field-driven acceleration [20]. The spectral characteristics are reproduced by numerical evaluation of electron tunneling and propagation in the near-field (Fig. 6.1d, dashed lines). The spectra imply ultrafast emission in a temporal window below $100 \mathrm{fs}$ and a local field enhancement of about 200 . We do not detect electron emission for inverted $\mathrm{THz}$ polarity, as the peak field is reduced by a CEP-shift of $\pi$.

We now track the temporal evolution of the THz potential at the tip apex and resolve the onset of $\mathrm{THz}$ field emission by employing a pump-probe scheme with 50-fs near-infrared pulses. Assisted by optical field enhancement at the tungsten tip [177], the short NIR pulse generates photoelectrons localized to the apex [20, 57, 62, 63, 70, 71, 146, 155, 178, 179], which are accelerated in the momentary $\mathrm{THz}$ potential. This near-field streaking technique was introduced in Ref. [21] and yields direct access to the local electric waveform at the apex. Photoelectron spectra as a function of THz-NIR delay represent streaking spectrograms, four of which are shown in Figs. 6.2a-d for increasing THz field strength. The delay-dependent spectrograms exhibit a number of rather specific features, such as the onset of kinetic energy immediately after the suppression of the current at small negative delays (-200 fs in Fig. 6.2b) or the downward shift around -1 ps. A comparison with numerical simulations (Fig. 6.2f) gives quantitative evidence for the actual combination 

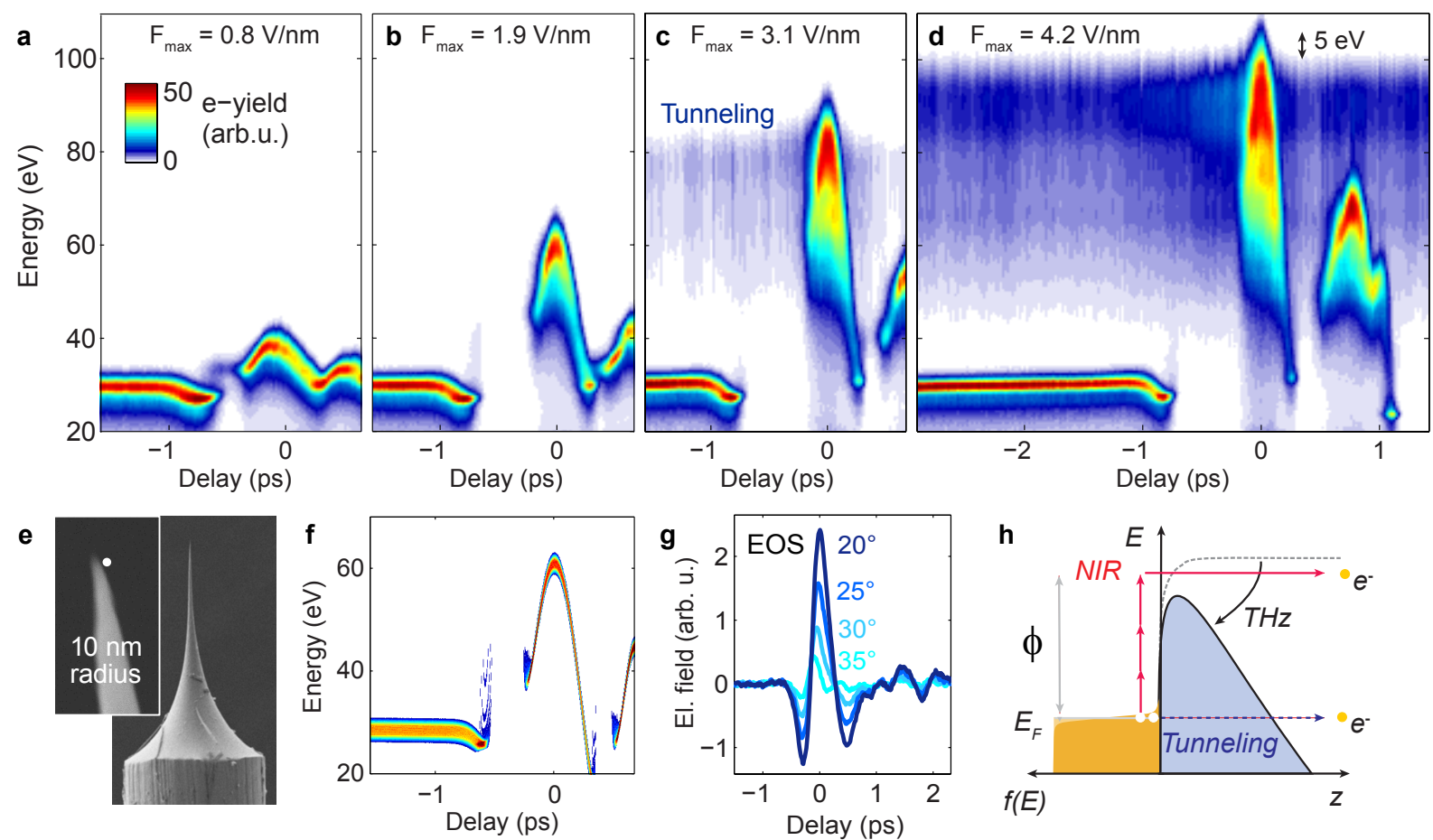

Figure 6.2: a) - d) Streaking spectrograms for increasing THz peak field show the temporal evolution of the near-field and $\mathrm{THz}$ tunneling in $\mathrm{c}, \mathrm{d}$ ). The energy cutoff at zero delay is shifted by $5 \mathrm{eV}$ compared to $\mathrm{THz}$ field emission spectra for delays $<0.5 \mathrm{ps}$. e) Scanning electron micrographs of the nanotip used in the experiments. White circle in the inset has $10 \mathrm{~nm}$ radius. f) Numerical simulation of streaking trace, corresponding to the conditions of the measurement shown in b). g) Electro-optic sampling traces for various BBO angles relative to the angle of maximum THz efficiency. Transients used in the spectrograms a),b),c) and d) correspond to angles of $35^{\circ}, 30^{\circ}, 25^{\circ}$ and $23^{\circ}$, respectively. h) The energy shift between the cutoffs in THz field emission and maximum streaking energy (see d) follows from the different emission processes, which are either over-the-barrier emission at coincident NIR and THz fields, or THz tunneling through the barrier.

of decay length and field strength. Given the known apex dimension of the employed nanostructure and, the field strengths are deduced as the only free parameter to match experiment and simulation. At the lowest local peak field of $0.8 \mathrm{~V} / \mathrm{nm}$ (Fig. 6.2a), we observe energy shifts of the NIR-induced spectra purely arising from acceleration in the instantaneous THz near-field. The electron dynamics in the temporally and spatially varying $\mathrm{THz}$ transient are accurately described within a particle propagation model [21], see for example the simulation in Fig. 6.2f corresponding to the experimental spectrogram in Fig. 6.2b.

For a local peak field strength of $1.9 \mathrm{~V} / \mathrm{nm}$ (Fig. 6.2b), we observe an enhancement in the photoemission yield around the $60-\mathrm{eV}$ streaking maximum, caused by a THz-induced reduction of the work function, known from static fields as the Schottky effect [12]. At a further increase to peak fields of $3.1 \mathrm{~V} / \mathrm{nm}$ and $4.2 \mathrm{~V} / \mathrm{nm}$ (Figs. 6.2c,d), a continuous hori- 
zontal stripe appears around maximum streaking energy, representing THz field emission for all delays. In both spectrograms, this striped feature displays a cutoff that is $5 \mathrm{eV}$ lower than the respective maximum streaking energy (cf. Fig. 6.2d). This shift on the order of the metal work function stems from the different emission processes: NIR-induced electrons are emitted above the barrier into vacuum, whereas field-emitted electrons tunnel at the Fermi energy through the barrier, see Fig. 6.2h. The slightly higher difference of $5 \mathrm{eV}$ compared to the work function of around $4.5 \mathrm{eV}$ may be attributed to residual propagation effects, as the maximum kinetic energy is acquired at emission times slightly before $(<20 \mathrm{fs})$ the peak field, at which tunneling occurs.

In addition to resolving the onset of $\mathrm{THz}$ tunneling, the streaking measurements of Fig. 6.2 effectively represent a sampling of the strength and temporal structure of the $\mathrm{THz}$ near-field ("pump") with NIR photoemission ("probe"). However, it is also possible to exchange the roles of pump and probe pulses: NIR-induced ultrafast carrier dynamics within the tip can be locally probed by using the temporally confined $\mathrm{THz}$ field emission. This is demonstrated at higher NIR intensity, i.e., significantly elevated electronic temperature. Under such conditions, the THz field emission displays a time-dependent signature reaching into negative delays, observed in the spectrogram of Fig. 6.3f (saturated color scale) at high electron energies and delays between 0 and $-1.5 \mathrm{ps}$. The temporal decay of this emission signal measures the relaxation of electronic temperature after NIR excitation, selectively at the apex.

Generally, hot electron dynamics in nanoscopic solids, e.g. in thin films, can be drastically altered by the spatial confinement as compared to the bulk material [180, 181, 182]. The pronounced nonequilibrium electron population generated by single and multiphoton excitation thermalizes to a hot Fermi-Dirac distribution via electron-electron scattering [54, 183], which may be accelerated for small nanoparticles [182]. The size of the nanotip apex approximately equals the optical penetration depth and the range for ballistic transport, resulting in essentially homogeneous heating of the apex electrons. Thus, we regard electron-phonon coupling as the exclusive cooling channel in the first few ps following the homogeneous electronic excitation in the apex. Ballistic and diffusive hot electron transport into the bulk are inhibited in the confined geometry compared to bulk samples. We model this behaviour by calculating the electronic temperature in a twotemperature model [184], Fig. 6.3d, evaluating the THz-induced tunneling for different electronic energies [45]. We employ the experimental excitation parameters, the material constants for bulk tungsten [119] and, because of the nanoscale carrier confinement, do 
a

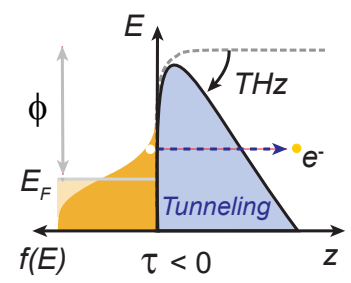

b

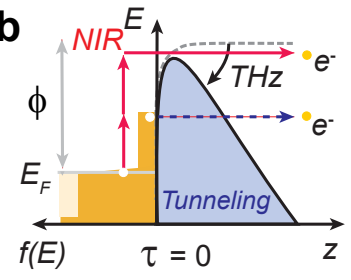

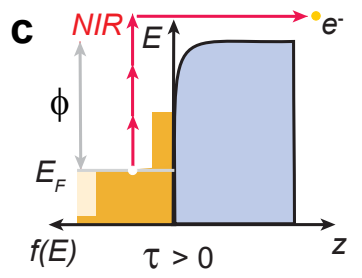

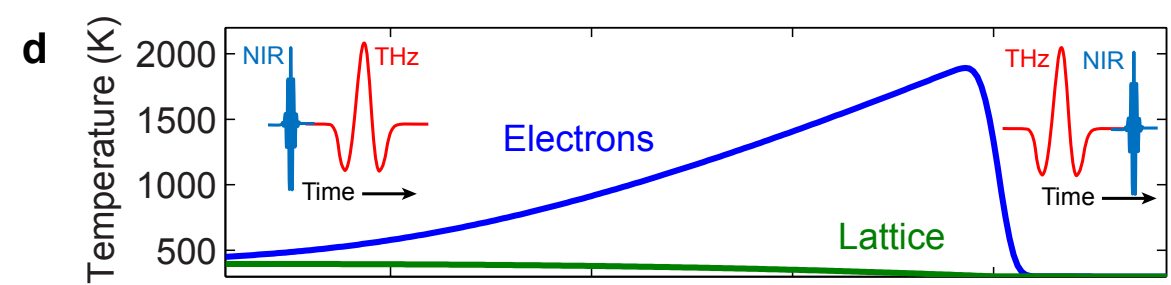

e

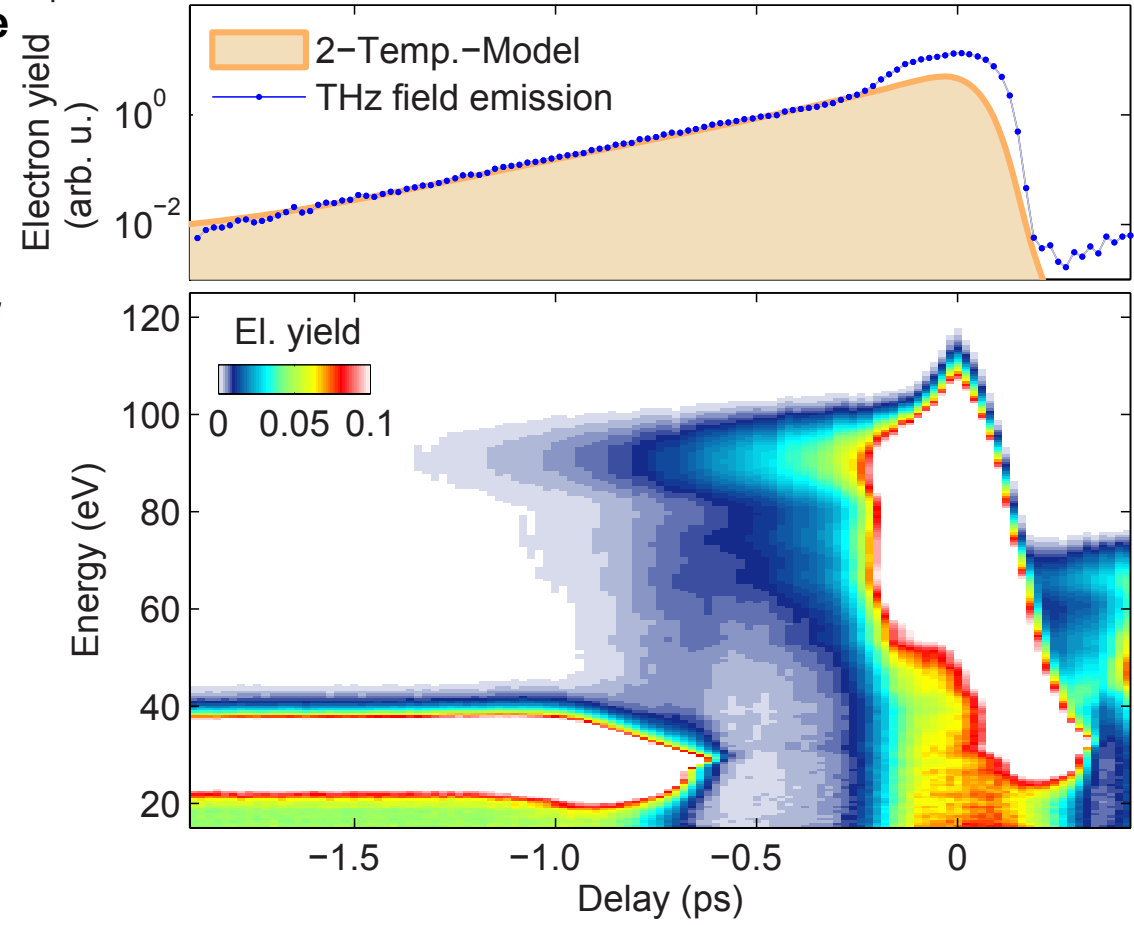

Figure 6.3: Following transient carrier dynamics in the apex via THz field emission. a) For delays $\tau<0 \mathrm{ps}$, the NIR pulse precedes the THz pulse. Here, THz field emission probes the relaxation of the NIR-excited hot carrier distribution. b) At temporal overlap $\tau=0 \mathrm{ps}$, NIR-photoemission is enhanced by the THz-induced Schottky effect. c) For delays $\tau>0$ ps, the NIR pulse succeeds the THz pulse, and the NIR-induced multiphoton photoemission is largely unaffected by the $\mathrm{THz}$ field. d) Electronic and lattice temperatures computed in a two-temperature-model for parameters of the measurement in $\mathrm{f}$ ). e) Transient $\mathrm{THz}$ field emission (blue circles) obtained from spectrogram in $\mathrm{f}$, and prediction from two-temperature model (shaded area). The additional enhancement around zero delay is caused by the Schottky effect. f) Spectrogram for $350 \mathrm{GW} / \mathrm{cm}^{2}$ incident NIR intensity, displayed in a saturated colormap to highlight the hot electron dynamics at high kinetic energies. 

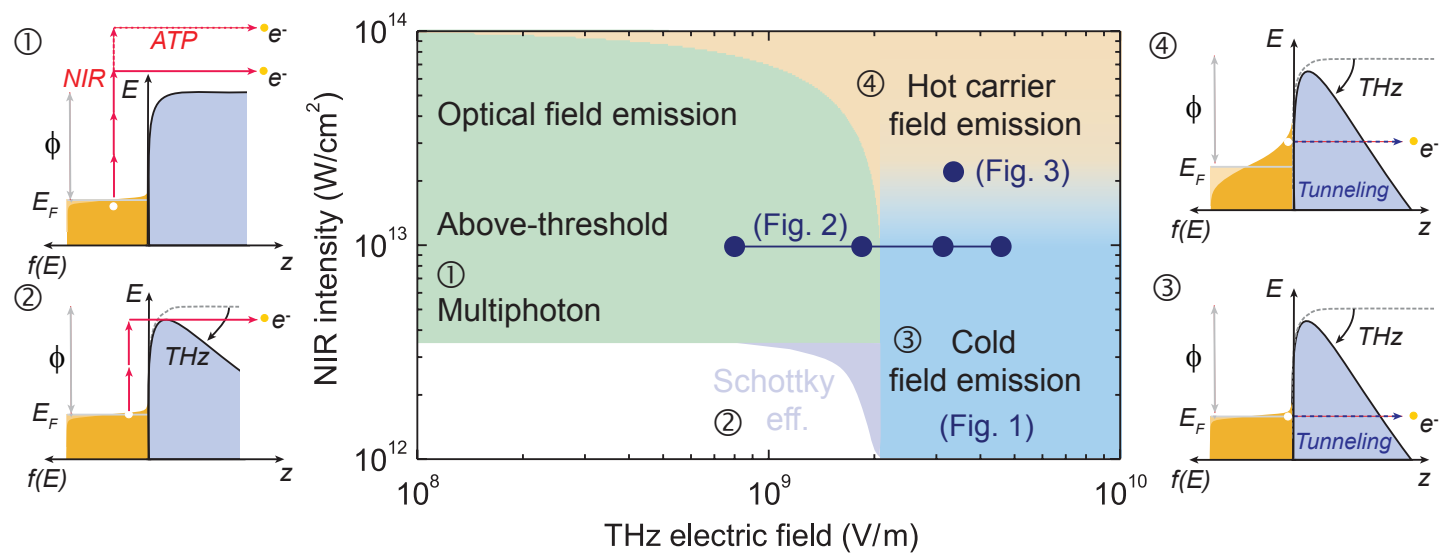

Figure 6.4: Map of predominant emission regimes for various local NIR intensities and THz fields, referring to conditions in this work (labeled by Figure numbers) and prior works in the absence of $\mathrm{THz}$ fields (references given in main text). In particular, the present work demonstrates nondestructive access to cold field emission (Schematic 3), nanotip streaking (Fig. 6.2) and Schottky enhancement (Schematic 2), as well as hot carrier field emission at THz-frequencies (Schematic 4).

not include ballistic or diffusive spatial energy transport. The electron-phonon coupling constant of bulk tungsten is expected to be unaffected in the nanoscopic geometry [185]. This simplified model accurately reproduces the experimental near-exponential decay of the electron yield (plotted on a logarithmic vertical scale) with a (1/e)-decay time of $0.3 \mathrm{ps}$ (Fig. 6.3e). The corresponding electronic temperature (Fig. 6.3d) decays largely linearly in the first picosecond, and a decay of the temperature rise to a fraction of (1/e) is found within 1.2 ps.

The observed difference to sub-ps exponential cooling at bulk surfaces found in nonlinear photoemission studies [186] and recent transient reflectivity measurements [187] indicates the impact of nanoscale confinement on the hot electron dynamics. Previously studied cooling curves in gold films for varying thickness [180, 181] identify a linear temperature decrease and slower cooling as hallmarks of dynamics with carrier confinement and predominant cooling via electron-phonon coupling. Thus, our linear time dependence in temperature and slightly longer cooling times compared to bulk studies demonstrate that ballistic and diffusive hot carrier transport into the volume do not present significant contributions.

In conclusion, Figure 6.4 reviews the measurements in the experimental parameter 
space spanned by the NIR intensity and the THz field strength, and classifies the dominant emission regimes. At NIR excitation alone, nonlinear multiphoton photoemission is achieved for locally enhanced intensities below $6 \mathrm{TW} / \mathrm{cm}^{2}$ for gold tips [60], and strong-field effects are observed for both gold and tungsten nanotips around $20 \mathrm{TW} / \mathrm{cm}^{2}$ $[20,60,63,70,71]$. Damage is reported at several $10 \mathrm{TW} / \mathrm{cm}^{2}$. Under intense NIR illumination, the transition to $\mathrm{THz}$ field emission is mediated by photon-assisted or thermallyenhanced field emission, in analogy to NIR-photoemission studies at nanotips with high static bias potentials $[62,188]$. We experimentally trace the transition to $\mathrm{THz}$ field emission at moderate NIR fields, as presented in Fig. 6.2. For higher NIR intensities, THzinduced emission is enhanced by tunneling of hot electrons (Fig. 6.3). In contrast, THz transients alone enable ultrafast cold field emission for local fields above $2 \mathrm{~V} / \mathrm{nm}$, reached in the near-field of the nanotip (Fig. 6.1).

More generally, this highly-nonlinear emission allows for local pump-probe schemes to selectively access the carrier dynamics in confined metallic or semiconducting nanostructures and enables novel schemes to probe, bias and control material systems on ultrafast timescales.

\section{Acknowledgment}

We thank G. Storeck and K. E. Echternkamp for technical support, and D. R. Solli, S. Schäfer and M. Sivis for helpful discussions. We gratefully acknowledge financial support by the Deutsche Forschungsgemeinschaft (DFG-SPP 1391 'Ultrafast Nanooptics' and $\mathrm{ZuK} 45 / 1)$.

\section{Author contributions}

The study was conceived by GH, LW and CR. The experiments were carried out by GH, with contributions from LW. GH implemented the numerical model for spectrogram simulations and for the evaluation of the transient hot electron tunneling. $\mathrm{GH}$ analyzed the data, with contributions from LW and CR. All authors discussed the findings in detail. GH drafted the paper, which was revised by all authors. 


\section{Chapter 7}

\section{Discussion}

In the following, we highlight the main results of the preceding three articles. Related and complementary aspects are discussed in perspective, and several concepts which bear the potential for future technological and scientific applications are presented in more detail.

\subsection{Light-electron interaction in optical near-fields}

In Chapter 4, the field enhancement at the apex of gold nanotips is demonstrated to allow for localized electron emission at moderate intensities over a broad wavelength range from $800 \mathrm{~nm}$ to above $8 \mu \mathrm{m}$. The mid-IR spectral range provides access to the strongfield regime at Keldysh-parameters below $\gamma=0.1$ without sample damage. Experimental data and numerical simulations prove the validity of a semi-classical two-step model for strong-field photoemission from metals at long wavelengths. The near-field confinement at the nanotip apex is demonstrated to result in the breakdown of ponderomotive energy scalings, which are usually observed in atomic strong-field ionization. Specifically, we establish conditions under which electrons escape the driving field within a fraction of the optical half-cycle. The correct description relies on including the near-field profile into "Simple-man's" calculations, as the dynamics are based the coupling of femtosecond electronic motion to the nanoscopic near-field distribution.

In analogy to the Keldysh parameter $\gamma$ for the characterization of ionization [36] in intense light fields, the corresponding parameter $\delta$ was introduced for the post-ionization dynamics. Thus, the regimes of ponderomotive and sub-cycle electron acceleration in this second step of the strong-field interaction are distinguished. Whereas the Keldyshparameter relates the driving frequency to the tunneling rate, the "spatial" adiabaticity- 


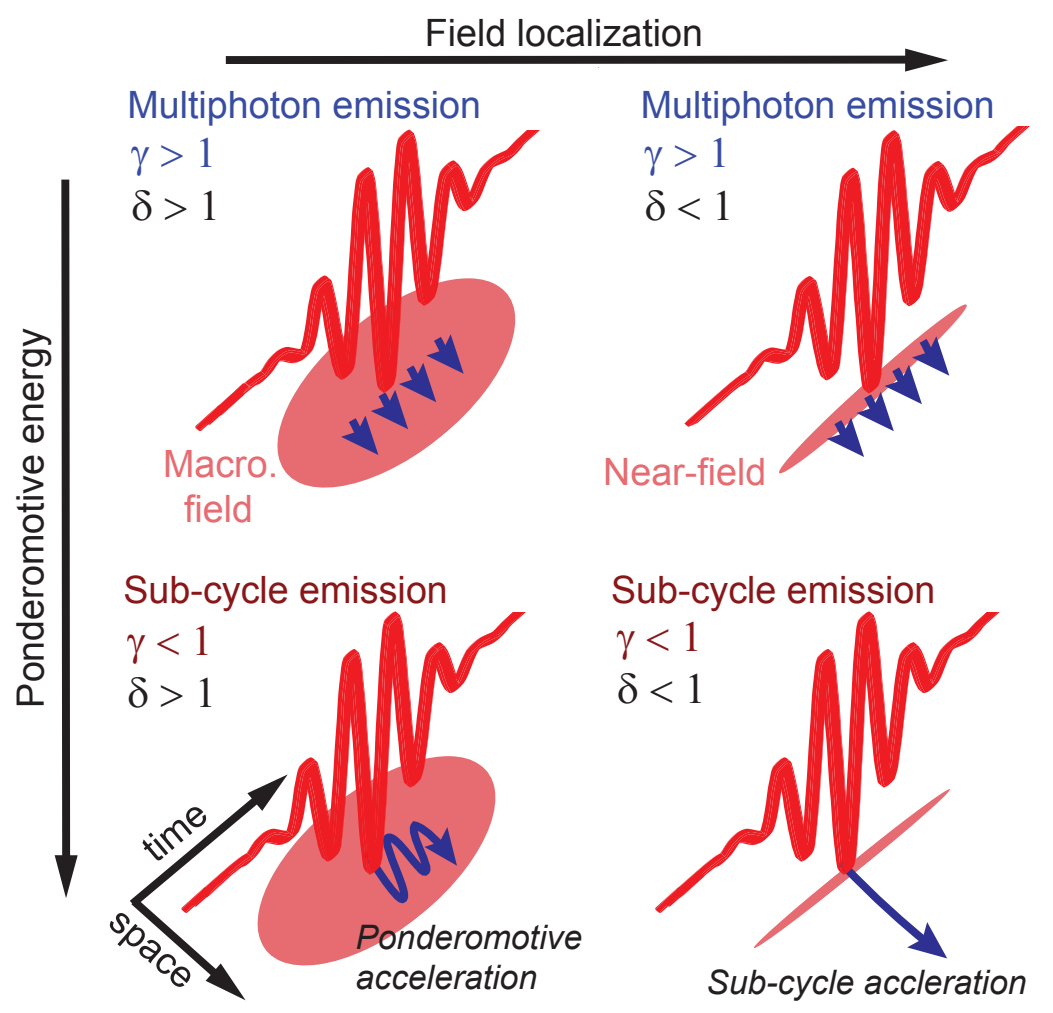

Figure 7.1: Light-electron interactions, given by the transition from multiphoton to strong-field photoemission and by the localization of the driving field (light: red, electron: blue). In the multiphoton regime (top), the emission is governed by the intensity envelope, and ponderomotive acceleration is negligible. In the strong-field regime (bottom), the emission can be temporally confined to the peak of a single field oscillation. Driven by a macroscopic electric field, the electron oscillates and experiences a ponderomotive acceleration. The field localization enables a regime of sub-cycle emission, acceleration, and escape from the driving field at the peak of the electric oscillation.

parameter relates the driving frequency to the electron's escape time from the driving field. In both cases, parameter values far below unity indicate dynamics that are governed by the momentary electric field. Such field-driven emission and acceleration conditions are generally achieved for high fields at low frequencies.

Within a certain range, the regimes of emission and acceleration are independently adjustable, namely the tunneling rate via the work function and the near-field escape time via the decay-length $l_{F}$. The ratio between both parameters is frequency dependent,

$$
\frac{\delta}{\gamma}=l_{F} \omega \sqrt{\frac{m_{e}}{2 \Phi}}
$$


opening a set of distinct conditions of light-electron interactions, summarized in Figure 7.1.

Few-cycle excitation in the strong-field photoemission regime at $\gamma<1$ allows for electron emission governed by the electric field and confined to a single oscillation peak. For a highly-confined field below the diffraction limit, the emitted charge can be ejected from the oscillatory force within a fraction of the half-cycle. Such dynamics enable minimal temporal and energetic dispersion due to the rapid acceleration and suppressed rescattering.

The use of Terahertz radiation is a significant extension of the wavelength-dependent study into the far-infrared up to a wavelength of $\lambda=300 \mu \mathrm{m}$. At sufficient local fields, we access the purely field-driven emission and acceleration regimes at $\delta, \gamma<1$ and demonstrate $\mathrm{THz}$-induced field emission, as presented in Chapter 6 . We observe a characteristic narrowing of the energy spectra, resulting from the emission and acceleration at the peak of a single-cycle transient. Comparing mid-infrared emission spectra at comparable fields, we find an effective inversion of the spectral shape for increasing wavelength, turning into a single high-energy peak at Terahertz frequencies.

The investigation of the near-field acceleration process is extended by two-color experiments with phase-stable, single-cycle $\mathrm{THz}$ pulses $^{1}$ in Chapter 5, allowing for the phaseresolved mapping of the photoelectron dynamics via streaking spectrograms. We find that high local THz-fields $(F \approx 1 \mathrm{~V} / \mathrm{nm})$ at sharp nanotips $\left(l_{F} \approx 10 \mathrm{~nm}\right)$ result in quasiinstantaneous streaking conditions, permitting the time-resolved detection of the local THz-near-field at the nanotip apex. The comparison to the incident THz-transient reveals waveform modulations due to the electro-magnetic response of the semi-infinite antenna structure of the nanotip. Microscopic differences in the nanotip geometry can significantly impact the near-field response, as observed over the course of the streaking experiments. The impact of a retarded carrier accumulation due to optical and electronic propagation effects onto the local field enhancement may be disentangled by the application of nearfield streaking onto different metallic nanotips. In particular, the process of adiabatic nanofocussing in conical structures could be resolved in the time-domain [189, 190, 191]. A promising extension to different samples includes, for instance, the investigation of semiconductor nanostructures [147], or resonant metallic dipole [192] and split-ring antennas [193]. The precise characterization of the local electric fields is substantial for the

\footnotetext{
${ }^{1}$ The electric waveform of non-CEP-stabilized laser sources drifts from pulse to pulse. The averaging over multiple pulses generally results in a loss of the CEP-sensitive information.
} 

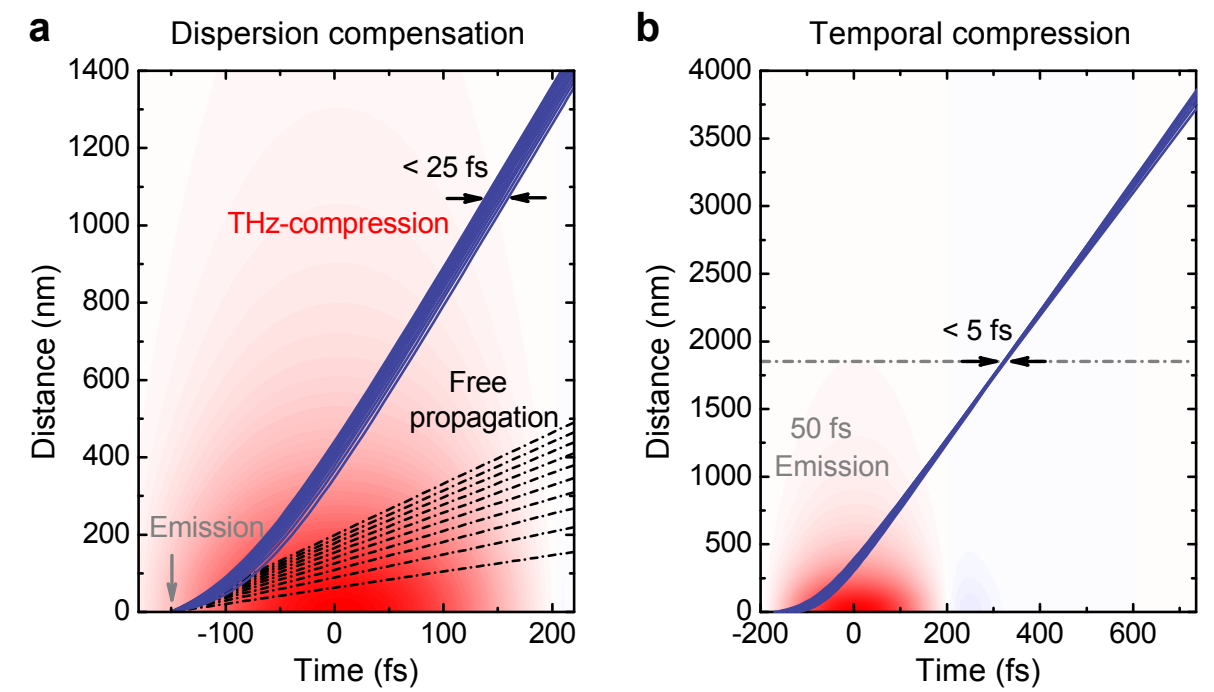

Figure 7.2: a) Simulation of the THz-induced spectral compression of an instantaneously emitted photoelectron distribution of $5 \mathrm{eV}$ width (initial energies equally spaced from $0.5-5 \mathrm{eV}$ ). The THz peak field is $0.2 \mathrm{~V} / \mathrm{nm}$ and the decay length $200 \mathrm{~nm}$, color-coded in the background. In this example, the near-field interaction facilitates the dispersion-free propagation of 25 -fs electron pulses. For comparison, free dispersive propagation without $\mathrm{THz}$ field (dashed trajectories). b) For temporal compression, the time-dependent THz-field can induce a pre-chirp onto an initially narrow-energetic electron pulse of 50-fs duration. Propagation and the accompanied dispersion results in the compression to a sub-5 fs electron pulse at an adjustable distance from the electron source.

investigation of field-driven processes in nano-optical systems. This scheme allows for the time-domain spectroscopy of the high-frequency response of electrical circuit components extending to the THz-range — at frequencies not accessible with state-of-the-art microelectronics.

In the transition region between sub-cycle and ponderomotive acceleration $(\delta \approx 1)$, the electron dynamics are found to sensitively depend on the temporal derivative of the momentary field, on the electron excess energy and the spatial near-field profile. This spatiotemporal coupling of the electron motion to the driving near-field opens up the possibility to tailor electron trajectories directly at the emission site on femtosecond timescales with the application of increasing the temporal resolution of ultrafast imaging [13] and diffraction [12] instruments.

In a recent implementation of time-resolved low-energy electron diffraction (LEED) [14], the temporal resolution was limited to few-ps, mainly by the energetic dispersion of the electron pulse upon propagation. Alongside a means of minimizing the excess energy 
spread by adjusting the photon energy to the work function [12], the propagation in a superposed THz-near-field allows for a tunable dispersion compensation, as illustrated in Figure 7.2a. Initially lower kinetic energies are compensated by more effective acceleration, yielding energetically-compressed electron pulses with only few 10-fs duration at the sample position, assuming low electron numbers and negligible space-charge repulsion.

Alternatively, a temporal compression to extremely short few-fs electron pulses at a predefined distance from the source is attainable via the THz near-field. For instance, electrons emitted during a 50-fs laser excitation experience a balanced pre-chirp which depends on the emission time, reaching the distant sample position coincidentally, see Figure 7.2b. This scheme is ideally suitable for miniaturized optical-pump / electronprobe experiments with the electron source in close proximity to the sample under investigation, as in Ref. [194]. In order to reach the minimal electron pulse durations, spacecharge repulsion [141, 142, 143, 144, 162], and the combined effects of the emission time spread and the excess energy dispersion have to be studied in more detail, e.g., by three-dimensional simulations, including Coulomb-interaction between emitted electrons. Future experiments to characterize the pulse-widths of temporally compressed electron pulses could be based on ponderomotive acceleration [195], the transient electric fieldeffect [196, 197] or the PINEM-effect [198, 199].

High static acceleration of such low-dispersive electron pulses onto metal targets may provide flexible and compact designs for ultrashort X-ray sources [200], which commonly employ laser-plasma interactions $[15,16,17]$. Such sources enable ultrafast X-ray diffraction [201] and biomedical imaging, allowing for increased sensitivity and reduced X-ray doses by temporal-gating [202, 203]. Significant current-scaling may be achieved with field-emitter arrays, an approach currently being pursued, e.g., at the Paul-ScherrerInstitute [204], and directly connected to the development of electron guns for novel freeelectron X-ray lasers ${ }^{2}$ [205, 206].

\subsection{THz near-field streak camera}

The presented schemes of electron pulse compression are based on electron emission into temporally rising $\mathrm{THz}$ near-fields; however, the emission into a falling slope of the electric force facilitates the enhancement of initially small temporal or energetic variations in

\footnotetext{
${ }^{2}$ First results on THz-acceleration of optically-induced charges from flat photocathodes were reported in Ref. [168].
} 

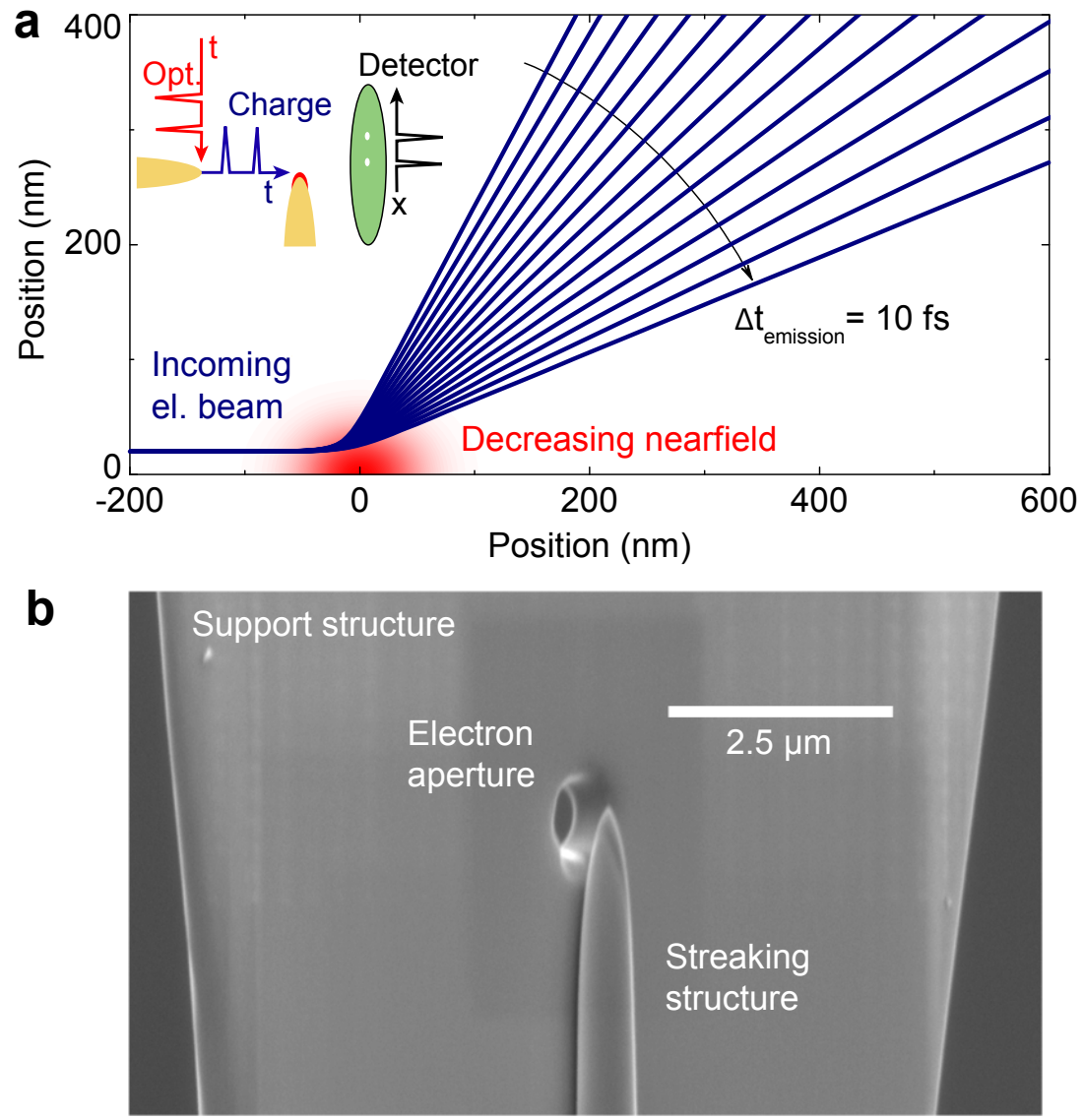

Figure 7.3: a) Schematic of a THz near-field streak camera. Inset: Ultrafast optical signals induce photoelectrons at a photocathode or suitable nanostructure, the emitted charges are streaked in the near-field and recorded on a spatially-resolving electron detector. Graph: Classical electron trajectories in the near-field streak camera illustrate the angular dispersion of electrons, equally-separated by $10 \mathrm{fs}$. b) Scanning electron micrograph of a prototype streaking structure: Electrons are generated at a nanotip on the backside of the structure and are collimated in the electrostatic potential between the emitting tip and the electron aperture. The streaking structure is positioned with respect to the aperture and electrically connected or isolated to the support. Image: courtesy of K. E. Echternkamp.

the photoelectron distribution. This effect could be exploited for a novel streak-camera scheme, combining various features of attosecond-, nanoplasmonic- and THz-streaking. The principle design is based on two nanostructures: the first converts photons into photoelectrons, and the second provides the streaking near-field. A simplified scheme is sketched in Figure 7.3 to illustrate the specific electron interaction with the streaking nearfield. The key technical features are as follows: 
- Electron emission from nanotips significantly reduces the large spread of initial momenta, typical for macroscopic photocathodes, and increases the temporal resolution [59].

- Compared to electro-optical streaking, all-optical streaking allows for the nearperfect synchronization between trigger and streaking fields, extremely high temporal resolution and for detection at Megahertz repetition rates.

- Compared to attosecond streaking, all-optical streaking at THz-frequencies supports few-fs temporal resolution, while covering a temporal detection window exceeding $200 \mathrm{fs}$, allowing to capture relevant time-scales of fast molecular and solid state dynamics [4].

- Photo-generated electrons are streaked at a second nanostructure by the deflection of the trajectories in the streaking near-field. The key feature is the largely enhanced sensitivity of the electron near-field acceleration with regard to the arrival time. Instead of encoding the temporal information via subtle energetic differences - the usual case of all-optical streaking — the time is projected onto deflection angles. This property largely simplifies the instrument requirements since photoelectron spectrometers are not needed. Corresponding to a true streak "camera", the deflection is captured as a spatial streaking trace on an imaging electron detector. This streaking unit could be easily fabricated as a cathode ray tube of few-mm dimension.

- The high THz field-enhancement at metallic nanostructures of above 100 may allow the usage of compact commercial THz sources, based on fiber lasers and photoconductive switches, thus enabling the realization of complete streaking devices of extremely compact dimensions.

- Coupling fast electronic signals to the streaking structure and employing singleelectron detector arrays may enable a new type of analog-to-digital converters (ADC) with highly increased sampling rates above $100 \mathrm{Gs} / \mathrm{s}$. Similar ideas are pursued, for instance, in the Pease group at Stanford University, USA [207].

A related concept - based on split-ring resonators - was very recently proposed, and may ease the alignment and data post-processing due to a principally higher symmetry of the 
near-field distribution within the resonator gap [208]. The three-dimensional electromagnetic and particle propagation simulations including electron repulsion yields temporal resolutions close to $1 \mathrm{fs}$ for realistic operation parameters.

The presented schemes demonstrate new perspectives for ultrafast vacuum microelectronics [209, 210], as the near-field control of propagating electron pulses enables new functionalities and switching speeds unattainable in conventional microelectronics. As they are not limited by the dielectric material response, "vacuum-electronics" could be reinvented, enabled by the combined influence of modern nanofabrication tools and ultrafast optics. Specifically, the demonstrated near-field acceleration could enable a rise of the electronic gating rate by the reduction of the interaction time of the electrons with the driving field. State-of-the-art 3D-nanofabrication capabilities and the onset of electronic tunneling [211] set a realistic minimum size of the active nanostructures and the respective near-fields on the order of $l=5 \mathrm{~nm}$. Assuming an initial electron kinetic energy of, e.g., $E=3 \mathrm{eV}$, which implies a velocity of $1 \mathrm{~nm} / \mathrm{fs}$, the interaction time in the transit through the near-field is only $5 \mathrm{fs}$, corresponding to possible gating rates of $200 \mathrm{THz}$.

At this point, one might wonder if concepts from ultrafast optics have the potential to emerge as the future building blocks of advanced electronic devices, ultimately replacing conventional microelectronics. Yet, it should be noted that the term "transistor" is frequently encountered in the literature as a synonym for "switching" in an over-simplified context. Naturally, the rapid deflection of electrons does not constitute the function of an electronic transistor. As pointed out for "optical transistors" [212], many proposed ultrafast transistor schemes do not meet basic requirements of signal cascadability, sufficient gain and level restoration to form practical logic units. This generally holds for state-ofthe-art schemes to optically steer electronic motion with attosecond pulses which - although outreaching transistor speeds by orders of magnitudes - typically do not allow for large-scale integration. However, one may still speculate that the presented near-field approaches may potentially be combined with sub-diffraction light propagation via surface plasmons-polaritons and coherent control $[213,214]$ to enable more complex schemes for ultrafast electro-optical logic units. Limitations due to high metallic absorption at optical frequencies could be efficiently bypassed in the mid-IR [215, 216], still the miniaturized detection of few electron bunches presents a severe technological challenge. 


\subsection{Field emission as an ultrafast probe}

The sub-100 fs temporal window of THz field emission establishes a novel pump-probe scheme to resolve ultrafast carrier dynamics on the nanoscale. As presented in Chapter 6, the setup corresponds to the above near-field-streaking scheme, only here, the roles of the pump and probe pulses are interchanged. A preceding pump, e.g., by UV, visible or NIR-pulses, triggers a transient excitation in the sample, which is probed via the delayed THz-induced field emission. As a result of the emission nonlinearity, the detected signal originates exclusively from the apex of the tip. Compared to widely employed twophoton photoemission spectroscopy, here, the macroscopic surface signal is effectively suppressed, and only the electron populations in nanoscale protrusions are accessed in the far-field.

In a first application, we investigated the impact of NIR-illumination onto the apex electron population by a NIR-pump / THz-probe experiment. For high NIR-intensities, we observe the generation of NIR-induced transient hot-electron distributions. The timeresolved cooling dynamics indicated heat confinement due to the apex geometry, as reported similarly for $20 \mathrm{~nm}$-thin metal films [180, 181]. Further studies are required to elucidate the impact of the apex diameter and the shaft angle. Electron-phonon coupling is regarded as the dominant cooling channel for sharp nanotips; thus, we expect this scheme to provide access to the electron-phonon interactions in different nanostructures, geometries and mechanisms in more complex materials. Scaling the pump-probe scheme to higher frequencies may enable the tracking of non-equilibrium electronic excitations and transient electron-electron dynamics on 10-100 fs timescales [54].

Moreover, the time-resolved access to the local electron temperature at the apex of nanotips is of crucial significance for the development of field ion microscopes (FIM) for ultrafast studies [217]. Being the first microscope with atomic resolution [218], the application of intense $\mathrm{THz}$ electric fields may open up ultrafast modalities for singleatomic imaging. 


\section{Chapter 8}

\section{Appendix}

\subsection{Notations and Abbreviations}

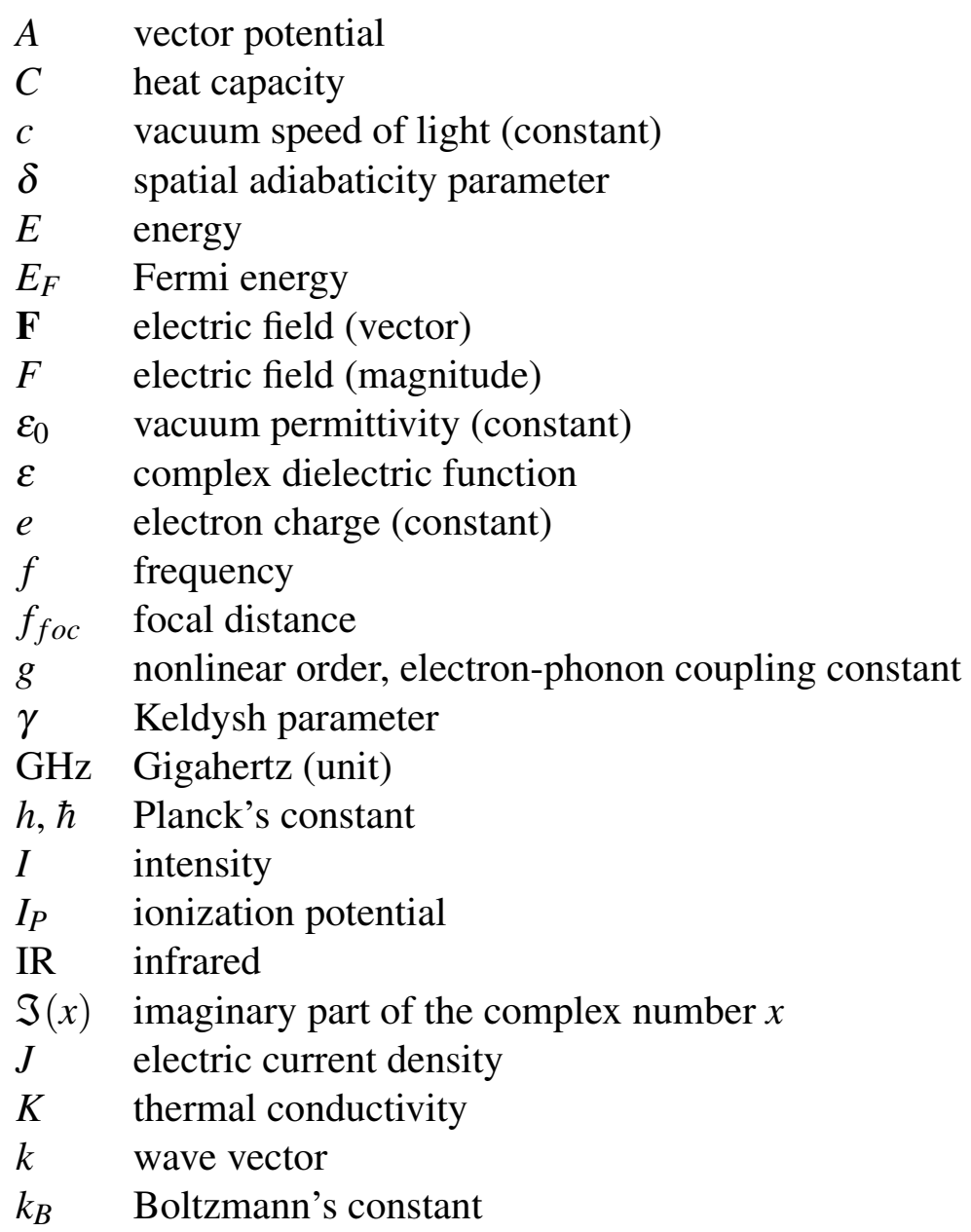




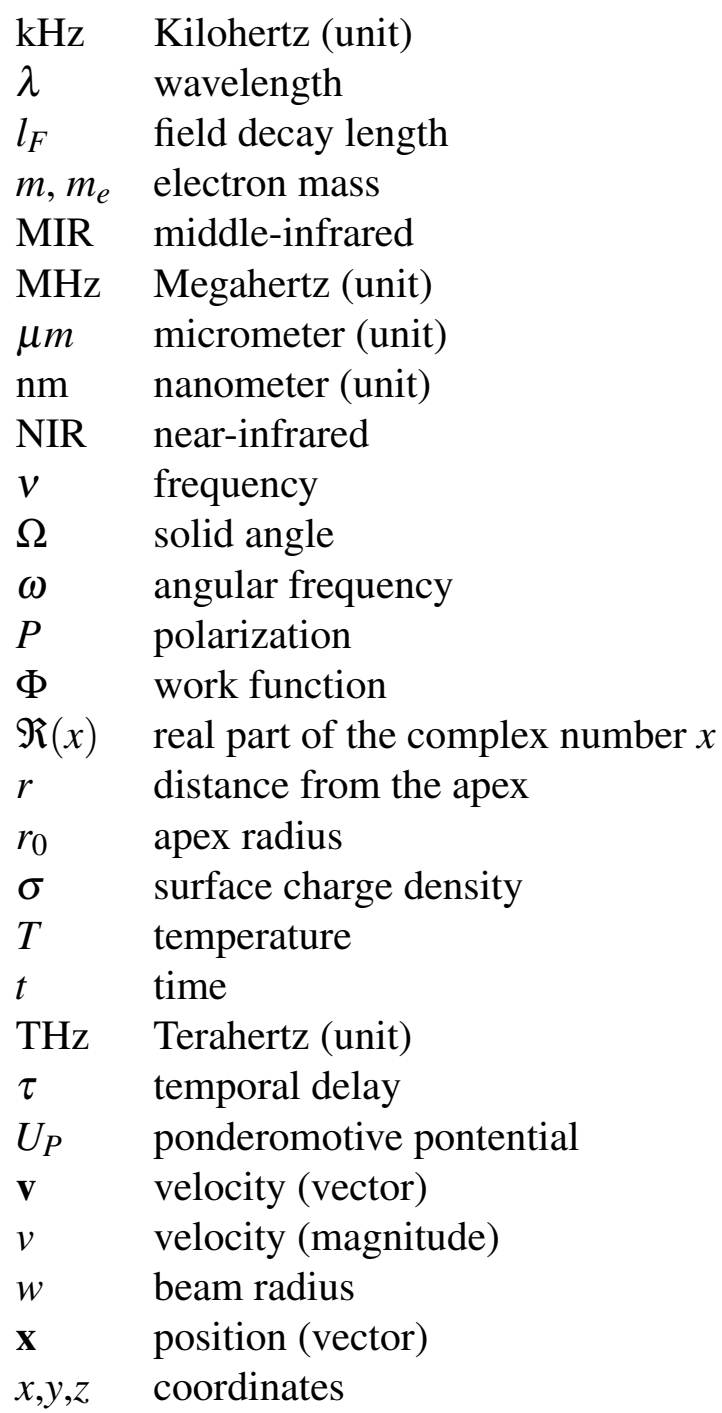

\subsection{Derivation of linear carrier cooling in the absence of transport}

At metals, intense ultrafast illumination may generate local electronic excitations at rates exceeding the thermal equilibration with the lattice system. Initially, femtosecond NIRexcitation instantaneously creates a non-thermal population via direct and multiphoton absorption, illustrated in Fig. 8.1 [54, 62]. Electron-electron scattering is increased above the Fermi-energy and results in a rapid thermalization to a collective electron temperature on timescales of several $10 \mathrm{fs}$. The low electronic heat capacity typically results in high temperatures of several $1000 \mathrm{~K}$. Thermal relaxation is provided via ballistic and diffusive hot electron transport into the bulk material and via energy relaxation to the lattice sub- 


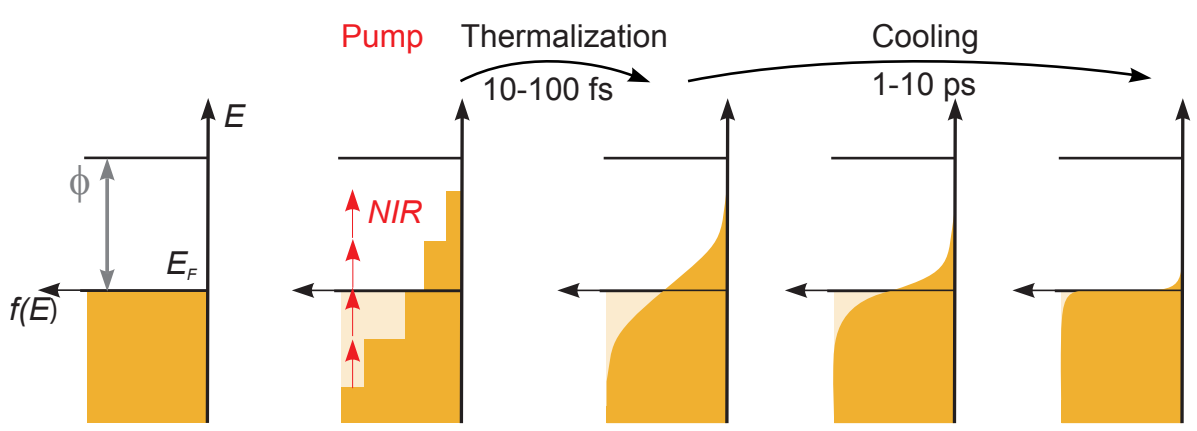

Figure 8.1: Intense ultrashort NIR pump pulses generate non-thermal electronic excitations. Thermalization to a Fermi-Dirac distribution occurs via electron-electron scattering within several $10 \mathrm{fs}$. The hot distribution cools via electron-phonon coupling to the lattice on a timescale of several ps.

system. The dynamics are described in the two-temperature model of Anisimov [184]. Based on Ref. [181], the following adaption for confined nanoscopic structures provides a simplified analytical description for the cooling dynamics, which are experimentally and numerically studied in Chapter 6. The temperature evolution of the electron $T_{e}$ and the lattice sub-systems $T_{L}$ are described via the two differential equations

$$
\begin{array}{cc}
C_{e}\left(T_{e}\right) \frac{\partial T_{e}}{\partial t}= & \frac{\partial}{\partial z}\left(K_{e} \frac{\partial}{\partial z} T_{e}\right)-g \cdot\left(T_{e}-T_{L}\right)+P_{a}(\mathbf{x}, t) \\
C_{L}\left(T_{L}\right) \frac{\partial T_{L}}{\partial t}= & g \cdot\left(T_{e}-T_{L}\right) .
\end{array}
$$

$C_{e}, C_{L}$ are the heat capacities of electrons and lattice, respectively. The temperature dependence of $C_{e}$ is given by the linear expansion $C_{e}\left(T_{e}\right)=\gamma \cdot T_{e} . K_{e}$ is the electronic thermal conductivity, $g$ the electron-phonon coupling term and $P_{a}(\mathbf{x}, t)$ the absorbed power per volume.

In a spatially confined, homogeneously heated nanostructure, ballistic and diffusive transport are inhibited, eliminating the spatial temperature gradient $\frac{\partial}{\partial z}\left(K_{e} \frac{\partial}{\partial z} T_{e}\right)=0$. In addition, the temperature of the initially cold lattice is neglected in the first few ps. An approximation of the decay from the maximum electronic temperature $T_{e, \max }$ after excitation $\left(P_{a}=0\right)$ to the electron-lattice equilibrium temperature $T_{e 0}$ is provided via integration of the simplified equation [181], resulting in:

$$
T_{e}(t)=T_{e, \max }-\frac{g \cdot\left(1-T_{e 0} / T_{e, \max }\right)}{\gamma} \cdot t
$$


In a physical picture, this linear temperature decay results from the fact that the decreasing electron-lattice heat transfer upon cooling is compensated by a (in the first order) equally decreasing electronic heat capacity. This ballance only holds under the absence of heat transport and was previously observed for cooling in nanoscopically confined structures, e.g., thin films and nanoparticles. The resultant cooling rate is increased compared to the exponential decay in bulk material $[180,181,219,220]$. 


\section{Bibliography}

[1] Holzwarth, R., Udem, T., Hänsch, T. W., Knight, J. C., Wadsworth, W. J., and Russell, P. S. J. Optical Frequency Synthesizer for Precision Spectroscopy. Phys. Rev. Lett., 85:2264-2267, 2000. Cited on p. 1

[2] Goulielmakis, E., Schultze, M., Hofstetter, M., Yakovlev, V. S., Gagnon, J., Uiberacker, M., Aquila, A. L., Gullikson, E., Attwood, D. T., Kienberger, R., et al. Single-cycle nonlinear optics. Science, 320(5883):1614-1617, 2008. Cited on p. 1,38

[3] Mourou, G. A., Tajima, T., and Bulanov, S. V. Optics in the relativistic regime. Reviews of Modern Physics, 78(2):309, 2006. Cited on p. 1, 19

[4] Krausz, F. and Ivanov, M. Attosecond physics. Rev. Mod. Phys., 81:163-234, 2009. Cited on p. 1, 37, 38, 41, 42, 60, 63, 89

[5] Van den Heuvell, H. V. L. and Muller, H. Multiphoton Processes. Cambridge Studies in Modern Optics, 25, 1988. Cited on p. 1, 13

[6] Corkum, P. B. Plasma perspective on strong field multiphoton ionization. Phys. Rev. Lett., 71(13):1994-, September 1993. Cited on p. 1, 13, 26, 41, 42

[7] Paulus, G. G., Becker, W., and Walther, H. Classical rescattering effects in twocolor above-threshold ionization. Phys. Rev. A, 52(5):4043-4053, 1995. Cited on p. $1,13,41,42,46,48$

[8] Dürig, U., Pohl, D., and Rohner, F. Near-field optical-scanning microscopy. Journal of applied physics, 59(10):3318-3327, 1986. Cited on p. 1

[9] Novotny, L. and Van Hulst, N. Antennas for light. Nature Photonics, 5(2):83-90, 2011. Cited on p. 1 
[10] Anderson, A., Deryckx, K. S., Xu, X. G., Steinmeyer, G., and Raschke, M. B. FewFemtosecond Plasmon Dephasing of a Single Metallic Nanostructure from Optical Response Function Reconstruction by Interferometric Frequency Resolved Optical Gating. Nano Letters, 10(7):2519-2524, 2010. Cited on p. 1, 61

[11] Goulielmakis, E., Yakovlev, V. S., Cavalieri, A. L., Uiberacker, M., Pervak, V., Apolonski, A., Kienberger, R., Kleineberg, U., and Krausz, F. Attosecond Control and Measurement: Lightwave Electronics. Science, 317(5839):769-775, 2007. Cited on p. 1, 60

[12] Dantus, M., Kim, S. B., Williamson, J. C., and Zewail, A. H. Ultrafast electron diffraction. Experimental time resolution and applications. The Journal of Physical Chemistry, 98(11):2782-2796, 1994. Cited on p. 2, 16, 86, 87

[13] Zewail, A. H. Four-dimensional electron microscopy. Science, 328(5975):187193, 2010. Cited on p. 2, 16, 86

[14] Gulde, M., Schweda, S., Storeck, G., Maiti, M., Yu, H. K., Wodtke, A. M., Schäfer, S., and Ropers, C. Ultrafast low-energy electron diffraction in transmission resolves polymer/graphene superstructure dynamics. Science, 345(6193):200-204, 2014. Cited on p. 2, 16, 86

[15] Murnane, M. M., Kapteyn, H. C., Rosen, M. D., and Falcone, R. W. Ultrafast Xray pulses from laser-produced plasmas. Science, 251(4993):531-536, 1991. Cited on p. 2,87

[16] Kmetec, J. D., Gordon, C. L., Macklin, J. J., Lemoff, B. E., Brown, G. S., and Harris, S. E. MeV x-ray generation with a femtosecond laser. Phys. Rev. Lett., 68: 1527-1530, 1992. Cited on p. 2, 87

[17] Gibbon, P. and Förster, E. Short-pulse laser-plasma interactions. Plasma physics and controlled fusion, 38(6):769, 1996. Cited on p. 2, 87

[18] Rose-Petruck, C., Jimenez, R., Guo, T., Cavalleri, A., Siders, C. W., Rksi, F., Squier, J. A., Walker, B. C., Wilson, K. R., and Barty, C. P. Picosecondmilliångström lattice dynamics measured by ultrafast $\mathrm{X}$-ray diffraction. Nature, 398(6725):310-312, 1999. Cited on p. 2 
[19] Vogel, A., Noack, J., Hüttman, G., and Paltauf, G. Mechanisms of femtosecond laser nanosurgery of cells and tissues. Applied Physics B, 81(8):1015-1047, 2005. Cited on p. 2

[20] Herink, G., Solli, D. R., Gulde, M., and Ropers, C. Field-driven photoemission from nanostructures quenches the quiver motion. Nature, 483(7388):190-193, 2012. Cited on p. 3, 60, 63, 65, 74, 76, 81

[21] Wimmer, L., Herink, G., Solli, D. R., Yalunin, S. V., Echternkamp, K. E., and Ropers, C. Terahertz control of nanotip photoemission. Nat Phys, 10(6):432-436, 2014. Cited on p. 3, 74, 76, 77

[22] Herink, G., Wimmer, L., and Ropers, C. Field emission at terahertz frequencies: AC-tunneling and ultrafast carrier dynamics. New Journal of Physics, 16 (12):123005, 2014. Cited on p. 3

[23] Hertz, H. Ueber einen Einfluss des ultravioletten Lichtes auf die electrische Entladung. Annalen der Physik, 267(8):983-1000, 1887. Cited on p. 5

[24] Hallwachs, W. Ueber den Einfluss des Lichtes auf electrostatisch geladene Körper. Annalen der Physik, 269(2):301-312, 1888. Cited on p. 5

[25] Hallwachs, W. Ueber die Electrisirung von Metallplatten durch Bestrahlung mit electrischem Licht. Annalen der Physik, 270(8A):731-734, 1888. Cited on p. 5

[26] Lenard, P. Erzeugung von Kathodenstrahlen durch ultraviolettes Licht. Annalen der Physik, 307(6):359-375, 1900. Cited on p. 5, 7, 29

[27] Lenard, P. Ueber die lichtelektrische Wirkung. Annalen der Physik, 313(5):149_ 198, 1902. Cited on p. 5, 6, 42

[28] Millikan, R. A. A Direct Photoelectric Determination of Planck's" h". Physical Review, 7(3):355-388, 1916. Cited on p. 7

[29] Einstein, A. Über einen die Erzeugung und Verwandlung des Lichtes betreffenden heuristischen Gesichtspunkt. Annalen der Physik, 322(6):132-148, 1905. Cited on p. $6,8,42$ 
[30] Hüfner, S. Photoelectron Spectroscopy: Principles and Applications, Third Edition. Springer Berlin Heidelberg, 2003. Cited on p. 8, 29

[31] Göppert-Mayer, M. Über Elementarakte mit zwei Quantensprüngen. Annalen der Physik, 401(3):273-294, 1931. Cited on p. 8

[32] Kaiser, W. and Garrett, C. Two-Photon Excitation in Ca F 2: Eu 2+. Physical Review Letters, 7(6):229, 1961. Cited on p. 8

[33] Maiman, T. H. Stimulated optical radiation in ruby. Nature, 1960. Cited on p. 8

[34] Petite, G., Agostini, P., and Muller, H. G. Intensity dependence of non-perturbative above-threshold ionisation spectra: experimental study. Journal of Physics B: Atomic, Molecular and Optical Physics, 21(24):4097, 1988. Cited on p. 9

[35] Agostini, P., Fabre, F., Mainfray, G., Petite, G., and Rahman, N. K. Free-free transitions following six-photon ionization of xenon atoms. Physical Review Letters, 42(17):1127, 1979. Cited on p. 9

[36] Keldysh, L. V. Ionization in the field of a strong electromagnetic wave. Soviet Physics JETP, 20:1307-1314, 1965. Cited on p. 9, 10, 15, 41, 42, 83

[37] Wolkow, D. Über eine Klasse von Lösungen der Diracschen Gleichung. Zeitschrift für Physik, 94(3-4):250-260, 1935. Cited on p. 9

[38] Freeman, R. R. and Bucksbaum, P. H. Investigations of above-threshold ionization using subpicosecond laser pulses. Journal of Physics B: Atomic, Molecular and Optical Physics, 24(2):325, 1991. Cited on p. 9, 10

[39] Larochelle, S., Talebpour, A., and Chin, S.-L. Non-sequential multiple ionization of rare gas atoms in a Ti: Sapphire laser field. Journal of Physics B: Atomic, Molecular and Optical Physics, 31(6):1201, 1998. Cited on p. 10

[40] Hund, F. Zur Deutung der Molekelspektren. III. Zeitschrift für Physik, 43(11-12): 805-826, 1927. Cited on p. 10

[41] Oppenheimer, J. R. Three notes on the quantum theory of aperiodic effects. Physical review, 31(1):66, 1928. Cited on p. 10 
[42] Damon, E. and Tomlinson, R. Observation of ionization of gases by a ruby laser. Applied Optics, 2(5):546-546, 1963. Cited on p. 10

[43] Ammosov, M. V., Delone, N. B., and Krainov, V. P. Tunnel ionization of complex atoms and atomic ions in electromagnetic field. In 1986 Quebec Symposium, pages 138-141. International Society for Optics and Photonics, 1986. Cited on p. 11

[44] Fowler, R. H. and Nordheim, L. Electron Emission in Intense Electric Fields. Royal Society of London Proceedings Series A, 119:173-181, 1928. Cited on p. 11, 74

[45] Gomer, R. Field Emission and Field Ionization. Harvard University Press, Cambridge, Massachusetts, 1961. Cited on p. 11, 74, 75, 78

[46] Miller, M. K., Cerezo, A., Hetherington, M., and Smith, G. Atom probe field ion microscopy. 1996. Cited on p. 11, 35, 36

[47] Ibach, L. Festkörperphysik, 6. Auflage. Springer Berlin Heidelberg, 2002. Cited on p. 12

[48] Büttiker, M. and Landauer, R. Traversal time for tunneling. Physical Review Letters, 49(23):1739, 1982. Cited on p. 12

[49] Orlando, G., McDonald, C., Protik, N., Vampa, G., and Brabec, T. Tunnelling time, what does it mean? Journal of Physics B: Atomic, Molecular and Optical Physics, 47(20):204002, 2014. Cited on p. 12

[50] Eckle, P., Smolarski, M., Schlup, P., Biegert, J., Staudte, A., Schöffler, M., Muller, H. G., Dörner, R., and Keller, U. Attosecond angular streaking. Nature Physics, 4 (7):565-570, 2008. Cited on p. 12

[51] Charbonnier, F., Barbour, J., Garrett, L., and Dyke, W. Basic and applied studies of field emission at microwave frequencies. Proceedings of the IEEE, 51(7):9911004, 1963. Cited on p. 12, 74

[52] Fursey, G. N. Field emission in a microwave field. Journal of Vacuum Science \& Technology B, 13(2):558-565, 1995. Cited on p. 12, 74, 76

[53] Hommelhoff, P., Kealhofer, C., and Kasevich, M. A. Ultrafast electron pulses from a tungsten tip triggered by low-power femtosecond laser pulses. Physical review letters, 97(24):247402, 2006. Cited on p. 12, 43, 46 
[54] Petek, H. and Ogawa, S. Femtosecond time-resolved two-photon photoemission studies of electron dynamics in metals. Progress in surface science, 56(4):239310, 1997. Cited on p. 12, 78, 91, 94

[55] Popmintchev, T., Chen, M.-C., Popmintchev, D., Arpin, P., Brown, S., Ališauskas, S., Andriukaitis, G., Balčiunas, T., Mücke, O. D., Pugzlys, A., et al. Bright coherent ultrahigh harmonics in the $\mathrm{keV} x$-ray regime from mid-infrared femtosecond lasers. Science, 336(6086):1287-1291, 2012. Cited on p. 13

[56] Colosimo, P., Doumy, G., Blaga, C. I., Wheeler, J., Hauri, C., Catoire, F., Tate, J., Chirla, R., March, A. M., Paulus, G. G., Muller, H. G., Agostini, P., and DiMauro, L. F. Scaling strong-field interactions towards the classical limit. Nat Phys, 4(5): 386-389, 2008. Cited on p. 14, 15, 41, 42

[57] Ropers, C., Solli, D. R., Schulz, C. P., Lienau, C., and Elsaesser, T. Localized Multiphoton Emission of Femtosecond Electron Pulses from Metal Nanotips. Phys. Rev. Lett., 98(4):043907, Jan 2007. Cited on p. 16, 43, 44, 46, 60, 61, 76

[58] Barwick, B., Corder, C., Strohaber, J., Chandler-Smith, N., Uiterwaal, C., and Batelaan, H. Laser-induced ultrafast electron emission from a field emission tip. New Journal of Physics, 9(5):142, 2007. Cited on p. 16

[59] Cho, B., Ichimura, T., Shimizu, R., and Oshima, C. Quantitative evaluation of spatial coherence of the electron beam from low temperature field emitters. Physical review letters, 92(24):246103, 2004. Cited on p. 16, 89

[60] Bormann, R., Gulde, M., Weismann, A., Yalunin, S. V., and Ropers, C. TipEnhanced Strong-Field Photoemission. Phys. Rev. Lett., 105(14):147601, 2010. Cited on p. 16, 17, 41, 43, 51, 60, 65, 81

[61] Yalunin, S., Gulde, M., and Ropers, C. Strong-field photoemission from surfaces: Theoretical approaches. Phys. Rev. B., 84:195426, 2011. Cited on p. 17, 44

[62] Yanagisawa, H., Hengsberger, M., Leuenberger, D., Klöckner, M., Hafner, C., Greber, T., and Osterwalder, J. Energy Distribution Curves of Ultrafast Laser-Induced Field Emission and Their Implications for Electron Dynamics. Phys. Rev. Lett., 107:087601, 2011. Cited on p. 16, 60, 76, 81, 94 
[63] Schenk, M., Krüger, M., and Hommelhoff, P. Strong-Field Above-Threshold Photoemission from Sharp Metal Tips. Phys. Rev. Lett., 105(25):257601, 2010. Cited on p. $16,41,43,60,65,76,81$

[64] Kim, S., Jin, J., Kim, Y.-J., Park, I.-Y., Kim, Y., and Kim, S.-W. High-harmonic generation by resonant plasmon field enhancement. Nature, 453(7196):757-760, 2008. Cited on p. $16,41,43$

[65] Park, I.-Y., Kim, S., Choi, J., Lee, D.-H., Kim, Y.-J., Kling, M. F., Stockman, M. I., and Kim, S.-W. Plasmonic generation of ultrashort extreme-ultraviolet light pulses. Nature Photonics, 5(11):677-681, 2011. Cited on p. 16

[66] Sivis, M., Duwe, M., Abel, B., and Ropers, C. Nanostructure-enhanced atomic line emission. Nature, 485:E1, 2012. Cited on p. 16, 41, 43

[67] Sivis, M., Duwe, M., Abel, B., and Ropers, C. Extreme-ultraviolet light generation in plasmonic nanostructures. Nature Physics, 9(5):304-309, 2013. Cited on p. 16

[68] Sivis, M. and Ropers, C. Generation and Bistability of a Waveguide Nanoplasma Observed by Enhanced Extreme-Ultraviolet Fluorescence. Phys. Rev. Lett., 111: 085001, 2013. Cited on p. 16, 60

[69] Paulus, G., Grasbon, F., Walther, H., Villoresi, P., Nisoli, M., Stagira, S., Priori, E., and De Silvestri, S. Absolute-phase phenomena in photoionization with few-cycle laser pulses. Nature, 414(6860):182-184, 2001. Cited on p. 16

[70] Krüger, M., Schenk, M., and Hommelhoff, P. Attosecond control of electrons emitted from a nanoscale metal tip. Nature, 475(7354):78-81, 2011. Cited on p. $16,41,43,46,50,60,76,81$

[71] Piglosiewicz, B., Schmidt, S., Park, D. J., Vogelsang, J., Grosz, P., Manzoni, C., Farinello, P., Cerullo, G., and Lienau, C. Carrier-envelope phase effects on the strong-field photoemission of electrons from metallic nanostructures. Nat Photon, 8(1):37-42, 2014. Cited on p. 16, 60, 76, 81

[72] Hilbert, S., Neukirch, A., Uiterwaal, C., and Batelaan, H. Exploring temporal and rate limits of laser-induced electron emission. Journal of Physics B: Atomic, Molecular and Optical Physics, 42(14):141001, 2009. Cited on p. 17 
[73] Spielmann, C., Curley, P. F., Brabec, T., and Krausz, F. Ultrabroadband femtosecond lasers. Quantum Electronics, IEEE Journal of, 30(4):1100-1114, 1994. Cited on p. 19

[74] Corkum, P. B. and Krausz, F. Attosecond science. Nat Phys, 3(6):381-387, 2007. Cited on p. 19

[75] Ell, R., Morgner, U., Kärtner, F., Fujimoto, J. G., Ippen, E. P., Scheuer, V., Angelow, G., Tschudi, T., Lederer, M. J., Boiko, A., et al. Generation of 5-fs pulses and octave-spanning spectra directly from a Ti: sapphire laser. Optics letters, 26 (6):373-375, 2001. Cited on p. 19

[76] Strickland, D. and Mourou, G. Compression of amplified chirped optical pulses. Optics communications, 55(6):447-449, 1985. Cited on p. 19

[77] Backus, S., Peatross, J., Huang, C., Murnane, M., and Kapteyn, H. Ti: sapphire amplifier producing millijoule-level, 21-fs pulses at $1 \mathrm{kHz}$. Optics letters, 20(19): 2000-2002, 1995. Cited on p. 19

[78] Iaconis, C. and Walmsley, I. A. Spectral phase interferometry for direct electricfield reconstruction of ultrashort optical pulses. Optics letters, 23(10):792-794, 1998. Cited on p. 20, 24

[79] Boyd, R. W. Nonlinear optics. Academic press, 2003. Cited on p. 21

[80] Lauterborn, W. and Kurz, T. Coherent optics: fundamentals and applications. Springer, 2003. Cited on p. 21

[81] Maekawa, H., Tominaga, K., and Podenas, D. Generation of 55 fs-Mid-Infrared Pulses with a $3001 / \mathrm{cm}$-Spectral Width and $\mu \mathrm{J}$-Pulse Energy. Japanese journal of applied physics, 41(3B):L329, 2002. Cited on p. 22

[82] Kaindl, R. A., Wurm, M., Reimann, K., Hamm, P., Weiner, A. M., and Woerner, M. Generation, shaping, and characterization of intense femtosecond pulses tunable from 3 to $20 \mu \mathrm{m}$. JOSA B, 17(12):2086-2094, 2000. Cited on p. 24

[83] Bates, P., Chalus, O., and Biegert, J. Ultrashort pulse characterization in the midinfrared. Optics letters, 35(9):1377-1379, 2010. Cited on p. 24 
[84] Diels, J.-C., Van Stryland, E., and Benedict, G. Generation and measurement of 200 femtosecond optical pulses. Optics Communications, 25(1):93-96, 1978. Cited on p. 24

[85] Trebino, R., DeLong, K. W., Fittinghoff, D. N., Sweetser, J. N., Krumbügel, M. A., Richman, B. A., and Kane, D. J. Measuring ultrashort laser pulses in the timefrequency domain using frequency-resolved optical gating. Review of Scientific Instruments, 68(9):3277-3295, 1997. Cited on p. 24

[86] Hu, B. B. and Nuss, M. C. Imaging with terahertz waves. Opt. Lett., 20(16): 1716-1718, 1995. Cited on p. 25

[87] Beard, M. C., Turner, G. M., and Schmuttenmaer, C. A. Terahertz spectroscopy. The Journal of Physical Chemistry B, 106(29):7146-7159, 2002. Cited on p. 25

[88] Schmuttenmaer, C. A. Exploring dynamics in the far-infrared with terahertz spectroscopy. Chemical Reviews-Columbus, 104(4):1759-1780, 2004. Cited on p. 25, $59,60,73$

[89] Hoffmann, M. C. and Fülöp, J. A. Intense ultrashort terahertz pulses: generation and applications. Journal of Physics D: Applied Physics, 44(8):083001, 2011. Cited on p. 25,74

[90] Löffler, T., Kreß, M., Thomson, M., Hahn, T., Hasegawa, N., and Roskos, H. G. Comparative performance of terahertz emitters in amplifier-laser-based systems. Semiconductor Science and technology, 20(7):S134, 2005. Cited on p. 25, 26

[91] Kampfrath, T., Tanaka, K., and Nelson, K. A. Resonant and nonresonant control over matter and light by intense terahertz transients. Nature Photonics, 7(9):680690, 2013. Cited on p. 25, 59, 60, 74

[92] Leitenstorfer, A., Nelson, K. A., Reimann, K., and Tanaka, K. Focus on nonlinear terahertz studies. New Journal of Physics, 16(4):045016, 2014. Cited on p. 25, 74

[93] Auston, D. and Smith, P. Generation and detection of millimeter waves by picosecond photoconductivity. Applied Physics Letters, 43(7):631-633, 1983. Cited on p. 25 
[94] Vicario, C., Monoszlai, B., and Hauri, C. P. GV/m Single-Cycle Terahertz Fields from a Laser-Driven Large-Size Partitioned Organic Crystal. Physical Review Letters, 112(21):213901, 2014. Cited on p. 25

[95] Cook, D. J. and Hochstrasser, R. M. Intense terahertz pulses by four-wave rectification in air. Opt. Lett., 25(16):1210-1212, 2000. Cited on p. 25, 26, 60, 61, 66, 68,74

[96] Bartel, T., Gaal, P., Reimann, K., Woerner, M., and Elsaesser, T. Generation of single-cycle THz transients with high electric-field amplitudes. Opt. Lett., 30(20): 2805-2807, 2005. Cited on p. 25, 27, 61, 66, 68, 74

[97] Kim, K.-Y., Glownia, J. H., Taylor, A. J., and Rodriguez, G. Terahertz emission from ultrafast ionizing air in symmetry-broken laser fields. Optics Express, 15(8): 4577-4584, 2007. Cited on p. 26

[98] Karpowicz, N. and Zhang, X.-C. Coherent Terahertz Echo of Tunnel Ionization in Gases. Phys. Rev. Lett., 102:093001, 2009. Cited on p. 26

[99] Winnewisser, C., Jepsen, P. U., Schall, M., Schyja, V., and Helm, H. Electro-optic detection of $\mathrm{THz}$ radiation in $\mathrm{LiTaO} 3, \mathrm{LiNbO} 3$ and $\mathrm{ZnTe}$. Applied Physics Letters, 70(23):3069-3071, 1997. Cited on p. 27

[100] Ferguson, B. and Zhang, X.-C. Materials for terahertz science and technology. Nature materials, 1(1):26-33, 2002. Cited on p. 27

[101] Löffler, T. Erzeugung intensiver Pulse im Terahertzfrequenzbereich mittels lasergenerierter Plasmen. PhD thesis, Johann Wolfgang Goethe Universität, Frankfurt am Main, 2003. Cited on p. 27

[102] Boyd, R. W. Intuitive explanation of the phase anomaly of focused light beams. JOSA, 70(7):877-880, 1980. Cited on p. 29

[103] Winful, H. G. et al. Physical origin of the Gouy phase shift. Optics letters, 26(8): 485-487, 2001. Cited on p. 29

[104] Kogelnik, H. and Li, T. Laser beams and resonators. Applied Optics, 5(10):15501567, 1966. Cited on p. 29 
[105] Kaesdorf, S. Technical report, 2012. Time-of-Flight Spectrometer ETF11 for Electron Spectroscopy. Cited on p. 30

[106] Novotny, L. and Hecht, B. Principles of nano-optics. Cambridge university press, 2012. Cited on p. 33, 34

[107] Maier, S. A. Plasmonics: Fundamentals and Applications: Fundamentals and Applications. Springer, 2007. Cited on p. 34

[108] Novotny, L., Bian, R. X., and Xie, X. S. Theory of nanometric optical tweezers. Physical Review Letters, 79(4):645, 1997. Cited on p. 34, 35

[109] Bouhelier, A., Beversluis, M., Hartschuh, A., and Novotny, L. Near-Field SecondHarmonic Generation Induced by Local Field Enhancement. Phys. Rev. Lett., 90 (1):013903, 2003. Cited on p. 34, 44

[110] Neacsu, C. C., Reider, G. A., and Raschke, M. B. Second-harmonic generation from nanoscopic metal tips: Symmetry selection rules for single asymmetric nanostructures. Physical Review B, 71(20):201402, 2005. Cited on p. 34

[111] Neacsu, C., Steudle, G., and Raschke, M. Plasmonic light scattering from nanoscopic metal tips. Applied Physics B, 80(3):295-300, 2005. Cited on p. 34

[112] Thomas, S., Krüger, M., Förster, M., Schenk, M., and Hommelhoff, P. Probing of optical near-fields by electron rescattering on the $1 \mathrm{~nm}$ scale. Nano letters, 13(10): 4790-4794, 2013. Cited on p. 35

[113] Nowak, C., Kirchheim, R., and Schmitz, G. Electric-field-induced low temperature oxidation of tungsten nanowires. Applied physics letters, 89(14):143104, 2006. Cited on p. 35

[114] Johnson, P. B. and Christy, R.-W. Optical constants of the noble metals. Physical Review B, 6(12):4370, 1972. Cited on p. 36

[115] Rácz, P., Irvine, S., Lenner, M., Mitrofanov, A., Baltuška, A., Elezzabi, A., and Dombi, P. Strong-field plasmonic electron acceleration with few-cycle, phasestabilized laser pulses. Applied Physics Letters, 98(11):111116, 2011. Cited on p. $36,41,44$ 
[116] Polyanskiy, M. Refractiveindex.info, July 2014. Cited on p. 36

[117] Ibe, J., Bey Jr, P., Brandow, S., Brizzolara, R., Burnham, N., DiLella, D., Lee, K., Marrian, C., and Colton, R. On the electrochemical etching of tips for scanning tunneling microscopy. Journal of Vacuum Science \& Technology A, 8(4):35703575, 1990. Cited on p. 36

[118] Ren, B., Picardi, G., and Pettinger, B. Preparation of gold tips suitable for tipenhanced Raman spectroscopy and light emission by electrochemical etching. Review of Scientific Instruments, 75(4):837-841, 2004. Cited on p. 36

[119] Lin, Z., Zhigilei, L. V., and Celli, V. Electron-phonon coupling and electron heat capacity of metals under conditions of strong electron-phonon nonequilibrium. Physical Review B, 77(7):075133, 2008. Cited on p. 37, 78

[120] Jiang, Z. and Zhang, X.-C. Electro-optic measurement of THz field pulses with a chirped optical beam. Applied Physics Letters, 72(16):1945-1947, 1998. Cited on p. 37

[121] Bhushan, A., Coppinger, F., and Jalali, B. Time-stretched analogue-to-digital conversion. Electronics Letters, 34(11):1081-1083, 1998. Cited on p. 37

[122] Solli, D., Herink, G., Jalali, B., and Ropers, C. Fluctuations and correlations in modulation instability. Nature Photonics, 6(7):463-468, 2012. Cited on p. 37

[123] Krausz, F. and Stockman, M. I. Attosecond metrology: from electron capture to future signal processing. Nat Photon, 8(3):205-213, 2014. Cited on p. 37

[124] Demtröder. Laserspektroskopie 2. Springer Berlin / Heidelberg, 2013. Cited on p. 37

[125] Hamamatsu. Datasheet: Femtosecond Streak Camera C6138 (FESCA-200), July 2014. Cited on p. 38

[126] Quéré, F., Mairesse, Y., and Itatani, J. Temporal characterization of attosecond XUV fields. Journal of modern optics, 52(2-3):339-360, 2005. Cited on p. 38

[127] Goulielmakis, E., Uiberacker, M., Kienberger, R., Baltuska, A., Yakovlev, V., Scrinzi, A., Westerwalbesloh, T., Kleineberg, U., Heinzmann, U., Drescher, M., 
et al. Direct measurement of light waves. Science, 305(5688):1267-1269, 2004. Cited on p. 38,39

[128] Stockman, M. I., Kling, M. F., Kleineberg, U., and Krausz, F. Attosecond nanoplasmonic-field microscope. Nat Photon, 1(9):539-544, 2007. Cited on p. 39, 43,63

[129] Frühling, U., Wieland, M., Gensch, M., Gebert, T., Schutte, B., Krikunova, M., Kalms, R., Budzyn, F., Grimm, O., Rossbach, J., Plonjes, E., and Drescher, M. Single-shot terahertz-field-driven X-ray streak camera. Nat Photon, 3(9):523-528, 2009. ISSN 1749-4885. Cited on p. 39, 59, 63

[130] Schütte, B., Frühling, U., Wieland, M., Azima, A., and Drescher, M. Electron wave packet sampling with laser-generated extreme ultraviolet and terahertz fields. Opt. Express, 19(20):18833-18841, 2011. Cited on p. 39, 59

[131] Schultze, M., Fieß, M., Karpowicz, N., Gagnon, J., Korbman, M., Hofstetter, M., Neppl, S., Cavalieri, A., Komninos, Y., Mercouris, T., et al. Delay in photoemission. Science, 328(5986):1658-1662, 2010. Cited on p. 39

[132] Süßmann, F. and Kling, M. F. Attosecond nanoplasmonic streaking of localized fields near metal nanospheres. Phys. Rev. B, 84:121406, 2011. Cited on p. 39, 43, 60

[133] Skopalová, E., Lei, D. Y., Witting, T., Arrell, C., Frank, F., Sonnefraud, Y., Maier, S. A., Tisch, J. W. G., and Marangos, J. P. Numerical simulation of attosecond nanoplasmonic streaking. New Journal of Physics, 13(8):083003, 2011. Cited on p. 39,60

[134] Kelkensberg, F., Koenderink, A., and Vrakking, M. Attosecond streaking in a nano-plasmonic field. New Journal of Physics, 14(9):093034, 2012. Cited on p. 39,60

[135] Borisov, A. G., Echenique, P. M., and Kazansky, A. K. Attostreaking with metallic nano-objects. New Journal of Physics, 14(2):023036, 2012. Cited on p. 39, 60

[136] Süßmann, F. Attosecond dynamics of nano-localized fields probed by photoelectron spectroscopy. $\mathrm{PhD}$ thesis, LMU München, 2013. Cited on p. 40 
[137] Irvine, S. E., Dechant, A., and Elezzabi, A. Y. Generation of 0.4-keV Femtosecond Electron Pulses using Impulsively Excited Surface Plasmons. Phys. Rev. Lett., 93 (18):184801, 2004. Cited on p. 41, 44

[138] Zherebtsov, S., Fennel, T., Plenge, J., Antonsson, E., Znakovskaya, I., Wirth, A., Herrwerth, O., Süßmann, F., Peltz, C., Ahmad, I., Trushin, S. A., Pervak, V., Karsch, S., Vrakking, M. J. J., Langer, B., Graf, C., Stockman, M. I., Krausz, F., Ruhl, E., and Kling, M. F. Controlled near-field enhanced electron acceleration from dielectric nanospheres with intense few-cycle laser fields. Nat Phys, 7(8): 656-662, 2011. Cited on p. 41, 43, 45, 50

[139] Hentschel, M., Kienberger, R., Spielmann, C., Reider, G. A., Milosevic, N., Brabec, T., Corkum, P., Heinzmann, U., Drescher, M., and Krausz, F. Attosecond metrology. Nature, 414(6863):509-513, 2001. Cited on p. 41, 42

[140] Sansone, G., Benedetti, E., Calegari, F., Vozzi, C., Avaldi, L., Flammini, R., Poletto, L., Villoresi, P., Altucci, C., Velotta, R., Stagira, S., De Silvestri, S., and Nisoli, M. Isolated Single-Cycle Attosecond Pulses. Science, 314(5798):443-446, October 2006. Cited on p. 41, 42

[141] Gilton, T. L., Cowin, J. P., Kubiak, G. D., and Hamza, A. V. Intense surface photoemission: Space charge effects and self-acceleration. Journal of Applied Physics, 68(9):4802-4810, 1990. ISSN 00218979. Cited on p. 41, 44, 45, 87

[142] Aeschlimann, M., Schmuttenmaer, C. A., Elsayed-Ali, H. E., Miller, R. J. D., Cao, J., Gao, Y., and Mantell, D. A. Observation of surface enhanced multiphoton photoemission from metal surfaces in the short pulse limit. Journal of Chemical Physics, 102(21):8606-8613, 1995. Cited on p. 41, 44, 45, 87

[143] Petite, G., Agostini, P., Trainham, R., Mevel, E., and Martin, P. Origin of the highenergy electron emission from metals under laser irradiation. Phys. Rev. B, 45: 12210-12217, 1992. Cited on p. 41, 44, 45, 87

[144] Passlack, S., Mathias, S., Andreyev, O., Mittnacht, D., Aeschlimann, M., and Bauer, M. Space charge effects in photoemission with a low repetition, high intensity femtosecond laser source. Journal of applied physics, 100(2):024912, 2006. Cited on p. $41,44,45,87$ 
[145] Meckel, M., Comtois, D., Zeidler, D., Staudte, A., Pavičić, D., Bandulet, H., Pépin, H., Kieffer, J., Dörner, R., Villeneuve, D., et al. Laser-induced electron tunneling and diffraction. Science, 320(5882):1478-1482, 2008. Cited on p. 42

[146] Hommelhoff, P., Sortais, Y., Aghajani-Talesh, A., and Kasevich, M. A. Field Emission Tip as a Nanometer Source of Free Electron Femtosecond Pulses. Phys. Rev. Lett., 96(7):077401, 2006. Cited on p. 43, 60, 76

[147] Huber, A. J., Keilmann, F., Wittborn, J., Aizpurua, J., and Hillenbrand, R. Terahertz Near-Field Nanoscopy of Mobile Carriers in Single Semiconductor Nanodevices. Nano Letters, 8(11):3766-3770, 2008. Cited on p. 44, 73, 85

[148] Gaal, P., Kuehn, W., Reimann, K., Woerner, M., Elsaesser, T., and Hey, R. Internal motions of a quasiparticle governing its ultrafast nonlinear response. Nature, 450 (7173):1210-1213, 2007. Cited on p. 59, 60

[149] Evenson, K., Inguscio, M., and Jennings, D. Point contact diode at laser frequencies. Journal of Applied Physics, 57(3):956-960, 1985. Cited on p. 59

[150] Schiffrin, A., Paasch-Colberg, T., Karpowicz, N., Apalkov, V., Gerster, D., Muhlbrandt, S., Korbman, M., Reichert, J., Schultze, M., Holzner, S., Barth, J. V., Kienberger, R., Ernstorfer, R., Yakovlev, V. S., Stockman, M. I., and Krausz, F. Optical-field-induced current in dielectrics. Nature, 493(7430):70-74, 2013. Cited on p. 60

[151] Fleischer, S., Zhou, Y., Field, R. W., and Nelson, K. A. Molecular Orientation and Alignment by Intense Single-Cycle THz Pulses. Phys. Rev. Lett., 107:163603, 2011. Cited on p. 60

[152] Liu, M., Hwang, H. Y., Tao, H., Strikwerda, A. C., Fan, K., Keiser, G. R., Sternbach, A. J., West, K. G., Kittiwatanakul, S., Lu, J., et al. Terahertz-field-induced insulator-to-metal transition in vanadium dioxide metamaterial. Nature, 487(7407): 345-348, 2012. Cited on p. 60

[153] Ciappina, M., Pérez-Hernández, J., Shaaran, T., Biegert, J., Quidant, R., and Lewenstein, M. Above-threshold ionization by few-cycle spatially inhomogeneous fields. Physical Review A, 86(2):023413, 2012. Cited on p. 60 
[154] Yalunin, S. V., Herink, G., Solli, D. R., Krüger, M., Hommelhoff, P., Diehn, M., Munk, A., and Ropers, C. Field localization and rescattering in tip-enhanced photoemission. Annalen der Physik, 525(1-2):L12-L18, 2013. Cited on p. 60

[155] Park, D. J., Piglosiewicz, B., Schmidt, S., Kollmann, H., Mascheck, M., and Lienau, C. Strong Field Acceleration and Steering of Ultrafast Electron Pulses from a Sharp Metallic Nanotip. Physical review letters, 109(24):244803, 2012. Cited on p. 60, 76

[156] Kim, K. T., Zhang, C., Ruchon, T., Hergott, J.-F., Auguste, T., M., V., B., C., and QuereF. Photonic streaking of attosecond pulse trains. Nat Photon, 7(8):651-656, 2013. Cited on p. 60

[157] Kirchner, F., Gliserin, A., Krausz, F., and Baum, P. Laser streaking of free electrons at $25 \mathrm{keV}$. Nature Photonics, 2013. Cited on p. 60

[158] Schubert, O., Riek, C., Junginger, F., Sell, A., Leitenstorfer, A., and Huber, R. Ultrashort pulse characterization with a terahertz streak camera. Opt. Lett., 36(22): 4458-4460, 2011. Cited on p. 61

[159] Wang, K., Mittleman, D. M., van der Valk, N. C. J., and Planken, P. C. M. Antenna effects in terahertz apertureless near-field optical microscopy. Applied Physics Letters, 85(14):2715-2717, 2004. Cited on p. 61, 70, 75

[160] Kersting, R., Chen, H.-T., Karpowicz, N., and Cho, G. C. Terahertz microscopy with submicrometre resolution. Journal of Optics A: Pure and Applied Optics, 7 (2):S184, 2005. Cited on p. 61, 70

[161] Grguras., R., M. A., C., B., T., M., J., K. T., P., R., S., D., K., K. A., M., K. N., Th., T., T., C. J., M., M., C., H. M., H., S., and L., C. A. Ultrafast X-ray pulse characterization at free-electron lasers. Nat Photon, 6(12):852-857, 2012. Cited on p. 63

[162] van Oudheusden, T., Pasmans, P. L. E. M., van der Geer, S. B., de Loos, M. J., van der Wiel, M. J., and Luiten, O. J. Compression of Subrelativistic Space-Charge-Dominated Electron Bunches for Single-Shot Femtosecond Electron Diffraction. Phys. Rev. Lett., 105:264801, 2010. Cited on p. 65, 87 
[163] Ulbricht, R., Hendry, E., Shan, J., Heinz, T. F., and Bonn, M. Carrier dynamics in semiconductors studied with time-resolved terahertz spectroscopy. Reviews of Modern Physics, 83(2):543, 2011. Cited on p. 66

[164] Cocker, T. L., Jelic, V., Gupta, M., Molesky, S. J., J., B. A., Reyes, G. D. L., Titova, L. V., Tsui, Y. Y., Freeman, M. R., and Hegmann, F. A. An ultrafast terahertz scanning tunnelling microscope. Nature Photonics, 7(8):620-625, 2013. ISSN 1749-4885. Cited on p. 67, 74

[165] Tonouchi, M. Cutting-edge terahertz technology. Nature Photonics, 1(2):97-105, 2007. Cited on p. 73

[166] Mittleman, D. M. Frontiers in terahertz sources and plasmonics. Nature Photonics, 7(9):666-669, 2013. Cited on p. 74

[167] Greig, S. R. and Elezzabi, A. Y. On the role of terahertz field acceleration and beaming of surface plasmon generated ultrashort electron pulses. Applied Physics Letters, 105(4):041115, 2014. Cited on p. 74

[168] Ronny Huang, W., Nanni, E. A., Ravi, K., Hong, K.-H., Jie Wong, L., Keathley, P. D., Fallahi, A., Zapata, L., and Kärtner, F. X. A terahertz-driven electron gun. ArXiv e-prints, September 2014. Cited on p. 74, 87

[169] Kuehn, W., Gaal, P., Reimann, K., Woerner, M., Elsaesser, T., and Hey, R. Terahertz-induced interband tunneling of electrons in GaAs. Physical Review B, 82(7):075204, 2010. Cited on p. 74

[170] Somma, C., Reimann, K., Flytzanis, C., Elsaesser, T., and Woerner, M. HighField Terahertz Bulk Photovoltaic Effect in Lithium Niobate. Phys. Rev. Lett., 112: 146602, 2014. Cited on p. 74

[171] Hommelhoff, P., Kealhofer, C., and Kasevich, M. Reaching the resolved tunnel regime for a femtosecond oscillator driven field emission electron source. Laser Physics, 19:736-738, 2009. Cited on p. 74

[172] Zhong, H., Karpowicz, N., and Zhang, X.-C. Terahertz emission profile from laserinduced air plasma. Applied physics letters, 88(26):261103, 2006. Cited on p. 75 
[173] You, Y., Oh, T., and Kim, K. Off-axis phase-matched terahertz emission from twocolor laser-induced plasma filaments. Physical review letters, 109(18):183902, 2012. Cited on p. 75

[174] Klarskov, P., Strikwerda, A. C., Iwaszczuk, K., and Jepsen, P. U. Experimental three-dimensional beam profiling and modeling of a terahertz beam generated from a two-color air plasma. New Journal of Physics, 15(7):075012, 2013. Cited on p. 75

[175] Blank, V., Thomson, M., and Roskos, H. Spatio-spectral characteristics of ultrabroadband THz emission from two-colour photoexcited gas plasmas and their impact for nonlinear spectroscopy. New Journal of Physics, 15(7):075023, 2013. Cited on p. 75

[176] Dombi, P., Hörl, A., Rácz, P., Márton, I., Trügler, A., Krenn, J. R., and Hohenester, U. Ultrafast Strong-Field Photoemission from Plasmonic Nanoparticles. Nano Letters, 13(2):674-678, 2013. Cited on p. 76

[177] Behr, N. and Raschke, M. B. Optical antenna properties of scanning probe tips: plasmonic light scattering, tip-sample coupling, and near-field enhancement. The Journal of Physical Chemistry C, 112(10):3766-3773, 2008. Cited on p. 76

[178] Ropers, C., Elsaesser, T., Cerullo, G., Zavelani-Rossi, M., and Lienau, C. Ultrafast optical excitations of metallic nanostructures: from light confinement to a novel electron source. New Journal of Physics, 9(10):397, 2007. Cited on p. 76

[179] Yanagisawa, H., Hafner, C., Doná, P., Klöckner, M., Leuenberger, D., Greber, T., Osterwalder, J., and Hengsberger, M. Laser-induced field emission from a tungsten tip: Optical control of emission sites and the emission process. Phys. Rev. B, 81: 115429, 2010. Cited on p. 76

[180] Brorson, S., Fujimoto, J., and Ippen, E. Femtosecond electronic heat-transport dynamics in thin gold films. Physical Review Letters, 59:1962-1965, 1987. Cited on p. $78,80,91,96$

[181] Hohlfeld, J., Wellershoff, S.-S., Güdde, J., Conrad, U., Jähnke, V., and Matthias, E. Electron and lattice dynamics following optical excitation of metals. Chemical Physics, 251(1):237-258, 2000. Cited on p. 78, 80, 91, 95, 96 
[182] Voisin, C., Del Fatti, N., Christofilos, D., and Vallée, F. Ultrafast Electron Dynamics and Optical Nonlinearities in Metal Nanoparticles. The Journal of Physical Chemistry B, 105(12):2264-2280, 2001. Cited on p. 78

[183] Wu, L. and Ang, L. K. Nonequilibrium model of ultrafast laser-induced electron photofield emission from a dc-biased metallic surface. Phys. Rev. B, 78:224112, 2008. Cited on p. 78

[184] Anisimov, S., Kapeliovich, B., and Perel'Man, T. Electron emission from metal surfaces exposed to ultrashort laser pulses. Zh. Eksp. Teor. Fiz, 66(776):375-7, 1974. Cited on p. 78,95

[185] Hodak, J. H., Henglein, A., and Hartland, G. V. Photophysics of Nanometer Sized Metal Particles: Electron-Phonon Coupling and Coherent Excitation of Breathing Vibrational Modes. The Journal of Physical Chemistry B, 104(43):9954-9965, 2000. Cited on p. 80

[186] Fujimoto, J. G., Liu, J. M., Ippen, E. P., and Bloembergen, N. Femtosecond Laser Interaction with Metallic Tungsten and Nonequilibrium Electron and Lattice Temperatures. Phys. Rev. Lett., 53:1837-1840, 1984. Cited on p. 80

[187] Daraszewicz, S. L., Giret, Y., Tanimura, H., Duffy, D. M., Shluger, A. L., and Tanimura, K. Determination of the electron-phonon coupling constant in tungsten. Applied Physics Letters, 105(2):023112, 2014. Cited on p. 80

[188] Kealhofer, C., Foreman, S. M., Gerlich, S., and Kasevich, M. A. Ultrafast lasertriggered emission from hafnium carbide tips. Physical Review B, 86(3):035405, 2012. Cited on p. 81

[189] Ropers, C., Neacsu, C., Elsaesser, T., Albrecht, M., Raschke, M., and Lienau, C. Grating-coupling of surface plasmons onto metallic tips: a nanoconfined light source. Nano letters, 7(9):2784-2788, 2007. Cited on p. 85

[190] Gramotnev, D. K. and Bozhevolnyi, S. I. Nanofocusing of electromagnetic radiation. Nature Photonics, 8(1):13-22, 2014. Cited on p. 85

[191] Stockman, M. I. Nanofocusing of optical energy in tapered plasmonic waveguides. Physical review letters, 93(13):137404, 2004. Cited on p. 85 
[192] Mühlschlegel, P., Eisler, H.-J., Martin, O., Hecht, B., and Pohl, D. Resonant optical antennas. Science, 308(5728):1607-1609, 2005. Cited on p. 85

[193] Linden, S., Enkrich, C., Wegener, M., Zhou, J., Koschny, T., and Soukoulis, C. M. Magnetic response of metamaterials at 100 terahertz. Science, 306(5700):13511353, 2004. Cited on p. 85

[194] Müller, M., Paarmann, A., and Ernstorfer, R. Femtosecond electrons probing currents and atomic structure in nanomaterials. arXiv preprint arXiv:1405.4992, 2014. Cited on p. 87

[195] Hebeisen, C. T., Ernstorfer, R., Harb, M., Dartigalongue, T., Jordan, R. E., and Dwayne Miller, R. Femtosecond electron pulse characterization using laser ponderomotive scattering. Optics letters, 31(23):3517-3519, 2006. Cited on p. 87

[196] Park, H. and Zuo, J. Direct measurement of transient electric fields induced by ultrafast pulsed laser irradiation of silicon. Applied Physics Letters, 94(25):251103, 2009. Cited on p. 87

[197] Raman, R. K., Tao, Z., Han, T.-R., and Ruan, C.-Y. Ultrafast imaging of photoelectron packets generated from graphite surface. Applied Physics Letters, 95(18): 181108, 2009. Cited on p. 87

[198] Barwick, B., Flannigan, D. J., and Zewail, A. H. Photon-induced near-field electron microscopy. Nature, 462(7275):902-906, 2009. Cited on p. 87

[199] Park, S. T., Lin, M., and Zewail, A. H. Photon-induced near-field electron microscopy (PINEM): theoretical and experimental. New Journal of Physics, 12(12): 123028, 2010. Cited on p. 87

[200] Foreman, S. M., Kealhofer, C., Skulason, G. E., Klopfer, B. B., and Kasevich, M. A. Ultrafast microfocus x-ray source based on a femtosecond laser-triggered tip. Annalen der Physik, 525(1-2):L19-L22, 2013. Cited on p. 87

[201] Zamponi, F., Ansari, Z., Wörner, M., and Elsässer, T. Femtosecond powder diffraction with a laser-driven hard X-ray source. Optics express, 18(2):947-961, 2010. Cited on p. 87 
[202] Reusch, T. Non-equilibrium dynamics of lipid bilayers Time resolved $x$-ray scattering at in-house and synchrotron sources. $\mathrm{PhD}$ thesis, Georg-August-Universität Göttingen, 2013. Cited on p. 87

[203] Gordon, C. L., Yin, G., Lemoff, B., Bell, P. M., and Barty, C. Time-gated imaging with an ultrashort-pulse, laser-produced-plasma x-ray source. Optics letters, 20(9): 1056-1058, 1995. Cited on p. 87

[204] Tsujino, S., Beaud, P., Kirk, E., Vogel, T., Sehr, H., Gobrecht, J., and Wrulich, A. Ultrafast electron emission from metallic nanotip arrays induced by near infrared femtosecond laser pulses. Applied Physics Letters, 92(19):193501, 2008. Cited on p. 87

[205] Patterson, B., Abela, R., Braun, H., Flechsig, U., Ganter, R., Kim, Y., Kirk, E., Oppelt, A., Pedrozzi, M., Reiche, S., et al. Coherent science at the SwissFEL X-ray laser. New Journal of Physics, 12(3):035012, 2010. Cited on p. 87

[206] Swanwick, M. E., Keathley, P. D., Fallahi, A., Krogen, P. R., Laurent, G., Moses, J., Kärtner, F. X., and Velásquez-García, L. F. Nanostructured Ultrafast Silicon-Tip Optical Field-Emitter Arrays. Nano letters, 14(9):5035-5043, 2014. Cited on p. 87

[207] Ioakeimidi, K., Leheny, R. F., Gradinaru, S., Bolton, P. R., Aldana, R., Ma, K., Clendenin, J. E., Harris, J. S., and Pease, R. F. W. Photoelectronic analog-todigital conversion: sampling and quantizing at $100 \mathrm{Gs} / \mathrm{s}$. Microwave Theory and Techniques, IEEE Transactions on, 53(1):336-342, 2005. Cited on p. 89

[208] Fabiańska, J., Kassier, G., and Feurer, T. Split ring resonator based THz-driven electron streak camera featuring femtosecond resolution. Scientific reports, 4, 2014. Cited on p. 90

[209] Utsumi, T. Vacuum microelectronics: What's new and exciting. Electron Devices, IEEE Transactions on, 38(10):2276-2283, 1991. Cited on p. 90

[210] Spindt, C., Holland, C., Rosengreen, A., and Brodie, I. Field-emitter arrays for vacuum microelectronics. Electron Devices, IEEE Transactions on, 38(10):23552363, 1991. Cited on p. 90 
[211] Savage, K. J., Hawkeye, M. M., Esteban, R., Borisov, A. G., Aizpurua, J., and Baumberg, J. J. Revealing the quantum regime in tunnelling plasmonics. Nature, 491(7425):574-577, 2012. Cited on p. 90

[212] Miller, D. A. Are optical transistors the logical next step? Nature Photonics, 4(1): 3-5, 2010. Cited on p. 90

[213] Gramotnev, D. K. and Bozhevolnyi, S. I. Plasmonics beyond the diffraction limit. Nature Photonics, 4(2):83-91, 2010. Cited on p. 90

[214] Rewitz, C., Razinskas, G., Geisler, P., Krauss, E., Goetz, S., Pawłowska, M., Hecht, B., and Brixner, T. Coherent Control of Plasmon Propagation in a Nanocircuit. Physical Review Applied, 1(1):014007, 2014. Cited on p. 90

[215] Schnell, M., Alonso-Gonzalez, P., Arzubiaga, L., Casanova, F., Hueso, L., Chuvilin, A., and Hillenbrand, R. Nanofocusing of mid-infrared energy with tapered transmission lines. Nature photonics, 5(5):283-287, 2011. Cited on p. 90

[216] Stanley, R. Plasmonics in the mid-infrared. Nature Photonics, 6(7):409-411, 2012. Cited on p. 90

[217] Houard, J., Vella, A., Vurpillot, F., and Deconihout, B. Three-dimensional thermal response of a metal subwavelength tip under femtosecond laser illumination. Physical Review B, 84(3):033405, 2011. Cited on p. 91

[218] Müller, E. W. Das Feldionenmikroskop. Zeitschrift für Physik, 131(1):136-142, 1951. Cited on p. 91

[219] Faulhaber, A., Smith, B., Andersen, J., and Zhang, J. Femtosecond electronic relaxation dynamics in metal nano-particles: Effects of surface and size confinement. Molecular Crystals and Liquid Crystals, 283(1):25-30, 1996. Cited on p. 96

[220] Muskens, O. L., Del Fatti, N., and Vallée, F. Femtosecond response of a single metal nanoparticle. Nano letters, 6(3):552-556, 2006. Cited on p. 96 


\section{Publications and conference talks}

\section{Publications}

The following articles contribute to the cumulative thesis.

- Herink, G., Wimmer, L., and Ropers, C., "Field emission at Terahertz frequencies: AC-tunneling and ultrafast carrier dynamics in nanotips", New Journal of Physics, 16, 123005, (2014)

- Wimmer, L.*, Herink, G.*, Solli, D. R., Yalunin, S. V., Echternkamp, K.E., and Ropers, C. "Terahertz control of nanotip photoemission", Nature Physics, 10, 432436 (2014). (* Equal contribution)

- Herink, G., Solli, D. R., Gulde, M., and Ropers, C., "Field-driven photoemission from nanostructures quenches the quiver motion", Nature, 483, 190-193 (2012).

The following articles originated over the course of this work, and are not a direct part of the cumulative thesis.

- Herink, G., Solli, D. R., Gulde, M., and Ropers, C., "Strong field photoemission from metal nanotips", in Sakabe, S., Lienau, C., Grunwald, R. (Eds.), Progress in Nonlinear Nanooptics, Springer Verlag, Heidelberg, in press.

- Herink, G, Solli, D. R., Gulde, M., and Ropers, C., "Photoeffekt an Nanostrukturen: der klassische Grenzfall.", Physik in unserer Zeit, 43, 165-166 (2012).

- Solli, D. R., Herink, G., Jalali, B., and Ropers, C., "Fluctuations and correlations in modulation instability", Nature Photonics, 6, 463-468 (2012).

- Yalunin, S. V., Herink, G., Solli, D. R., Krüger, M., Hommelhoff, P., Diehn, M., Munk, A., and Ropers, C., "Field localization and rescattering in tip-enhanced photoemission", Annalen der Physik, 525, L12-L18 (2012). 


\section{Conference talks}

- "Enhanced THz-near-field controls nanotip photoemission." Key-note talk, International Society of Infrared, Millimeter, and Terahertz Waves: IRMMW-THz 2014, Tuscon, USA.

- "Field-driven THz-streaking of Nanotip Photoemission", DPG Spring Meeting 2014, Berlin.

- "Controlling ultrafast photoelectron dynamics in nanostructure-enhanced THz fields", Optical Society of America, High Intensity Lasers and High Field Phenomena, HILAS 2014, Berlin, Germany.

- "Ultrafast field-driven photoemission spectroscopy and control at metallic nanotips", SPIE Optics+Photonics 2013, San Diego, USA.

- "Photoemission at metallic nanostructures: multiphoton and strong-field dynamics",

SPIE Photonics West 2013, San Francisco, USA.

- "Single-shot spectroscopy of optical modulation instability", European Optical Society, EOSAM 2012, Aberdeen, Scotland (GB).

- "Mid-infrared Photoelectron Emission and Acceleration at Metallic Nanotips", Optical Society of America, High Intensity Lasers and High Field Phenomena, HILAS 2012, Berlin, Germany. 


\section{Danksagung}

Ein besonderer Dank gilt Prof. Dr. Claus Ropers für die erstklassige Betreuung der Arbeit. Sein persönliches Engagement, seine Begeisterungsfähigkeit und die inspirierenden physikalischen Diskussionen trugen wesentlich zum Gelingen der Arbeit bei und stellten eine große fachliche wie persönliche Bereicherung dar.

Prof. Dr. Tim Saditt für die freundliche Übernahme des Korreferats und interessante Einblicke in die Röntgenphysik. Ebenso danke ich Prof. Dr. Bert Hecht für sein Interesse an der Arbeit und die Übernahme des zweiten Korreferats.

Der Arbeitsgruppe danke ich für die sehr gute Atmospäre und für die Unterstützung bei der Umsetzung der Arbeit - ein großer Dank an alle derzeitigen und ehemaligen Mitstreiter! Insbesondere möchte ich mich für die herzliche Aufnahme in die Gruppe bei Dr. Murat Sivis, Reiner Bormann und Max Gulde bedanken, und für ihre tatkräftige Unterstützung insbesondere beim Start in die Laborarbeit.

I'd like to thank Dr. Daniel Solli for many exciting discussions, some really long lasertweaking evenings, and for his careful and rapid proof-reading.

Der Büro- und Laborgemeinschaft von Katharina Echternkamp, Armin Feist, Lara Wimmer und schließlich Frederik Busse für das klasse Arbeitsklima, die Flora und das Erdulden etlicher Ohrwürmer und leerer Kaffeetassen. Insbesondere danke ich Lara und Katharina für die gute Zusammenarbeit.

Prof. Dr. Sergey Yalunin for many enlightening discussions on physical pictures, interpretations and ideas.

Armin Feist, Max Gulde, Benjamin Schröder und Gero Storeck für den spitze(n) Support.

Dank an Bernhard Spiecher für die kompetente technische Unterstützung (Diskussionen waren immer lohnenswert), Andreas Juretzko aus der feinmechanischen Werkstatt und den Mitarbeitern der Zentralwerkstatt für etliche, immer eilig herzustellende Bauteile.

Dank an Antje Spliethoff-Laiser, Anita Jähnke und Kerstin Reinecke für die jahrelange bürokratische Unterstützung, insbesondere bei den Bestellungen und Dienstreise- 
Formalitäten.

Ich bedanke mich bei der DFG und den Organisatoren des DFG Schwerpunktprojekts SPP-1391 "Ultrafast Nanooptics" für die finanziellen Mittel und für die gelungenen Veranstaltungen, die eine fachliche, soziale und geographische Bereicherung darstellten.

Für die schöne Zeit in Göttingen nach der Rückkehr aus Hannover danke ich besonders Dr. Benjamin Lenk, seiner lieben WG, Björn Enders, Dr. Max Neudecker, Dr. Murat Sivis, Dr. Marvin Walter und der weitverstreuten Erstsemester-Gruppe!

Ich danke meinen Eltern, meinen beiden Brüdern und den lieben Verwandten, die mich immer unterstützt haben, und allen Freunden für ein Leben außerhalb des Instituts.

Meiner Freundin Stephanie danke ich von Herzen für ihre Geduld in mancher Spätschicht, ihre Unterstützung und die Leidenschaft für ihren Physiker. 